
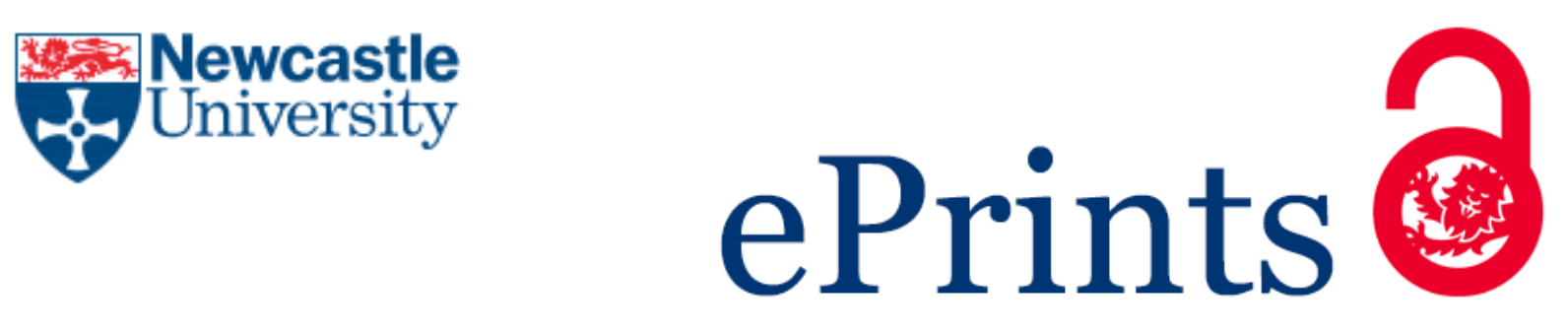
Hoegberg LC, Bania TC, Lavergne V, Bailey B, Turgeon AF, Thomas SH, Morris M, Miller-Nesbitt A, Mégarbane B, Magder S, Gosselin S.
Systematic review of the effect of intravenous lipid emulsion therapy for
local anesthetic toxicity.
Clinical Toxicology 2016, 54(3), 167-193

\title{
Copyright:
}

This is an Accepted Manuscript of an article published by Taylor \& Francis in Clinical Toxicology on 1/3/2016, available online: http://www.tandfonline.com/10.3109/15563650.2015.1121270

DOI link to article:

http://dx.doi.org/10.3109/15563650.2015.1121270

Date deposited:

$08 / 06 / 2016$

Embargo release date:

01 March 2017

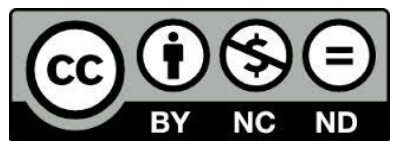

This work is licensed under a

Creative Commons Attribution-NonCommercial-NoDerivatives 4.0 International licence 
AACT manuscript LA to edit for CTX

\section{Systematic review of the effect of intravenous lipid emulsion therapy for local anesthetic} toxicity

\section{AUTHORS \& AFFILIATIONS}

1. Lotte C. G. Hoegberg, Pharmacist, PhD. Danish Poisons Information Centre, Anesthesiology, Copenhagen University Hospital, Bispebjerg, Copenhagen, Denmark

2. Theodore C. Bania, MD. Mt Sinai Roosevelt, Mt Sinai St. Luke's, Department of Emergency Medicine, Icahn School of Medicine, New York, NY, USA

3. Valéry Lavergne, MD, MSc. Department of Medical Biology, Sacré-Coeur Hospital, University Of Montréal, Montréal, Québec, Canada

4. Benoit Bailey, MD, MSc. Division of Emergency Medicine Department of Pediatrics, CHU Sainte-Justine, Montréal, Québec Canada, Centre Antipoison du Québec, Quebec, Canada

5. Alexis F. Turgeon, MD, MSc. Division of Critical Care Medicine, Department of Anesthesiology and Critical Care Medicine, and CHU de Québec - Université Laval Research Center, Population Health and Optimal Health Practices Unit, Université Laval, Québec City, Québec, Canada

6. Simon H.L. Thomas, MD. National Poisons Information Service (Newcastle) and Medical Toxicology Centre, Institute of Cellular Medicine, Newcastle University, Newcastle, UK

7. Martin Morris. Schulich Library of Science and Engineering, McGill University, Montréal, Québec, Canada

8. Andrea Nesbitt Miller. Schulich Library of Science and Engineering, McGill University, Montréal, Québec, Canada

9. Bruno Mégarbane, MD, PhD. Department of medical and toxicological intensive care, Lariboisière Hospital, INSERM U1144, Paris-Diderot University, Paris, France

10. Sheldon Magder, MD. Department of Critical Care, McGill University Health Centre, Montreal, Québec, Canada

11. Sophie Gosselin, MD. Department of Emergency Medicine, Medical Toxicology Consultation Service, McGill University Health Centre, and Centre Antipoison du Québec, Montréal, Québec, Canada

The other members of the AACT lipid emulsion workgroup are: Ashish Bhalla, Diane Calello, Ryan Chuang, Brian Gilfix, Andis Graudins, Ami Grunbaum, Bryan D. Hayes, Robert S. Hoffman, Michael Levine, Jose Morais, Carol Rollins, Samuel Stellpflug, Christine Stork 
AACT manuscript LA to edit for CTX

Corresponding author

Lotte C. G. Hoegberg, Pharmacist, PhD.

Danish Poisons Information Centre

Dept. of Anaesthesiology, Copenhagen University Hospital, Bispebjerg

Copenhagen, Denmark

Lotte.Hoegberg@ regionh.dk

$+4535315403$

WORDS:

Abstract: 405

Text: 4,257

Number of figures: 1

Number of tables: 4

Number of references: 131 


\section{AACT manuscript LA to edit for CTX}

\section{Abstract}

Background: Following national and regional recommendations, intravenous lipid emulsion (ILE) has become established in clinical practice as a treatment for acute local anesthetic (LA) toxicity, although evidence of efficacy is limited to animal studies and human case reports. A lipid emulsion workgroup was therefore established by the American Academy of Clinical Toxicology to review the evidence on the efficacy of ILE for LA toxicity.

Methods: We performed a systematic review of the literature published through December 15th 2014. Relevant articles were determined based on predefined inclusion and exclusion criteria. Pre-treatment experiments, pharmacokinetic studies not involving toxicity and studies that did not address antidotal use of ILE were excluded.

Results: We included 113 studies and reports. Of these, 75 were human and 38 animal studies. One publication included both a human case report and an animal study. Human studies included one randomized controlled crossover trial involving 16 healthy volunteers. The subclinical LA toxicity design did not show a difference in effects of ILE versus saline. There was one case series and 73 case reports of ILE use in the context of toxicity (83 patients) including CNS depression or agitation $(n=45,54 \%)$, seizures $(n=49,59 \%)$, hypotension, hypertension, $E K G$ changes, arrhythmias $(n=39,47 \%)$, cardiac arrest $(n=18,22 \%)$, cardiopulmonary resuscitation and/or requirement for endotracheal intubation and/or mechanical ventilation $(n=35,42 \%)$. There were $81(98 \%)$ survivors including $63(76 \%)$ with no reported sequelae from the LA poisoning or ILE, although the presence or absence of sequelae was not reported in 15 (18\%) cases. 


\section{AACT manuscript LA to edit for CTX}

Animal studies included 29 randomized controlled studies, 3 observational studies, 5 case series and one case report; bupivacaine was used in 29 of these reports (76\%). Of 14 controlled experiments in animals, 8 showed improved survival or time to return of spontaneous circulation and 5 no benefit of ILE versus saline or non-ILE treatments. Combining ILE with epinephrine improved survival in 5 of the 6 controlled animal experiments that studied this intervention. The studies were heterogeneous in the formulations and doses of ILE used as well as the doses of LA. The body of the literature identified by this systematic review yielded only a very low quality of evidence.

Conclusion: ILE appears to be effective for reversal of cardiovascular or neurological features in some cases of LA toxicity, but there is currently no convincing evidence showing that ILE is more effective than vasopressors or to indicate which treatment should be instituted as first line therapy in severe LA toxicity. 


\section{AACT manuscript LA to edit for CTX}

\section{Introduction}

There has been increasing interest in the use of intravenous lipid emulsion (ILE) for the treatment of acute local anesthetic (LA) poisoning following the publication of a case report in 2006.(1) Since then, national and regional anesthesiology societies have published recommendations for use of ILE in the treatment of LA toxicity after iatrogenic overdose.(2-4) However, evidence supporting the use of ILE in the context of toxicity involving local anesthetics or other toxins has been reported by previous reviews to consist primarily of human case reports and controlled animal experiments that cannot necessarily be extrapolated to human clinical settings.(5-8)

The American Academy of Clinical Toxicology (AACT) therefore created a Lipid Emulsion workgroup, which included clinical experts in clinical toxicology, anesthesiology, emergency medicine, critical care, and pharmacy with assistance of medical librarians and epidemiologists. This workgroup was tasked to review all appropriate evidence pertaining to the use of lipid emulsion in toxicology, with the ultimate goal of providing a comprehensive evaluation of the published evidence and consensus-based recommendations.(9) Here we present the results of our systematic review of human and animal studies regarding the effect of ILE in the treatment of LA toxicity. Use for treating toxicity from other substances and adverse effects of ILE will be presented in other systematic reviews. 


\section{AACT manuscript LA to edit for CTX}

\section{Methods}

A working subgroup (the authors) of the American Academy of Clinical Toxicology lipid emulsion therapy workgroup (9) was formed to gather and review the evidence on the effect of ILE in the treatment of LA toxicity. This subgroup was formed based on the best possible match to represent the clinical experts and various stakeholders and involved in the workgroup. It also included two medical librarians who assisted in conducting the systematic searches and the retrieval of potentially eligible publications, as well as an epidemiologist with specific methodological expertise in conducting systematic reviews. Subgroup members divulged all potential conflicts of interests prior to inclusion in the workgroup. All communication was performed by email exchanges and by telephone conferences.

Two medical librarians created a systematic search strategy for Medline (Ovid), which is provided at Appendix 1. The strategy comprised a combination of Medical Subject Headings, title/abstract key words, truncations, and Boolean operators, and included the concepts of ILE and toxicology (including but not limited to local anesthetics). It was subsequently translated for Embase (via Ovid), CINAHL (via EBSCO), BIOSIS Previews (via Ovid), Web of Science, Scopus, and the Cochrane Library/DARE. All databases were searched from inception to December $15^{\text {th }} 2014$. 


\section{AACT manuscript LA to edit for CTX}

In addition, conference abstracts from the European Association for Poison Centres and Clinical Toxicologists, and the North American Congress of Clinical Toxicology (both from 2000 to 2014) and previous reviews were hand-searched by various group members. Abstracts from the Asia Pacific Association of Medical Toxicology were searched in the same way from 2007 to 2014. Group members also performed cross-referencing of full-text articles. No limits were applied for language, and candidate studies in languages not known to any of the authors were translated.

In summary, the criteria for publication inclusion in the evaluation of the effect of ILE include studies in humans and animals to whom ILE was given for the purpose of treating poisoning, and exclusion criteria are non-original data, animal studies with methods and results that cannot be extrapolated or are uninterpretable to humans, pre-treatment models, and experimental in vitro or ex vivo models. A complete methodology of the larger project of which this systematic review is one part has been previously published, and describes in detail all relevant methodological aspects such as clinical questions, search strategies, eligibility of publications, data extraction and summary, and assessment of the risk of bias (9).

The $\log \mathrm{D}$, which is based on the partition coefficient, and is a measure of lipophilicity, is reported for each local anesthetic. The degree of lipophilicity directly corresponds with the $\log \mathrm{D}$; as the $\log \mathrm{D}$ increases, so does the lipophilicity of a substance. 


\section{AACT manuscript LA to edit for CTX}

\section{Results}

Our combined search for the effect of ILE retrieved 838 full text articles that were subsequently analyzed for their pertinence to LA. Of these, 113 publications were included in our systematic review. Among the included publications, 75 were conducted in a human setting and 38 in an animal setting. One article included both a case report and an animal experiment. One human study was published as two publications. The flow diagram of study selection is presented in figure 1 .

\section{Human studies}

\section{Randomized controlled trials}

One phase-II randomized controlled trial (unpublished, available as conference abstract at the time of writing) evaluated the efficacy of ILE on the pharmacokinetic properties of LA in 16 healthy volunteers ( 8 female and 8 male) aged $18-40$ years (Table 1$).(10,11)$ This was a doubleblind crossover study consisting of a first phase of habituation to LA with an infusion of lidocaine, followed by a second phase of either a continuous infusion of ropivacaine or levobupivacaine at $8 \mathrm{mg} / \mathrm{min}$ treated with either a bolus of $120 \mathrm{~mL}$ of $20 \%$ ILE or of $0.9 \%$ saline, administered two minutes after the start of the LA infusion. The primary outcome of interest was the duration of drug infusion (expressed as total dose) required to induce early clinical signs of neurotoxicity such as paresthesiae and a sensation of inebriation, as evaluated by an examiner blinded to the treatment. Secondary outcome measures were detection of sub-clinical seizure 


\section{AACT manuscript LA to edit for CTX}

activity based on electroencephalogram (EEG), duration of PR, QRS intervals based on electrocardiogram (EKG) and pharmacokinetics of local anesthetics (maximum concentration (Cmax) and area under the plasma concentration versus time curve (AUC)).

No significant difference in the total LA dose given to reach early signs of clinical toxicity was observed between ILE and control groups: Ropivacaine/ILE (75.7 mg $\pm 29.1 \mathrm{mg})$ or saline (81.7 $\mathrm{mg} \pm 22.3 \mathrm{mg})$ and Levobupivacaine/ILE $(69.4 \mathrm{mg} \pm 26.2 \mathrm{mg})$ or saline $(80.8 \mathrm{mg} \pm 31.7 \mathrm{mg})(\mathrm{p}$ $=0,61)$. The LA dose was provided at $8 \mathrm{mg} / \mathrm{min}$, and maximum allowed dose was $120 \mathrm{mg}$. Four of the 16 volunteers received the maximum dose of LA allowed in the protocol. No EEG abnormalities were seen. QRS prolongation was present at the end of the LA infusion as compared to baseline $(\mathrm{P}<0.001)$, but no significant difference was observed between the ILE and control groups ( $\mathrm{p}=0.68)$.(11) Small pharmacokinetic differences between groups, including a 25-30\% reduction in Cmax and a $20 \%$ increase in volume of distribution of the LA at a comparable mean dose, were not statistically significant and disappeared after 45 minutes.(10) The authors concluded that their study confirmed the lipid sink hypothesis in humans, but that no clinical efficacy of ILE could be observed in this systemic toxicity model, where a 3.8 msec prolongation in QRS was induced by the LA perfusion. No obvious risk of bias was identified from the research protocol

(https://clinicaltrials.gov/ct2/show/NCT01602250?term=toxalip\&rank=1), but concerns remain regarding indirectness (use of surrogate markers and uncertain generalizability to a poisoning context) and imprecision of the reported results due to the small sample size (potentially underpowered study). 


\section{AACT manuscript LA to edit for CTX}

No published peer-reviewed clinical controlled or observational studies were retrieved by our search.

\section{Human case reports}

There were 73 case reports and one case series, including 10 cases not individually reported elsewhere, that described the effect of ILE for treating LA toxicity.(1, 12-84) These articles involved 83 patients, aged from 2 days to 91 years, of whom two died and 81 (98\%) survived (Table 1). The local anesthetics involved, often in combination, are shown in Tables 1 and 2.

The most common lipid concentration administered in these publications was $20 \%$ (71 cases; $86 \%$ ), while a $10 \%$ concentration was used in one case,(35) and the lipid concentration used was not reported in the remaining 11 cases (Table 1).(17, 19, 23, 28, 40, 47, 51, 71) Lipid emulsion was administered as a bolus in $30(36 \%)$ cases, as a bolus followed by infusion in $34(41 \%)$ cases, and as an infusion without bolus in 8 cases (10\%). The dose regimen used was not specified or not reported in $11(13 \%)$ cases. The median bolus dose was $0.30 \mathrm{~g} / \mathrm{kg}$ (range 0.0015 $0.83 \mathrm{~g} / \mathrm{kg}$ ) and the median infusion dose was $1.9 \mathrm{~g} / \mathrm{kg} / \mathrm{hr}$ (range $0.015-6.0 \mathrm{~g} / \mathrm{kg} / \mathrm{hr}$ ). Overall, the total volume of lipid emulsion administered ranged from $9 \mathrm{~mL}$ to 2,480 $\mathrm{mL}$ (Table 1). The bolus dose in infants up to the age of one year was $1-2 \mathrm{mg} / \mathrm{kg}$ (Table 1 ). 


\section{AACT manuscript LA to edit for CTX}

In 52 cases $(63 \%)$ the lipid emulsion used was Intralipid ${ }^{\mathrm{TM}}$; Other formulations used were Medialipid $^{\mathrm{TM}}(\mathrm{n}=3,4 \%)$, Lipofundin $^{\mathrm{TM}}(\mathrm{n}=3,4 \%), \operatorname{Liposyn}^{\mathrm{TM}}(\mathrm{n}=2,2 \%)$, Lipovenoes $^{\mathrm{TM}}(\mathrm{n}=1$, $1 \%)$, Kabiven $^{\mathrm{TM}}(\mathrm{n}=1,1 \%)$, and Intralipos ${ }^{\mathrm{TM}}(\mathrm{n}=1,1 \%)$. In $20(24 \%)$ cases the lipid emulsion formulation was not reported (Table 1).

Sixty-nine (83\%) patients experienced toxicity following the use of local anesthetics for nerve blocks (Table 1), including 10 cases described in the single case series (Table 1).(19) Toxicity was also reported after intravenous $(\mathrm{n}=4 ; 5 \%),(25,32,51,74)$ subcutaneous $(\mathrm{n}=6 ; 7 \%),(12,19$, $29,33,44,54)$ intraosseous $(n=1 ; 1 \%),(31)$ topical $(n=1 ; 1 \%),(45)$ intraperitoneal $(n=2 ; 2 \%),(44$, $65)$ intraarticular $(n=1 ; 1 \%),(29)$ and intrapleural administration $(n=1 ; 1 \%)(39)$. Two routes of administration were involved in three cases $(4 \%)(29,44,65)$ and the route was not reported in one case $(1 \%)(40)$ (Table 1).

The most frequent toxic effects from LA reported were central nervous system (CNS) features including (but not limited to) CNS depression/coma or agitation $(n=45 ; 54 \%)$ and seizures $(n=49$; 59\%). Cardiovascular features included hypotension, hypertension, EKG changes and arrhythmias $(\mathrm{n}=39 ; 47 \%)$ and cardiac arrest $(\mathrm{n}=18 ; 22 \%)$; other non-cardiovascular symptoms $(\mathrm{n}=22 ; 27 \%)$ were also common (Table 1$)$.

In 14 cases $(17 \%),(21,35,37,42,44,46,48,51,52,61,63,65,74,80)$ ILE was the only treatment used for reversal of toxic effects and in $10(12 \%)$ of these resolution of symptoms was 


\section{AACT manuscript LA to edit for CTX}

reported.(21, 35, 42, 44, 46, 48, 52, 61, 65, 74) In $4(5 \%)$ cases the authors reported that it was unclear if effects were related to ILE. $(37,51,63,80)$ One case report did not comment on ILE effects (46) (Table 1).

Sixty case reports $(72 \%)$ described the use of ILE in combination with additional treatments. In $35(42 \%)$ cases lipid emulsion was used after failure of other treatments, in $6(7 \%)$ cases before other treatment, and in 16 (19\%) cases lipid emulsion was used concomitantly. The sequence of treatment was not reported in $3(4 \%)$ cases (Table 1).

Other treatments used included benzodiazepines or other sedatives $(n=41 ; 49 \%)$, vasopressors $(n=29 ; 35 \%)$, sodium bicarbonate $(n=7 ; 8 \%)$, antiarrhythmic drugs $(n=9 ; 11 \%)$, intravenous fluids $(n=5 ; 6 \%)$, and/or other treatments $(n=20 ; 24 \%)$. Three studies $(4 \%)$ reported other but unspecified treatments. Cardiopulmonary resuscitation (CPR) and/or intubation and/or ventilation were initiated in $35(42 \%)$ cases. In $3(4 \%)$ cases the patient was already intubated when features of LA toxicity appeared. Oxygen supply by mask was initiated in 9 cases (11\%). Cardiac defibrillation was reported in 4 cases (5\%). CPR and/or intubation were not required in $19(23 \%)$ cases and use of these procedures was not reported in 16 studies (19\%) (Table 1). Nine case reports did not state if any other treatments were performed (Table 1).

The authors of these case reports observed that ILE had a possible beneficial effect or was the cause of resolution of toxic features in 59 of cases (71\%). Four case reports (5\%) suggested no 


\section{AACT manuscript LA to edit for CTX}

benefit from ILE. In 10 case reports $(12 \%)$ it was unclear whether benefits were related to ILE or not; the effect of ILE was not described in 10 other cases (12\%) (Table 1).

\section{Animal studies}

Among the 38 publications using animal models, 29 (76\%) were randomized controlled studies (11 studies on rats, 14 on pigs, 2 on dogs, and 2 on rabbits)(17, 85-112), 3 (8\%) were observational studies ( 1 study on pigs, 1 on rats, 1 on rabbits)(113-115), 5 (13\%) were case series (1 study on rabbits and 4 on rats)(116-120) and one (3\%) was a case report (cat)(121). The animal studies are summarized in Table 3.

The local anesthetics studied the 38 studies included bupivacaine ( $\mathrm{n}=29$ studies, $76 \%$ ) levobupivacaine $(n=5,13 \%)$, lidocaine $(n=1,3 \%)$, mepivacaine $(n=1,3 \%)$ and ropivacaine $(n=4$, 11\%). Two studies combined two local anesthetics, bupivacaine/mepivacaine and levobupivacaine/ropivacaine (Table 3).

Lipid concentrations used were $20 \%(n=22,58 \%), 30 \%(n=8,21 \%)$ or not described $(n=2,5 \%)$. A bolus dose was used in $29(76 \%)$ studies and was followed by an infusion in $22(58 \%)$ studies. In three (8\%) studies an infusion was used without an initial bolus. The median bolus dose was $0.80 \mathrm{~g} / \mathrm{kg}$ or $4 \mathrm{~mL} / \mathrm{kg}$ of $20 \%$ ILE (range $0.20-3.0 \mathrm{~g} / \mathrm{kg}$ ) and median infusion dose was $6.0 \mathrm{~g} / \mathrm{kg} / \mathrm{hr}$ (range $0.60-54 \mathrm{~g} / \mathrm{kg} / \mathrm{hr}$ ). In 22 (58\%) studies, the lipid emulsion used was Intralipid ${ }^{\mathrm{TM}}$ while two studies used the medium chain/long chain triglyceride preparations Lipovenos ${ }^{\mathrm{TM}} \mathrm{MCT}(\mathrm{n}=2 / 5 \%)$ 


\section{AACT manuscript LA to edit for CTX}

or Medialipid ${ }^{\mathrm{TM}}(\mathrm{n}=1 / 3 \%)$. Other products used in one study each $(3 \%)$ were Lipovenos $^{\mathrm{TM}}$, Ivelip $^{\mathrm{TM}}$, SMOFLipid ${ }^{\mathrm{TM}}$, ClinOleic $^{\mathrm{TM}}$, and Liposyn II $^{\mathrm{TM}}$; an un-named Soybean oil emulsion was used in one study and the ILE formulation used was not reported in five studies (Table 3).

\section{Animal randomized controlled studies}

In 12 of the 29 randomized controlled studies, ILE was compared to the vasopressors epinephrine and/or vasopressin, either alone or in combination, or with vasopressors combined with ILE (Table 3).(88, 89, 91, 92, 97, 102-105, 107, 110, 111)

The ILE vs. epinephrine studies $(88,89,91,102-104,107,110,111)$, showed therapeutic benefit on survival or return to spontaneous circulation for ILE compared to epinephrine in four studies (14\% of the animal RCS) $(102-104,107)$ and for epinephrine+vasopressin in one study $(3 \%$ of the animal RCS).(105) There were comparable effects of ILE and epinephrine in five studies (17\% of the animal RCS).(88, $89,91,110,111)$

Combining epinephrine+ILE gave a better survival outcome compared to ILE alone in six studies ( $21 \%$ of the animal RCS $),(88,89,97,102,104,111)$ and of these, two studies concluded comparable effect of epinephrine and epinephrine+ILE.(102, 104)

In two studies (7\% of the animal RCS), the combination vasopressin+ILE did not improve survival over ILE alone and was not as effective as epinephrine or epinephrine+ILE 


\section{AACT manuscript LA to edit for CTX}

treatment.(102, 104) ILE was superior in one study (4\% of the animal RCS) comparing ILE with vasopressin alone or epinephrine+vasopressin.(92)

Lipid infusion was compared to crystalloids, either saline $(17,85-87,90,91,94,96,97,106-111)$ or Ringer's acetate(101) in 15 of the 29 randomized controlled studies. (Table 3) Nine (31\% of the animal RCS) studies showed therapeutic benefit $(85,87,90,91,94,97,106,109,110)$ and six $(21 \%)$ no benefit if ILE.(17, 86, 96, 101, 107, 111) In one study comparing ILE and saline, the control groups were different (electrically initiated ventricular fibrillation vs. LA induced venticular fibrillation); this study was therefore considered not useful for evaluating the effect of ILE.(108)

Various LA doses were evaluated in the included studies, and these doses were provided in varying units. These doses depended on the type of LA and the secondary outcome symptom severity. For bupivacaine, used in 22 (76\%) out of the 29 studies, $(86,87,90-97,100-106,108$ 112) the dose ranged from $1 \mathrm{mg} / \mathrm{kg} / \mathrm{min}$ to $10 \mathrm{mg} / \mathrm{kg}$ given over 10 seconds or $4-30 \mathrm{mg} / \mathrm{kg}$ (Table 3). Levobupivacaine was used in four studies $(14 \%),(88,89,98,99)$ with a dose of $500 \mathrm{mg} / \mathrm{hr}$ (88), $10 \mathrm{mg} / \mathrm{kg}(99)$ or $3-8.3 \mathrm{mg} / \mathrm{kg} / \mathrm{min}(89,98)$. Mepivacaine was used in one study $(3 \%)$ at an infusion rate of $6 \mathrm{mg} / \mathrm{kg} / \mathrm{min}$.(101) Ropivacaine was used in three studies $(10 \%),(17,85,107)$ at $1.5 \mathrm{mg} / \mathrm{kg} / \mathrm{min}$ as lowest infusion dose up to a maximum of $14.9 \pm 2.8 \mathrm{mg} / \mathrm{kg}$ given as a bolus (Table 3). 


\section{AACT manuscript LA to edit for CTX}

Resuscitation treatments were generally initiated immediately or within three minutes of the termination of LA administration (Table 3). Intravenous lipid emulsion was initiated immediately or within one minute in 19 studies $(17,85,87,90,92,94,96,98,100-104,106,110,116-119)$ or

less commonly up to three minutes (2 studies), $(97,105)$ four minutes ( 1 study),(86) 10 minutes (2 studies), $(109,111)$ or 20 minutes (1 study)(108) after termination of LA administration. The timings of administration of study treatments were not reported in three studies $(10 \%) .(88,91$, 107) Three studies (10\%) were not designed to evaluate the therapeutic effects of ILE alone, and either compared the use of ILE for toxicity caused by two different local anesthetics,(101) compared two types of ILE (long-chain triglyceride vs. long-chain and medium-chain triglyceride (100) or evaluated the myocardial tissue $\mathrm{pH} .(108)$ These studies were not considered generalizable to human poisoning and were therefore excluded from further analysis.

\section{Animal observational studies}

The three observational studies included in this review measured the LA dose required to cause death in $50 \%$ of the animals dosed (LD50),(115) compared the effect of ILE or saline on QRS widening using ILE or saline,(113) or compared ILE to hypertonic saline in combination with ILE.(114)

Intravenous lipid emulsion was initiated immediately or within two minutes of the termination of LA administration (Table 3). Bupivacaine was used at $4 \mathrm{mg} / \mathrm{kg}$ (113), $10 \mathrm{mg} / \mathrm{kg}$ (114) or at several different doses.(115) A benefit of ILE use was supported by the demonstration that lipid 


\section{AACT manuscript LA to edit for CTX}

infusion increased the bupivacaine LD50 in rats by $48 \%$, from 12.5 to $18.5 \mathrm{mg} / \mathrm{kg},(115)$ and by reversing the lengthening of QRS interval induced by the injection of bupivacaine in pigs.(113) (Table 3)

\section{Animal case reports and case series}

Only one animal case report was retrieved from our literature search, describing a cat suffering from toxicity after administration of lidocaine $140 \mathrm{mg}(20 \mathrm{mg} / \mathrm{kg}) .(121)$ The ILE infusion regimen was derived from clinical human studies, but the dose was reduced due to concerns about fluid overload. The authors reported a pronounced clinical response within 15 minutes of ILE initiation, with the cat becoming more responsive to stimuli and being able to hold its head up without assistance.

Five studies were included in this review as case series as the studies did not include a control group without ILE or included ILE in both study groups.(116-120) Thus, one study compared ILE plus the addition of three different doses of epinephrine,(116) one study compared the effect of ILE for either levobupivacaine or ropivacaine toxicity,(120) and three studies evaluated if the protective action of lipid emulsion was mediated through the fatty acid oxidation pathway(117, 118) or involved the opioid receptor.(119) Bupivacaine $10 \mathrm{mg} / \mathrm{kg}$ was the LA used in 4 of these 5 studies,(116-119) and levobupivacaine and ropivacaine were used in the other study each at a dose of $2 \mathrm{mg} / \mathrm{kg} / \mathrm{hr}$.(120) Intravenous lipid emulsion was initiated immediately or up to one minute after LA administration. 


\section{AACT manuscript LA to edit for CTX}

\section{Assessment of the quality of evidence}

Table 4 presents the summary estimates with associated Grading of Recommendations Assessment, Development and Evaluation (GRADE) ratings for the 2 human controlled studies reporting the effect of ILE on LA cardiotoxicity and neurotoxicity. All other evidence retrieved in this systematic review was rated as of very low quality; the human studies were seriously limited by their study designs (all uncontrolled studies preventing comparison with a control group, such as case series and case reports) and by the high likelihood of publication bias (especially with case reports), while animal studies were seriously limited by indirectness (resuscitation model lacking generalizability to humans) and imprecision (systematically underpowered studies).(122128) 


\section{AACT manuscript LA to edit for CTX}

\section{Discussion}

In our systematic review on the effect of ILE for acute LA toxicity, we identified animal and clinical studies that yielded a very low quality of evidence. Most randomized studies were conducted in animal settings with limited observation of test subjects after treatment and where no autopsies were performed or drug concentration measured. The human publications presenting the effect of ILE in severe LA toxicity were mainly case reports. Data from these studies and reports showed inconsistent benefits of ILE for the treatment of acute LA intoxication. Many also employed several treatments and, although this reflects what often happens in clinical practice, it makes the assessment of the specific effects of ILE difficult if not impossible.

A possible beneficial effect was reported in $71 \%$ of the human case reports, although this estimate of benefit is questionable due to the high risk of publication bias usually associated with this specific study design and due to the indirectness of the results caused by the absence of comparison to a control group. Furthermore, the only human controlled study showed no effect of ILE on mild LA toxicity. Thus, most of the useful evidence supporting a beneficial effect of ILE relies on animal studies. In controlled animal experiments and animal observational studies the effects of ILE were mainly based on cardiovascular variables, which are the most frequently observed adverse events with bupivacaine, the LA most often studied. Neurological symptoms could not be fully evaluated as the animals used were anesthetized during the experimental procedures. Results suggested improved efficacy for the reversal of LA cardiovascular toxicity with ILE alone compared to other treatments received alone in $48 \%$ of the controlled animal 


\section{AACT manuscript LA to edit for CTX}

studies, but reduced efficacy was seen in $28 \%$ of the these studies. The dose of LA given to induce toxicity in animals may not be comparable to the toxic dose known in humans and the amount of ILE given bolus often exceeded current recommendations.

When ILE was compared to other active treatments (vasopressors), inconsistent results were observed. There were 13 controlled animal studies favoring ILE alone, 6 studies favoring vasopressors and 7 studies favoring the combination of vasopressors and ILE. These studies are too heterogenous to allow a pooled analysis. The limited results of the available observational studies also suggested a possible clinical benefit from ILE alone or in combination with other resuscitative treatments with the same limitations to the human poisoning context as stated previously.

From the 8 animal experiments using vasopressors and ILE, results appears to be conflicting and in particular with the use of epinephrine. On the one hand, ILE appears to be associated with better hemodynamic outcomes in one study.(110) On the other hand, epinephrine alone, or the association of epinephrine and ILE, was better than ILE alone for survival or hemodynamic outcomes in five other studies in pigs and rats.(102-104, 107, 111). It is worth noting that the amount of epinephrine and ILE given were quite heterogenous and the formulation of ILE was not reported in one publication. Finally, two studies demonstrated no differences between epinephrine and ILE for mortality. $(88,97)$ 


\section{AACT manuscript LA to edit for CTX}

Thus, data from the human case reports and the animal studies provides weak evidence that that ILE may be effective in some cases of LA toxicity. However, there is no convincing evidence that this treatment is more effective than the use of vasopressors. These results also do not offer evidence to support which treatment should be instituted as first line therapy when cardiovascular toxicity arises after LA anesthetic administration.

\section{Limitations}

We performed a very broad search of the literature using appropriate eligibility criteria by considering all types of study design, including preclinical studies, but we may not have uncovered all studies reported in abstract form. A further potential limitation of our review is the inclusion of animal studies, which may not be generalizable to human cases of LA poisoning. The consideration of animal studies to support clinical practice may be perceived as inappropriate by some. However, several editorials and reviews used animal data to support the concept of ILE as an antidote. Our decision to consider such methodology was driven by our intention to be as exhaustive as possible in a field where very little research is conducted, and when it is done, this is often in a non-optimal context.

The human cases described were also heterogeneous regarding the LA involved, the severity of symptoms, the ILE dose, and the use of other treatments before ILE and we could not explore the potential impact of these discrepancies on the efficacy of the intervention. As stated in the 


\section{AACT manuscript LA to edit for CTX}

Methodology paper (9) the primary outcome of interest was survival. The included case reports showed $98 \%$ survival. However the validity of this finding is questionable because of likely reporting and publication bias . In most case reports the reversal of toxicity was thought to be related to ILE, however, some uncertainty remains as other treatments were often provided at the same time and effects could not be specifically distinguished. Furthermore, it is likely that cases that described negative outcome after ILE administration are underreported.

Our inability to deliver some of the results as intended in the Methodology paper (9), was principally due to unreported data, insufficient data, or the nature of the data, and also included the units used to specify the dose. Our intention was to extract or calculate the total amount of ILE in $\mathrm{g} / \mathrm{kg}$, but in many articles the information was insufficient, and therefore different units appears in tables 1 and 3. As mentioned reperted in the results section, most of the included studies and reports used $20 \%$ ILE, but a limitation to this review is that in 11 human case reports and two animal studies, the actual concentration of the ILE used was not reported.

A further limitation of the data was that it was not easy to obtain information if reported sequelae in human case reports referred to adverse effects from local anesthetics or from ILE. The details of sequelae that we were able to extract from data are included in table 1. 


\section{AACT manuscript LA to edit for CTX}

\section{Conclusions}

The currently available published evidence concerning the effect of ILE in severe LA toxicity is

limited to very low quality studies such as small animal experiments and human and animal case reports or series. It is possible that ILE may be effective in some cases of LA toxicity. However, there is currently no consistent evidence that ILE is more effective than vasopressors. The available evidence is insufficient to judge the combined effects of ILE and vasopressors and to determine whether one drug should precede the other in treating severe LA toxicity.

\section{Acknowledgements}

Ahmed Al-Sakha, Saad Al-Juma, Daniel Morris, Tudor Botnaru, Aftab Azad, Anne-Ericka Vermette-Marcotte, Nicholas Nacca and the other members of the lipid emulsion workgroup for full text article retrieval. Sarah Shiffert and Ellen Pak from AACT for arranging meetings and conference calls.

\section{Funding}

Dr Lavergne and Dr Turgeon are recipients of salary support awards from the Fonds de la Recherche du Québec - Santé (FRQS).

\section{Conflict of interest}

All members completed a conflict of interest form for AACT and received no honoraria. 


\section{AACT manuscript LA to edit for CTX}

Webcast conference and rooms for meeting were provided by AACT.

No member with a financial or academic conflict of interest preventing neutral assessment of the literature participated in the review (i.e. no committee member's livelihood or academic career is depending on a grant studying lipid emulsion in poisoning). 
AACT manuscript LA to edit for CTX

\section{Appendix 1: Medline (ovid) search strategy for lipid emulsion therapy effect}

1. exp Fat Emulsions, Intravenous/

2. lipid rescue.ti,ab,kw.

3. (lipid adj3 emulsi * ).mp.

4. (fat adj3 emulsi *).mp.

5. ((lipid or fat *) adj5 bolus).mp.

6. (lipid adj3 (resuscitat* or therap * or infus *)).mp.

7. (ILE adj5 (lipid * or emulsi * or fat*)).mp.

8. (IFE adj5 (lipid * or emulsi * or fat*)).mp.

9. (lipid adj3 $\operatorname{sink} *$ ).mp.

10. (lipid adj3 sequest *).mp.

11. intravenous * lipid * .ti,ab,kw.

12. intralipid *.mp.

13. or/1-12

14. exp Cardiovascular Agents/

15. exp Sodium Channel Blockers/

16. exp Calcium Channel Blockers/

17. exp Adrenergic beta-Antagonists/

18. ((sodium or $\mathrm{Na} *$ ) adj3 channel block *).ti,ab,kw.

19. ((calcium or $\left.\mathrm{Ca}^{*}\right)$ adj 3 channel block*).ti,ab,kw.

20. (beta adj3 block*).ti,ab,kw.

21. B-blocker.ti,ab,kw.

22. exp Central Nervous System Depressants/

23. exp Psychotropic Drugs/

24. exp Anti-Arrhythmia Agents/

25. local an?esthetic*.mp.

26. exp Amitriptyline/

27. amitriptyline.mp.

28. exp Bupropion/

29. bupropion.mp.

30. exp Chloroquine/

31. chloroquine.mp.

32. chlorpromazine.mp.

33. clomipramine.mp.

34. cocaine.mp.

35. exp Dothiepin/

36. (dosulepin or dothiepin).mp.

37. glyphosate.mp.

38. haloperidol.mp.

39. lamotrigine.mp.

40. olanzapine.mp. 


\section{AACT manuscript LA to edit for CTX}

41. propofol.mp.

42. quetiapine.mp.

43. exp Sertraline/

44. sertraline.ti,ab,kw.

45. zopiclone.mp.

46. ropivacaine.mp.

47. levobupivacaine.mp.

48. lignocaine.mp.

49. diazepam.mp.

50. exp Carnitine/

51. carnitine.ti,ab,kw.

52. exp Poisoning/

53. poison * .ti,ab,kw.

54. exp Noxae/ae, po [Adverse Effects, Poisoning]

55. po.fs.

56. ae.fs.

57. to.fs.

58. exp Street Drugs/

59. (lipophilic adj3 (drug * or toxin *)).ti,ab,kw.

60. overdos * .ti,ab,kw.

61. exp Antidotes/

62. antidote * .ti,ab,kw.

63. (toxic * or intoxic * or pharmacotoxic *).ti,ab,kw.

64. Resuscitation/

65. resuscitat* .ti,ab,kw.

66. or/14-65

67. 13 and 66 
AACT manuscript LA to edit for CTX

Figure 1: Selection of articles flow diagram

Search date December $15^{\text {th }} 2014$.

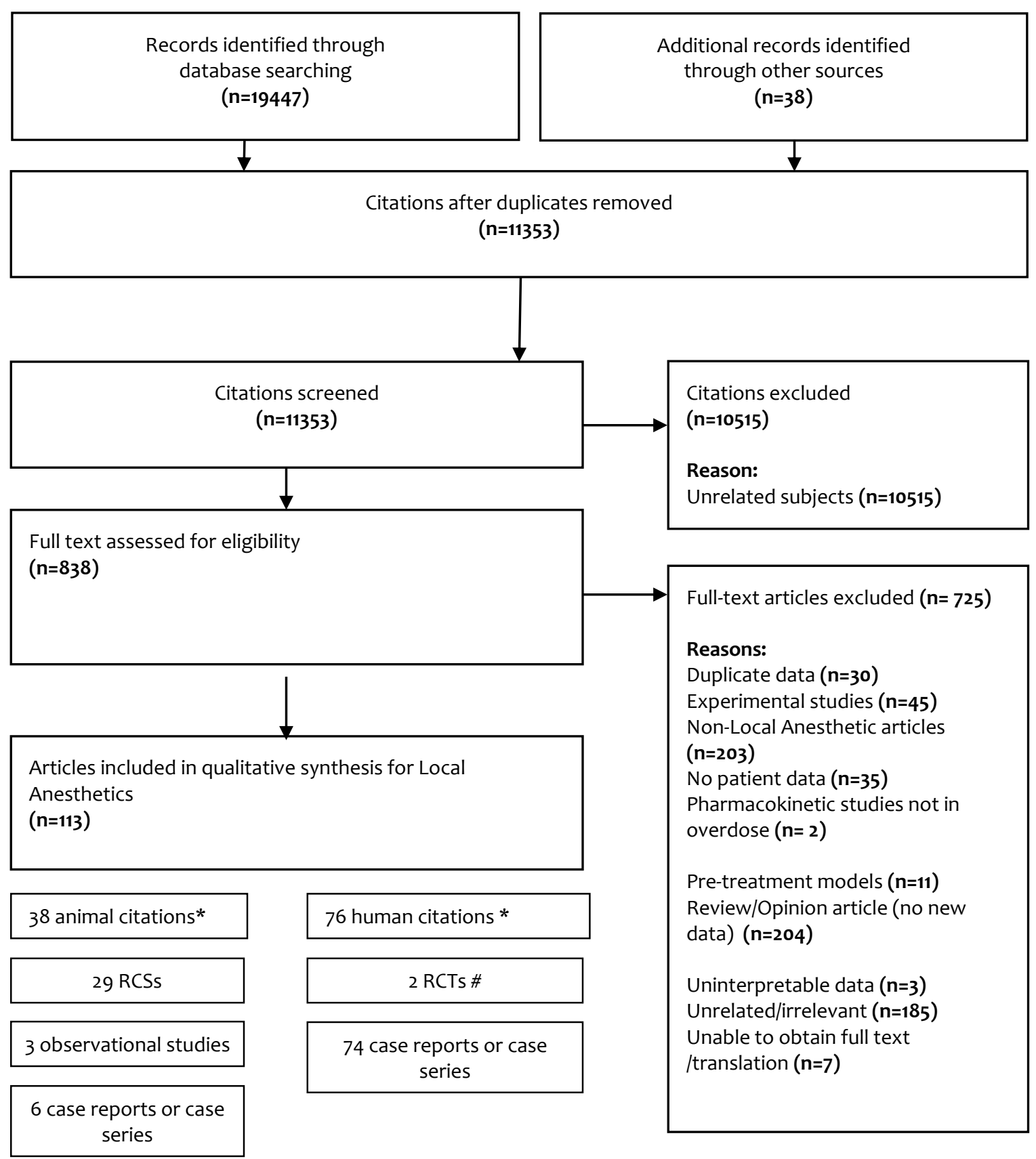




\section{AACT manuscript LA to edit for CTX}

*one citation included both one animal study and one human case report. \# The two citations covers one single study. 
AACT manuscript LA to edit for CTX

Table 1. Summary of the 16 volunteers from a crossover randomized controlled trial, and 83 patients from 73 case

\section{reports and one case series included in the systematic review.}

ACLS: Advanced cardiac life support, AV: Atrio-ventricular, BP: Blood Pressure, CNS: Central nervous system, CPR: Cardiopulmonary resuscitation, ICU: Intensive care unit, GCS: Glasgow coma score, HR: Heart rate, ILE: Intravenous lipid emulsion, LA: Local anesthetic, LCT: Long-chain triglyceride, MAP: Mean arterial pressure, MCT: Medium-chain triglyceride, NR: Not reported, PVC: Premature ventricular contractions, RCT: Randomized controlled trial, ROSC: Return of spontaneous circulation, VT: Ventricular tachycardia.

Note: Lidocaine and lignocaine are synonyms for the same compound, and the name lidocaine is used in the table.

\#: The total dose in $\mathrm{g} / \mathrm{kg}$ was infrequently available, and could only be calculated if bodyweight was reported.

: Available as abstracts only at the time of writing.

\begin{tabular}{|c|c|c|c|c|c|c|c|c|c|c|c|c|}
\hline Reference & $\begin{array}{l}\text { Study } \\
\text { type }\end{array}$ & $\begin{array}{l}\text { Age/sex, } \\
\text { weight }\end{array}$ & $\begin{array}{l}\text { Local } \\
\text { anesthetic and } \\
\text { dose }\end{array}$ & $\begin{array}{l}\log D \\
(129)\end{array}$ & $\begin{array}{l}\text { Route of } \\
\text { administration }\end{array}$ & Symptoms & ILE used & ILE dose\# & $\begin{array}{l}\text { ILE } \\
\text { only } \\
\text { used }\end{array}$ & $\begin{array}{l}\text { Other treatments } \\
\text { received, dose } \\
\text { included if } \\
\text { reported }\end{array}$ & ILE effect & Outcome \\
\hline \multicolumn{13}{|l|}{ RCT } \\
\hline \begin{tabular}{|l|} 
Dureau \\
2014/R010+R011 (10, \\
11) $\not$
\end{tabular} & $\begin{array}{l}\text { RCT, } \\
\text { cross- } \\
\text { over }\end{array}$ & $\begin{array}{l}\text { Age } 18-40 \\
\text { y/8F+8M }\end{array}$ & $\begin{array}{l}\text { Ropivacaine or } \\
\text { Levobupivacaine; } \\
\text { Continued infusion } \\
\text { doses } 8 \mathrm{mg} / \mathrm{min}, \\
\text { maximum } 120 \mathrm{mg} \text { or } \\
\text { until early signs of } \\
\text { toxicity such as } \\
\text { paresthesias or } \\
\text { inebriation sensation } \\
\text { reached. }\end{array}$ & $\begin{array}{l}4.21 \\
2.68\end{array}$ & Infusion & \begin{tabular}{|l} 
Neurologic impregnation \\
(parasthesia, inebriation) \\
QRS broadening at LA cessation
\end{tabular} & $\begin{array}{l}20 \% \\
\text { Intralipid }\end{array}$ & $120 \mathrm{~mL}$ bolus & Yes & $\begin{array}{l}\text { Saline }(120 \mathrm{ml}) \text { on two study } \\
\text { days (Control group) }\end{array}$ & $\begin{array}{l}\text { Study confirms the } \\
\text { Lipid Sink hypothesis in } \\
\text { humans, but unable to } \\
\text { demonstrate any } \\
\text { clinical benefit of ILE }\end{array}$ & $\begin{array}{l}4 \text { out of } 16 \text { volunteers } \\
\text { reached maximum dose. } \\
\text { Mean dose to reach mild } \\
\text { toxicity threshold was not } \\
\text { different in ILE vs control } \\
\text { groups ., }(75.7=/-29.1 \mathrm{mg} \\
\text { vs } 81.7=/-22.3 \mathrm{mg} \text { for } \\
\text { ropivacaine and } 69.4=/- \\
26.2 \mathrm{mg} \text { v } 80.8=/-31.7 \mathrm{mg} \\
\text { for levobupivacaine }\end{array}$ \\
\hline \multicolumn{13}{|l|}{ Case reports/series } \\
\hline AdMani, 2010 (12) & \begin{tabular}{|l|} 
Case \\
report
\end{tabular} & $\begin{array}{l}3 \mathrm{mth} / \mathrm{M}, 5.9 \\
\mathrm{~kg}\end{array}$ & $\begin{array}{l}\text { Bupivacaine } 25 \mathrm{mg}, \\
\text { Lidocaine } 100 \mathrm{mg}\end{array}$ & $\begin{array}{l}2.68 \\
1.26\end{array}$ & Subcutaneous & $\begin{array}{l}\text { Seizure. bradycardia with block } \\
\text { then Ventricular fibrillation and } \\
\text { Ventricular tachycardia }\end{array}$ & $20 \%$ & $\begin{array}{l}9 \mathrm{~mL}(0.31 \mathrm{~g} / \mathrm{kg}) \\
\text { bolus then } 0.25 \\
\mathrm{~mL} / \mathrm{kg} / \mathrm{min}(0.51 \\
\mathrm{g} / \mathrm{kg} / \mathrm{hr})\end{array}$ & No & \begin{tabular}{|l|} 
Dexamethasone $2 \mathrm{mg}$, \\
hydrocortisone $20 \mathrm{mg}$, \\
thiopental $5 \mathrm{mg}$ \\
Mechanical ventilation \\
\end{tabular} & \begin{tabular}{|l} 
Probably no effect \\
required benzodiapine \\
after seizures restarted \\
in ICU
\end{tabular} & $\begin{array}{l}\text { Survival, } \\
\text { no sequelae }\end{array}$ \\
\hline Al-Alami, 2011 (13) & \begin{tabular}{|l|} 
Case \\
report
\end{tabular} & $\begin{array}{l}16 \mathrm{y} / \mathrm{M}, \\
58 \mathrm{~kg}\end{array}$ & Ropivacaine $300 \mathrm{mg}$ & 4.21 & Nerve block & $\begin{array}{l}\text { Confusion, visual hallucinations } \\
\text { slurred speech tremor } \\
\text { Sinus tachycardia and } \\
\text { hypertension }\end{array}$ & $\begin{array}{l}20 \% \\
\text { Intralipid }\end{array}$ & $\begin{array}{l}0.0015 \mathrm{~g} / \mathrm{kg} \text { bolus } \\
\text { then } 0.015 \mathrm{~g} / \mathrm{kg} / \mathrm{hr} \\
\text { in } 3 \mathrm{hrs}\end{array}$ & No & Midazolam $0.5 \mathrm{mg}$ & $\begin{array}{l}\text { ILE was safe and } \\
\text { successful in reversing } \\
\text { LA-induced early CNS } \\
\text { and cardiac } \\
\text { abnormalities }\end{array}$ & $\begin{array}{l}\text { Survival, } \\
\text { no sequelae }\end{array}$ \\
\hline Aveline, 2010 (14) & \begin{tabular}{|l|} 
Case \\
report
\end{tabular} & $\begin{array}{l}52 \mathrm{y} / \mathrm{F}, \\
57 \mathrm{~kg}\end{array}$ & \begin{tabular}{|l} 
Lidocaine $400 \mathrm{mg}$, \\
Ropivacaine $112.5 \mathrm{mg}$
\end{tabular} & $\begin{array}{l}1.26 \\
4.21\end{array}$ & Nerve block & $\begin{array}{l}\text { GCS 7, agitated, confused, } \\
\text { jerking arms/head }\end{array}$ & \begin{tabular}{|l|}
$20 \%$ \\
Intralipid
\end{tabular} & $\begin{array}{l}100 \mathrm{~mL}(0.35 \mathrm{~g} / \mathrm{kg}) \\
\text { x2 bolus }\end{array}$ & No & \begin{tabular}{|l|} 
Midazolam 3mg, thiopental \\
300mg and suxamethonium \\
$80 \mathrm{mg}$ \\
Intubation
\end{tabular} & ILE not effective & $\begin{array}{l}\text { Survival, } \\
\text { no sequelae }\end{array}$ \\
\hline
\end{tabular}


AACT manuscript LA to edit for CTX

\begin{tabular}{|c|c|c|c|c|c|c|c|c|c|c|c|c|}
\hline Reference & $\begin{array}{l}\text { Study } \\
\text { type }\end{array}$ & $\begin{array}{l}\text { Age/sex, } \\
\text { weight }\end{array}$ & $\begin{array}{l}\text { Local } \\
\text { anesthetic and } \\
\text { dose }\end{array}$ & $\begin{array}{l}\log D \\
(129)\end{array}$ & $\begin{array}{l}\text { Route of } \\
\text { administration }\end{array}$ & Symptoms & ILE used & ILE dose\# & $\begin{array}{l}\text { ILE } \\
\text { only } \\
\text { used }\end{array}$ & $\begin{array}{l}\text { Other treatments } \\
\text { received, dose } \\
\text { included if } \\
\text { reported }\end{array}$ & ILE effect & Outcome \\
\hline Bazerbachi, 2013 (15) & \begin{tabular}{|l|} 
Case \\
report
\end{tabular} & $\begin{array}{l}57 \mathrm{y} / \mathrm{M}, \\
\text { weight NR }\end{array}$ & Ropivacaine $1540 \mathrm{mg}$ & 4.21 & Nerve block & $\begin{array}{l}\text { Lethargic, hallucinations } \\
\text { Bradycardia, wide QRS, } \\
\text { prolonged QT, ejection fraction } \\
20 \% \\
\text { Cardiac arrest } \\
\text { Tinnitus, dysgeusia } \\
\end{array}$ & $\begin{array}{l}20 \% \\
\text { Intralipid }\end{array}$ & Total dose $2480 \mathrm{~mL}$ & No & Plasmapheresis & $\begin{array}{l}\text { Suggest the failure of } \\
\text { ILE rescue. }\end{array}$ & Died from cardiac arrest \\
\hline Bilotta, 2012 (16) & \begin{tabular}{|l|} 
Case \\
report
\end{tabular} & $\begin{array}{l}53 \mathrm{y} / \mathrm{M}, \\
\text { weight NR }\end{array}$ & \begin{tabular}{|l|} 
Lidocaine $500 \mathrm{mg}$, \\
Ropivacaine $3000 \mathrm{mg}$
\end{tabular} & $\begin{array}{l}1.26 \\
4.21\end{array}$ & Nerve block & $\begin{array}{l}\text { AV block, HR 28/min, MAP } 40 \\
\text { mmHg }\end{array}$ & \begin{tabular}{|l|}
$20 \%$ \\
Intralipid
\end{tabular} & $\begin{array}{l}100 \mathrm{~mL} \text { bolus }(<5 \\
\mathrm{min} \text { ) then } 100 \mathrm{~mL} \text { in } \\
20 \mathrm{~min}\end{array}$ & No & $\begin{array}{l}\text { Atropine } 0.5 \mathrm{mg} \text {, } \\
\text { phenylephrine } 10 \mathrm{mg}\end{array}$ & Rapid beneficial effect & \begin{tabular}{|l|} 
Survival, \\
no sequelae
\end{tabular} \\
\hline $\begin{array}{l}\text { Buckenmaier, } 2012 \\
\text { (17) }\end{array}$ & \begin{tabular}{|l|} 
Case \\
report
\end{tabular} & $\begin{array}{l}29 \mathrm{y} / \mathrm{M}, \\
\text { weight NR }\end{array}$ & \begin{tabular}{|l} 
Ropivacaine, \\
Mepivacaine. \\
Bolus and continued \\
infusion doses of both, \\
total dose NR.
\end{tabular} & $\begin{array}{l}4.21 \\
1.40\end{array}$ & Nerve block & Unresponsive, cardiac arrest & Intralipid & $1 \mathrm{~mL} / \mathrm{kg} \times 3$ bolus & No & $\begin{array}{l}\text { Epinephrine, atropine, } \\
\text { amiodarone, calcium, } \\
\text { sodium bicarbonate, } \\
\text { magnesium, thrombolytic } \\
\text { therapy } \\
\text { CPR }\end{array}$ & NR & $\begin{array}{l}\text { Died from blast injuries } \\
\text { complicated by LA toxicity } \\
\text { resulting in a fatal cardiac } \\
\text { arrhythmia }\end{array}$ \\
\hline Calenda, 2009 (18) & \begin{tabular}{|l|} 
Case \\
report
\end{tabular} & $\begin{array}{l}72 \mathrm{y} / \mathrm{M}, \\
60 \mathrm{~kg}\end{array}$ & $\begin{array}{l}\text { Mepivacaine } 300 \mathrm{mg}, \\
\text { Ropivacaine } 112.5 \mathrm{mg}\end{array}$ & $\begin{array}{l}1.40 \\
4.21\end{array}$ & Nerve block & $\begin{array}{l}\text { Numb mouth/tongue } \\
\text { Seizures } \\
\text { Tachycardia (130/min) }\end{array}$ & $\begin{array}{l}20 \% \\
\text { Intralipid }\end{array}$ & \begin{tabular}{|l|}
$250 \mathrm{~mL}(0.83 \mathrm{~g} / \mathrm{kg})$ \\
bolus
\end{tabular} & No & $\begin{array}{l}\text { Midazolam } 5 \mathrm{mg} \text {, propofol } \\
150 \mathrm{mg} \text { (1st seizure); } \\
\text { Thiopental } 125 \mathrm{mg} \text { (2nd } \\
\text { seizure) } \\
\text { Mechanical ventilation } \\
\end{array}$ & $\begin{array}{l}\text { ILE not effective in } \\
\text { stopping 2nd seizure }\end{array}$ & $\begin{array}{l}\text { Survival, } \\
\text { no sequelae }\end{array}$ \\
\hline $\begin{array}{l}\text { Cave, } 2014 \text { (Lipid } \\
\text { Registry) (19) }\end{array}$ & \begin{tabular}{|l|} 
Case \\
series, \\
No 1
\end{tabular} & $\begin{array}{l}\text { Age NR/M, } \\
\text { weight NR }\end{array}$ & Lidocaine $560 \mathrm{mg}$ & 1.26 & Nerve block & \begin{tabular}{|l|} 
Hypertension \\
Drowsy
\end{tabular} & NR & NR & NR & NR & NR & $\begin{array}{l}\text { Survival, } \\
\text { sequelae NR }\end{array}$ \\
\hline $\begin{array}{l}\text { Cave, } 2014 \text { (Lipid } \\
\text { Registry) (19) }\end{array}$ & \begin{tabular}{|l} 
Case \\
series, \\
No 2
\end{tabular} & $\begin{array}{l}67 \mathrm{y} / \mathrm{F}, \\
49 \mathrm{~kg}\end{array}$ & $\begin{array}{l}\text { Lidocaine } 200 \mathrm{mg}, \\
\text { Bupivacaine } 75 \mathrm{mg}\end{array}$ & $\begin{array}{l}1.26 \\
2.68\end{array}$ & Nerve block & Seizure & \begin{tabular}{|l|}
$20 \%$ \\
Intralipid
\end{tabular} & \begin{tabular}{|l|}
$1.5 \mathrm{~mL} / \mathrm{kg}(0.30$ \\
$\mathrm{g} / \mathrm{kg}$ ) bolus then \\
$400 \mathrm{~mL}(4.9 \mathrm{~g} / \mathrm{kg} / \mathrm{hr})$ \\
in $20 \mathrm{~min}$. Total \\
dose $500 \mathrm{~mL}(2.04$ \\
$\mathrm{g} / \mathrm{kg})$
\end{tabular} & No & Midazolam & \begin{tabular}{|l|} 
ILE was thought to \\
have prevented death
\end{tabular} & $\begin{array}{l}\text { Survival, } \\
\text { sequelae NR }\end{array}$ \\
\hline \begin{tabular}{|l} 
Cave, 2014 (Lipid \\
Registry) (19)
\end{tabular} & \begin{tabular}{|l|} 
Case \\
series, \\
No 3
\end{tabular} & NR & $\begin{array}{l}\text { Mepivacaine } 900 \mathrm{mg}, \\
\text { Bupivacaine } 100 \mathrm{mg}\end{array}$ & $\begin{array}{l}1.40 \\
2.68\end{array}$ & Nerve block & $\begin{array}{l}\text { Decreased level of } \\
\text { consciousness }\end{array}$ & NR & NR & NR & NR & NR & \begin{tabular}{|l|} 
Survival, \\
sequelae NR
\end{tabular} \\
\hline $\begin{array}{l}\text { Cave, } 2014 \text { (Lipid } \\
\text { Registry)(19) }\end{array}$ & \begin{tabular}{|l|} 
Case \\
series, \\
No 4
\end{tabular} & $\begin{array}{l}68 \mathrm{y} / \mathrm{M}, \\
75 \mathrm{~kg}\end{array}$ & Ropivacaine $200 \mathrm{mg}$ & 4.21 & Nerve block & \begin{tabular}{|l|} 
Seizure \\
Cardiac arrest
\end{tabular} & \begin{tabular}{|l|}
$20 \%$ \\
Intralipid
\end{tabular} & \begin{tabular}{|l|}
$100 \mathrm{~mL}(0.27 \mathrm{~g} / \mathrm{kg})$ \\
$\times 3$ bolus. \\
Total dose $300 \mathrm{~mL}$ \\
$(0.80 \mathrm{~g} / \mathrm{kg})$ \\
\end{tabular} & No & $\begin{array}{l}\begin{array}{l}\text { Midazolam, epinephrine, } \\
\text { sodium bicarbonate, } \\
\text { magnesium, and } \\
\text { hydrocortisone }\end{array} \\
\end{array}$ & NR & \begin{tabular}{|l|} 
Survival, \\
sequelae NR
\end{tabular} \\
\hline \begin{tabular}{|l} 
Cave, 2014 (Lipid \\
Registry) (19)
\end{tabular} & \begin{tabular}{|l} 
Case \\
series, \\
No 5
\end{tabular} & $\begin{array}{l}69 \mathrm{y} / \mathrm{F}, \\
80 \mathrm{~kg}\end{array}$ & Bupivacaine $150 \mathrm{mg}$ & 2.68 & Nerve block & \begin{tabular}{|l|} 
Seizure \\
Cardiovascular collapse
\end{tabular} & \begin{tabular}{|l|}
$20 \%$ \\
Intralipid
\end{tabular} & $\begin{array}{l}1.5 \mathrm{~mL} / \mathrm{kg}(0.30 \\
\mathrm{g} / \mathrm{kg} \text { bolus then } \\
400 \mathrm{~mL} / \mathrm{hr}(1.0 \\
\mathrm{g} / \mathrm{kg} / \mathrm{hr}) \\
\text { Total dose } 500 \mathrm{~mL} \\
(1.25 \mathrm{~g} / \mathrm{kg})\end{array}$ & NR & NR & \begin{tabular}{|l|} 
ILE was thought to \\
have prevented death
\end{tabular} & \begin{tabular}{|l|} 
Survival, \\
sequelae NR
\end{tabular} \\
\hline
\end{tabular}


AACT manuscript LA to edit for CTX

\begin{tabular}{|c|c|c|c|c|c|c|c|c|c|c|c|c|}
\hline Reference & $\begin{array}{l}\text { Study } \\
\text { type }\end{array}$ & $\begin{array}{l}\text { Age/sex, } \\
\text { weight }\end{array}$ & $\begin{array}{l}\text { Local } \\
\text { anesthetic and } \\
\text { dose }\end{array}$ & $\left|\begin{array}{l}\log D \\
(129)\end{array}\right|$ & $\begin{array}{l}\text { Route of } \\
\text { administration }\end{array}$ & Symptoms & ILE used & ILE dose\# & $\begin{array}{l}\text { ILE } \\
\text { only } \\
\text { used }\end{array}$ & $\begin{array}{l}\text { Other treatments } \\
\text { received, dose } \\
\text { included if } \\
\text { reported }\end{array}$ & ILE effect & Outcome \\
\hline $\begin{array}{l}\text { Cave, } 2014 \text { (Lipid } \\
\text { Registry) (19) }\end{array}$ & \begin{tabular}{|l} 
Case \\
series, \\
No 6 \\
\end{tabular} & NR & Bupivacaine $50 \mathrm{mg}$ & 2.68 & Nerve block & Seizure & NR & NR & NR & NR & NR & \begin{tabular}{|l|}
$\begin{array}{l}\text { Survival, } \\
\text { sequelae NR }\end{array}$ \\
\end{tabular} \\
\hline $\begin{array}{l}\text { Cave, } 2014 \text { (Lipid } \\
\text { Registry) (19) }\end{array}$ & \begin{tabular}{|l|} 
Case \\
series, \\
No 7 \\
\end{tabular} & NR & Bupivacaine $100 \mathrm{mg}$ & 2.68 & Nerve block & Seizure & NR & NR & NR & NR & NR & $\begin{array}{l}\text { Survival, } \\
\text { sequelae NR }\end{array}$ \\
\hline \begin{tabular}{|l} 
Cave, 2014 (Lipid \\
Registry) (19)
\end{tabular} & \begin{tabular}{|l|} 
Case \\
series, \\
No 8
\end{tabular} & $\begin{array}{l}30 \mathrm{y} / \mathrm{M}, \\
81 \mathrm{~kg}\end{array}$ & Bupivacaine $100 \mathrm{mg}$ & 2.68 & Nerve block & Seizure & $\begin{array}{l}20 \%, \\
\text { Lipofundin }\end{array}$ & $\begin{array}{l}1.5 \mathrm{~mL} / \mathrm{kg}(0.30 \\
\mathrm{g} / \mathrm{kg} \text { ) bolus then } 15 \\
\mathrm{~mL} / \mathrm{min}(2.22 \\
\mathrm{g} / \mathrm{kg} / \mathrm{hr}) \\
\text { Total dose } 640 \mathrm{~mL} \\
1.58 \mathrm{~g} / \mathrm{kg})\end{array}$ & NR & NR & $\begin{array}{l}\text { ILE was thought to } \\
\text { have prevented death }\end{array}$ & $\begin{array}{l}\text { Survival, } \\
\text { sequelae NR }\end{array}$ \\
\hline $\begin{array}{l}\text { Cave, } 2014 \text { (Lipid } \\
\text { Registry) (19) }\end{array}$ & \begin{tabular}{|l|} 
Case \\
series, \\
No 9
\end{tabular} & $\begin{array}{l}47 \mathrm{y} / \mathrm{F}, \\
60 \mathrm{~kg}\end{array}$ & Bupivacaine $187.5 \mathrm{mg}$ & 2.68 & Subcutaneous & $\begin{array}{l}\text { Decreased level of } \\
\text { consciousness }\end{array}$ & \begin{tabular}{|l|}
$20 \%$ \\
Intralipid
\end{tabular} & $\begin{array}{l}1.5 \mathrm{~mL} / \mathrm{kg}(0.30 \\
\mathrm{g} / \mathrm{kg} \text { ) bolus then } 13 \\
\mathrm{~mL} / \mathrm{min}(2.58 \\
\mathrm{g} / \mathrm{kg} / \mathrm{hr}) \\
\mathrm{Total} \text { lose } 900 \mathrm{~mL} \\
(3.0 \mathrm{~g} / \mathrm{kg})\end{array}$ & NR & NR & NR & $\begin{array}{l}\text { Survival, } \\
\text { sequelae NR }\end{array}$ \\
\hline $\begin{array}{l}\text { Cave, } 2014 \text { (Lipid } \\
\text { Registry) (19) }\end{array}$ & \begin{tabular}{|l|} 
Case \\
series, \\
No 10
\end{tabular} & $\begin{array}{l}75 \mathrm{y} / \mathrm{F}, \\
57 \mathrm{~kg}\end{array}$ & Bupivacaine $1595 \mathrm{mg}$ & 2.68 & Nerve block & Seizure & \begin{tabular}{|l|}
$20 \%$, \\
Intralipid
\end{tabular} & $\begin{array}{l}1.5 \mathrm{~mL} / \mathrm{kg}(0.30 \\
\mathrm{g} / \mathrm{kg} \text { ) bolus then } \\
870 \mathrm{~mL} / \mathrm{hr}(3.05 \\
\mathrm{g} / \mathrm{kg} / \mathrm{hr}) \\
\text { Total dose } 587 \mathrm{~mL} \\
1.95 \mathrm{~g} / \mathrm{kg})\end{array}$ & NR & NR & NR & \begin{tabular}{|l|} 
Survival, \\
sequelae NR
\end{tabular} \\
\hline $\begin{array}{l}\text { Charbonneau, } 2009 \\
\text { (20) }\end{array}$ & \begin{tabular}{|l|} 
Case \\
report
\end{tabular} & $\begin{array}{l}19 \mathrm{y} / \mathrm{sex} \mathrm{NR}, \\
67 \mathrm{~kg}\end{array}$ & Mepivacaine 1000 mg & 1.40 & Nerve block & $\begin{array}{l}\text { Dysarthria, myoclonia, } \\
\text { confusion }\end{array}$ & $\begin{array}{l}20 \%, \\
\text { Medialipid }\end{array}$ & $\begin{array}{l}100 \mathrm{~mL}(0.30 \mathrm{~g} / \mathrm{kg}) \\
\text { bolus }\end{array}$ & No & $\begin{array}{l}\text { Midazolam } 1 \mathrm{mg}, \\
\text { clonazepam } 1 \mathrm{mg}\end{array}$ & \begin{tabular}{|l|}
$\begin{array}{l}\text { Efficacy was immediate } \\
\text { and complete }\end{array}$ \\
\end{tabular} & \begin{tabular}{|l|} 
Survival, \\
no sequelae
\end{tabular} \\
\hline \begin{tabular}{|l} 
Contargyris, 2012 \\
(21)
\end{tabular} & \begin{tabular}{|l|} 
Case \\
report
\end{tabular} & \begin{tabular}{|l|}
$26 \mathrm{y} / \mathrm{F}, 34 \mathrm{w}$ \\
pregnant, \\
$51 / 58 \mathrm{~kg}$ \\
\end{tabular} & $\begin{array}{l}\text { Bupivacaine } 7 \mathrm{mg}, \\
\text { Ropivacaine } 90 \mathrm{mg}\end{array}$ & $\begin{array}{l}2.68 \\
4.21\end{array}$ & Nerve block & $\begin{array}{l}\text { headache, metallic taste, } \\
\text { hallucinations }\end{array}$ & \begin{tabular}{|l|}
$20 \%$, \\
Intralipid
\end{tabular} & $\begin{array}{l}200 \mathrm{~mL}(0.78 \mathrm{~g} / \mathrm{kg} \\
\text { mother) bolus }\end{array}$ & Yes & NR & $\begin{array}{l}\text { Resolution of } \\
\text { symptoms }\end{array}$ & $\begin{array}{l}\text { Survival, } \\
\text { sequelae NR }\end{array}$ \\
\hline Cordell, 2010 (22) & \begin{tabular}{|l|} 
Case \\
report
\end{tabular} & $\begin{array}{l}17 \mathrm{y} / \mathrm{F}, \\
\text { weight NR }\end{array}$ & Bupivacaine 75 mg & 2.68 & Nerve block & \begin{tabular}{|l} 
Seizure \\
Tachycardia (180/min)
\end{tabular} & $\begin{array}{l}20 \%, \\
\text { brand NR }\end{array}$ & $\begin{array}{l}100 \mathrm{~mL} \times 3 \text { bolus, } \\
\text { then infusion. Total } \\
\text { dose NR }\end{array}$ & No & $\begin{array}{l}\text { Midazolam } 2 \mathrm{mg} \text {, propofol } \\
100 \mathrm{mg} \text { and epinephrine } 1 \\
\mathrm{mg} \\
\mathrm{CPR} \text {, intubation }\end{array}$ & $\begin{array}{l}\text { Resolution of cardiac } \\
\text { and neurologic } \\
\text { symptoms }\end{array}$ & $\begin{array}{l}\text { Survival, } \\
\text { no sequelae }\end{array}$ \\
\hline Dacosta, $2009(23) \not$ & \begin{tabular}{|l} 
Case \\
report
\end{tabular} & $\begin{array}{l}44 \mathrm{y} / \mathrm{F}, \\
104 \mathrm{~kg}\end{array}$ & \begin{tabular}{|l} 
Lidocaine $150 \mathrm{mg}$ \\
Bupivacaine $200 \mathrm{mg}$
\end{tabular} & $\begin{array}{l}1.26 \\
2.68\end{array}$ & Nerve block & $\begin{array}{l}\text { Metalic taste } \\
\text { Sinus bradycardia }(34 / \mathrm{min}), \\
\text { hypotension }(80 / 45 \mathrm{mmHg})\end{array}$ & NR & $100 \mathrm{ml}$ in $10 \mathrm{~min}$. & No & $\begin{array}{l}\text { Atropine } 2 \mathrm{mg} \text {, ephedrine } 5 \\
\mathrm{mg} \text {, saline } 500 \mathrm{ml}\end{array}$ & $\begin{array}{l}\text { Resolution of cardiac } \\
\text { symptoms in } 15 \mathrm{~min} .\end{array}$ & $\begin{array}{l}\text { Survival, } \\
\text { no sequelae }\end{array}$ \\
\hline Diaz, 2012 (24) & \begin{tabular}{|l|} 
Case \\
report
\end{tabular} & $\begin{array}{l}\text { Adult/F, } \\
75 \mathrm{~kg}\end{array}$ & \begin{tabular}{|l|} 
Levobupivicaine 34.25 \\
mg, Lidocaine $340 \mathrm{mg}$
\end{tabular} & $\begin{array}{l}2.68 \\
1.26\end{array}$ & Nerve block & $\begin{array}{l}\text { Somnolent, developed tremor, } \\
\text { nystagmus and became } \\
\text { comatose } \\
\text { Decrease in blood pressure } \\
\text { Nausea }\end{array}$ & $\begin{array}{l}20 \% \\
\text { Medialipid } \\
\text { (MCT/LCT) }\end{array}$ & $\begin{array}{l}100 \mathrm{~mL}(0.27 \mathrm{~g} / \mathrm{kg}) \\
\text { bolus then } 400 \mathrm{~mL} \\
(0.53 \mathrm{~g} / \mathrm{kg} / \mathrm{hr}) \text { in } 2 \\
\text { hrs }\end{array}$ & No & \begin{tabular}{|l} 
Phenylephrine, ondansetron \\
$4 \mathrm{mg}$, sufentanil $20.5 \mathrm{mcg}$, \\
clonidine $138 \mathrm{mcg}$
\end{tabular} & $\begin{array}{l}\text { Resolution of cardiac } \\
\text { and neurologic } \\
\text { symptoms }\end{array}$ & $\begin{array}{l}\text { Survival, } \\
\text { no sequelae }\end{array}$ \\
\hline
\end{tabular}


AACT manuscript LA to edit for CTX

\begin{tabular}{|c|c|c|c|c|c|c|c|c|c|c|c|c|}
\hline Reference & \begin{tabular}{|l} 
Study \\
type
\end{tabular} & $\begin{array}{l}\text { Age/sex, } \\
\text { weight }\end{array}$ & $\begin{array}{l}\text { Local } \\
\text { anesthetic and } \\
\text { dose }\end{array}$ & $\begin{array}{l}\log D \\
(129)\end{array}$ & $\begin{array}{l}\text { Route of } \\
\text { administration }\end{array}$ & Symptoms & ILE used & ILE dose\# & \begin{tabular}{|l|} 
ILE \\
only \\
used
\end{tabular} & $\begin{array}{l}\text { Other treatments } \\
\text { received, dose } \\
\text { included if } \\
\text { reported }\end{array}$ & ILE effect & Outcome \\
\hline Dix, 2011 (25) & \begin{tabular}{|l|} 
Case \\
report
\end{tabular} & $\begin{array}{l}57 \mathrm{y} / \mathrm{M}, \\
\text { weight NR }\end{array}$ & $\begin{array}{l}\text { Lidocaine } 120 \mathrm{mg}+2 \\
\mathrm{mg} / \mathrm{min} \text { infusion, total } \\
\text { dose NR }\end{array}$ & 1.26 & \begin{tabular}{|l} 
Intravenous \\
\end{tabular} & $\begin{array}{l}\text { Somnolent, confused, } \\
\text { unresponsive } \\
\text { QRS widening, suffered from } \\
\text { cardiovascular disease already- } \\
\text { Pulseless, electromechanical } \\
\text { dissociation } \\
\text { Tremor, difficulty performing } \\
\text { cerebellar testing }\end{array}$ & \begin{tabular}{|l|}
$20 \%$ \\
Intralipid
\end{tabular} & $\begin{array}{l}1 \mathrm{~mL} / \mathrm{kg} \text { bolus then } \\
0.25 \mathrm{~mL} / \mathrm{kg} / \mathrm{min} \text { in } \\
30 \mathrm{~min}\end{array}$ & No & $\begin{array}{l}\text { Epinephrine, amiodarone, } \\
\text { magnesium sulfate, calcium } \\
\text { gluconate, and sodium } \\
\text { bicarbonate, dopamine } 7 \\
\mathrm{mcg} / \mathrm{kg} / \mathrm{min} \\
\mathrm{CPR}\end{array}$ & $\begin{array}{l}\text { Resolution of cardiac } \\
\text { symptoms }\end{array}$ & \begin{tabular}{|l|} 
Survival, \\
no sequelae
\end{tabular} \\
\hline Egan 2013 (26) д & \begin{tabular}{|l|} 
Case \\
report
\end{tabular} & $\begin{array}{l}38 \mathrm{y} / \mathrm{F}, \\
62 \mathrm{~kg}\end{array}$ & $\begin{array}{l}\text { Ropivacaine } 100 \mathrm{mg}, \\
\text { Lidocaine } 200 \mathrm{mg}\end{array}$ & $\begin{array}{l}4.21 \\
2.26\end{array}$ & Nerve block & $\begin{array}{l}\text { Grand mal seizures } 1 \text { minutes } \\
\text { post injection } \\
\text { Coma }\end{array}$ & $\begin{array}{l}20 \% \\
\text { Intralipid }\end{array}$ & $\begin{array}{l}100 \mathrm{~mL}(0.32 \mathrm{~g} / \mathrm{kg}) \\
\text { bolus then } 0.25 \\
\mathrm{mg} / \mathrm{kg} / \mathrm{hr} \text { infusion, } \\
\text { duration NR }\end{array}$ & No & $\begin{array}{l}\text { Midazolam }>2 \mathrm{mg} \\
\text { Intubation }\end{array}$ & \begin{tabular}{|l|} 
Resolution of seizure \\
unclear timing \\
Resolution of coma 30 \\
minutes later
\end{tabular} & $\begin{array}{l}\text { Survival, } \\
\text { no sequelae }\end{array}$ \\
\hline Espinet, 2009 (27) & \begin{tabular}{|l|} 
Case \\
report
\end{tabular} & $\begin{array}{l}36 \mathrm{y} / \mathrm{M}, \\
80 \mathrm{~kg}\end{array}$ & $\begin{array}{l}\text { Bupivacaine } 100 \mathrm{mg}, \\
\text { Lidocaine } 100 \mathrm{mg}\end{array}$ & \begin{tabular}{|l|}
2.68 \\
1.26
\end{tabular} & Nerve block & \begin{tabular}{|l|} 
Perioral tingling, headache, \\
diziness, light headedness, \\
diplopia \\
Tachycardia (153/min), BP \\
180/110mmHg, ST depression
\end{tabular} & \begin{tabular}{|l|}
$20 \%$, \\
Intralipid
\end{tabular} & $\begin{array}{l}100 \mathrm{~mL}(0.25 \mathrm{~g} / \mathrm{kg}) \\
\times 2 \text { bolus then } 100 \\
\mathrm{~mL}(0.25 \mathrm{~g} / \mathrm{kg} / \mathrm{hr}) \text { in } \\
1 \text { hour }\end{array}$ & No & $\begin{array}{l}\text { Crystalloid (Hartmann's } \\
\text { solution) } 1 \mathrm{~L} \\
\text { Oxygen }\end{array}$ & $\begin{array}{l}\text { Resolution of cardiac } \\
\text { and neurologic } \\
\text { symptoms }\end{array}$ & \begin{tabular}{|l} 
Survival, \\
no sequelae
\end{tabular} \\
\hline Etesse, 2011 (28) & \begin{tabular}{|l|} 
Case \\
report
\end{tabular} & $\begin{array}{l}23 \mathrm{y} / \mathrm{F}, 38 \mathrm{w} \\
\text { pregnant, } \\
\text { weight NR }\end{array}$ & Ropivacaine $46 \mathrm{mg}$ & 4.21 & Nerve block & $\begin{array}{l}\text { Visual hallucination, nausea } \\
7 \text { hour prior to development of } \\
\text { status epilepticus } \\
\text { Hypertension }\end{array}$ & NR & $100 \mathrm{~mL}$ & No & $\begin{array}{l}\text { Midazolam } 2 \mathrm{mg}, \\
\text { magnesium sulfate } 1 \mathrm{~g} \text { in } 20 \\
\text { min and then } 1 \mathrm{~g} / \mathrm{hr} \\
\text { Oxygen }\end{array}$ & \begin{tabular}{|l|} 
Resolution of \\
neurologic symptoms
\end{tabular} & $\begin{array}{l}\text { Survival, } \\
\text { no sequelae }\end{array}$ \\
\hline \begin{tabular}{|l|} 
Fenten 2014 (29) \\
\end{tabular} & \begin{tabular}{|l|} 
Case \\
report
\end{tabular} & $\begin{array}{l}67 \mathrm{y} / \mathrm{F}, \\
\text { weight NR }\end{array}$ & Ropivacaine $400 \mathrm{mg}$ & 4.21 & $\begin{array}{l}\text { Intra articular/ } \\
\text { Subcutaneous }\end{array}$ & $\begin{array}{l}\text { Chest pain } \\
\text { Coma } \\
\text { Seizure }\end{array}$ & $\begin{array}{l}20 \% \text {, brand } \\
\text { NR }\end{array}$ & \begin{tabular}{|l|} 
Infusion \\
Unknown duration
\end{tabular} & No & $\begin{array}{l}\text { Nitroglycerin (spray), } \\
\text { metoprolol } 5 \mathrm{mg}, \\
\text { midazolam } 1 \mathrm{mg} \text { boluses } \\
\text { Oxygen }\end{array}$ & $\begin{array}{l}\text { Resolution of initial } \\
\text { seizure but recurrence } \\
\text { of seizures and } \\
\text { twitching for } 5.5 \mathrm{hr} \\
\text { after }\end{array}$ & $\begin{array}{l}\text { Survival, } \\
\text { no sequelae }\end{array}$ \\
\hline Foxall, 2007 (30) & \begin{tabular}{|l|} 
Case \\
report
\end{tabular} & $\begin{array}{l}75 \mathrm{y} / \mathrm{F}, \\
85 \mathrm{~kg}\end{array}$ & $\begin{array}{l}\text { Levobupivacaine } 100 \\
\mathrm{mg}\end{array}$ & 2.68 & Nerve block & $\begin{array}{l}\text { Unresponsiveness } \\
\text { Seizures } \\
\text { QRS widening; Suffered from } \\
\text { cardiovascular disease already } \\
\text { Groaned }\end{array}$ & $\begin{array}{l}20 \% \\
\text { Intralipid }\end{array}$ & $\begin{array}{l}100 \mathrm{~mL}(0.24 \mathrm{~g} / \mathrm{kg}) \\
\text { bolus in } 5 \mathrm{~min}\end{array}$ & No & $\begin{array}{l}\text { Metaraminol } 0.5 \mathrm{mg}, \\
\text { propofol } 80 \mathrm{mg}, \\
\text { suxamethonium } 100 \mathrm{mg} \\
\text { Oxygen, intubation }\end{array}$ & $\begin{array}{l}\text { Resolution of cardiac } \\
\text { symptoms }\end{array}$ & \begin{tabular}{|l|} 
Survival, \\
no sequelae
\end{tabular} \\
\hline French, 2012 (31) & \begin{tabular}{|l|} 
Case \\
report
\end{tabular} & $\begin{array}{l}11 \mathrm{mth} / \mathrm{M}, \\
9.9 \mathrm{~kg}\end{array}$ & Lidocaine $100 \mathrm{mg}$ & 1.26 & Intraosseous & Status epilepticus & $\begin{array}{l}20 \%, \\
\text { Intralipid }\end{array}$ & $\begin{array}{l}12 \mathrm{~mL}(0.24 \mathrm{~g} / \mathrm{kg}) \\
\text { bolus }\end{array}$ & No & Lorazepam $0.1 \mathrm{mg} / \mathrm{kg}$ & NR & \begin{tabular}{|l|} 
Survival, \\
no sequelae
\end{tabular} \\
\hline \begin{tabular}{|l|} 
Fuzaylov, 2010 (32) \\
\end{tabular} & \begin{tabular}{|l|} 
Case \\
report
\end{tabular} & $\begin{array}{l}13 \mathrm{y} / \mathrm{F}, \\
50 \mathrm{~kg}\end{array}$ & Bupivacaine $25 \mathrm{mg}$ & 2.68 & Intravenous & $\begin{array}{l}\text { Decreased BP (from } 90 \text { to } \\
60 \mathrm{mmHg} \text { ) and broad complex } \\
\text { ventricular tachycardia }\end{array}$ & \begin{tabular}{|l|}
$20 \%$, \\
Intralipid
\end{tabular} & $\begin{array}{l}100 \mathrm{~mL}(0.4 \mathrm{~g} / \mathrm{kg}) \\
\text { bolus }\end{array}$ & No & $\begin{array}{l}\text { Saline } 500 \mathrm{~mL} \text {. Epinephrine } \\
10 \mathrm{mcg} \times 2 \text { bolus }+0.1 \\
\mathrm{mcg} / \mathrm{kg} / \mathrm{min} \text { infusion, } \\
\text { dopamine } 10 \mathrm{mcg} / \mathrm{kg} / \mathrm{min} \\
\text { infusion } \\
\text { CPR }\end{array}$ & \begin{tabular}{|l|} 
Possible effect in \\
resolution of symptoms
\end{tabular} & $\begin{array}{l}\text { Survival, } \\
\text { pulmonary edema, resolved } \\
\text { day } 4\end{array}$ \\
\hline
\end{tabular}


AACT manuscript LA to edit for CTX

\begin{tabular}{|c|c|c|c|c|c|c|c|c|c|c|c|c|}
\hline Reference & $\begin{array}{l}\text { Study } \\
\text { type }\end{array}$ & $\begin{array}{l}\text { Age/sex, } \\
\text { weight }\end{array}$ & $\begin{array}{l}\text { Local } \\
\text { anesthetic and } \\
\text { dose }\end{array}$ & $\begin{array}{l}\log D \\
(129)\end{array}$ & $\begin{array}{l}\text { Route of } \\
\text { administration }\end{array}$ & Symptoms & ILE used & ILE dose\# & $\begin{array}{l}\text { ILE } \\
\text { only } \\
\text { used }\end{array}$ & $\begin{array}{l}\text { Other treatments } \\
\text { received, dose } \\
\text { included if } \\
\text { reported }\end{array}$ & ILE effect & Outcome \\
\hline Gallagher, 2010 (33) & \begin{tabular}{|l|} 
Case \\
report
\end{tabular} & $\begin{array}{l}28 \mathrm{y} / \mathrm{M} \\
55.8 \mathrm{~kg}\end{array}$ & \begin{tabular}{|l} 
Lidocaine $2 \%$, \\
Bupivacaine $0.5 \%, 50$ \\
mL mixture LA ratio NR
\end{tabular} & $\begin{array}{l}1.26 \\
2.68\end{array}$ & Subcutaneous & \begin{tabular}{|l|} 
Dizziness then coma \\
Apnea \\
Generalized seizure activity \\
with severe tonic-clonic activity \\
Sudden cardiac arrest
\end{tabular} & $\begin{array}{l}20 \% \\
\text { brand NR }\end{array}$ & $\begin{array}{l}2 \text { units (mL not } \\
\text { reportd) }\end{array}$ & No & \begin{tabular}{|l|} 
Sodium bicarbonate 200 \\
mEq, saline bolus \\
Epinephrine $4 \mathrm{mg}$, \\
vasopressin $40 \mathrm{U}$, atropine 4 \\
mg, midazolam $1 \mathrm{mg}$, \\
lorazepam $2 \mathrm{mg}$ \\
CPR
\end{tabular} & $\begin{array}{l}\text { Resolution of cardiac } \\
\text { symptoms }\end{array}$ & $\begin{array}{l}\text { Survival, } \\
\text { no sequelae }\end{array}$ \\
\hline Gnaho, 2009 (34) & \begin{tabular}{|l|} 
Case \\
report
\end{tabular} & $\begin{array}{l}82 \mathrm{y} / \mathrm{F}, \\
45 \mathrm{~kg}\end{array}$ & Ropivacaine $100 \mathrm{mg}$ & 4.21 & Nerve block & \begin{tabular}{|l|} 
Lost consciousness \\
Generalized tonic-clonic seizure \\
Ventricular fibrillation, no pulse \\
Difficulties in speaking
\end{tabular} & $\begin{array}{l}20 \% \\
\text { Intralipid }\end{array}$ & $\begin{array}{l}70 \mathrm{~mL}(0.31 \mathrm{~g} / \mathrm{kg}) \\
\text { bolus }\end{array}$ & No & $\begin{array}{l}\text { Thiopental } 325 \mathrm{mg}, \\
\text { suxamethonium } 100 \mathrm{mg}, \\
\text { propofol } 30 \mathrm{mg}, \\
\text { epinephrine } 0.3 \mathrm{mg} \\
\text { Oxygen, intubation, CPR } \\
\end{array}$ & \begin{tabular}{|l|} 
Rapid beneficial effect \\
on cardiac resuscitation
\end{tabular} & $\begin{array}{l}\text { Survival, } \\
\text { no sequelae }\end{array}$ \\
\hline Goyal, 2011 (35) & \begin{tabular}{|l|} 
Case \\
report
\end{tabular} & $\begin{array}{l}26 \mathrm{y} / \mathrm{M}, \\
75 \mathrm{~kg}\end{array}$ & \begin{tabular}{|l|} 
Bupivacaine $25 \mathrm{mg}$ \\
\end{tabular} & 2.68 & Nerve block & $\begin{array}{l}\text { Tachycardia }(244-250 / \mathrm{min}) \\
\text { and BP 50-56/30-36 mm }\end{array}$ & \begin{tabular}{|l|}
$10 \%$ \\
Intralipid
\end{tabular} & $\begin{array}{l}150 \mathrm{~mL}(0.20 \mathrm{~g} / \mathrm{kg}) \\
\text { in } 15 \mathrm{~min}\end{array}$ & Yes & NA & $\begin{array}{l}\text { Resolution of cardiac } \\
\text { symptoms }\end{array}$ & \begin{tabular}{|l|} 
Survival, \\
no sequelae
\end{tabular} \\
\hline Grenc, 2011 (36) қ & \begin{tabular}{|l} 
Case \\
report
\end{tabular} & $\begin{array}{l}84 \mathrm{y} / \mathrm{F}, \\
\text { weight NR }\end{array}$ & \begin{tabular}{|l} 
Lidocaine $20 \mathrm{mg}$, \\
Triamcinolone $80 \mathrm{mg}$
\end{tabular} & $\begin{array}{l}1.26 \\
0.92\end{array}$ & Nerve block & $\begin{array}{l}\text { Generalized tonic-clonic } \\
\text { seizures } \\
\text { Cardiac arrest }\end{array}$ & $\begin{array}{l}20 \% \\
\text { Intralipid }\end{array}$ & $100 \mathrm{~mL} \times 2$ bolus & No & $\begin{array}{l}\text { Epinephrine } 2 \mathrm{mg} \text {, atropine } \\
3 \mathrm{mg} \\
\text { Intubation, CPR }\end{array}$ & \begin{tabular}{|l|} 
Resolution of cardiac \\
symptoms; bolus ILE \\
repeated due to \\
persistent hypotension
\end{tabular} & $\begin{array}{l}\text { Survival, } \\
\text { no sequelae }\end{array}$ \\
\hline Hartley, 2012 (37) & \begin{tabular}{|l} 
Case \\
report
\end{tabular} & $\begin{array}{l}46 \mathrm{y} / \mathrm{F}, \\
46 \mathrm{~kg}\end{array}$ & \begin{tabular}{|l} 
Bupivacaine $37.5 \mathrm{mg}+$ \\
$18.75 \mathrm{mg} / \mathrm{hr}$, total dose \\
NR
\end{tabular} & 2.68 & Nerve block & $\begin{array}{l}\text { Coma } \\
\text { Seizures }\end{array}$ & $\begin{array}{l}20 \% \\
\text { Intralipid }\end{array}$ & NR & Yes & Intubation & $\begin{array}{l}\text { Unclear if effect is } \\
\text { related to ILE. }\end{array}$ & $\begin{array}{l}\text { Survival, } \\
\text { sequelae NR }\end{array}$ \\
\hline Harvey, 2011 (38) & \begin{tabular}{|l|} 
Case \\
report
\end{tabular} & $\begin{array}{l}69 \mathrm{y} / \mathrm{F}, \\
80 \mathrm{~kg}\end{array}$ & $\begin{array}{l}\text { Lidocaine } 50 \mathrm{mg}, \\
\text { Bupivacaine } 150 \mathrm{mg}\end{array}$ & 1.26 & Nerve block & $\begin{array}{l}\text { Unresponsiveness, GCS } 3 \\
\text { Seizure } \\
\text { HR 50/min, AV block, BP } \\
51 / 29 \mathrm{mmHg}\end{array}$ & \begin{tabular}{|l|}
$20 \%$ \\
Intralipid
\end{tabular} & $\begin{array}{l}100 \mathrm{~mL}(0.25 \mathrm{~g} / \mathrm{kg}) \\
\text { bolus then } 400 \mathrm{~mL} \\
1.33 \mathrm{~g} / \mathrm{kg} / \mathrm{hr}) \text { in } 45 \\
\mathrm{~min}\end{array}$ & No & $\begin{array}{l}\text { Midazolam } 5 \mathrm{mg} \text {, atropine, } \\
600 \text { mcg, epinephrine } 100 \\
\text { mcg, metaraminol } 4 \mathrm{mg} \\
\text { Intubation, mechanical } \\
\text { ventilation }\end{array}$ & $\begin{array}{l}\text { Resolution of cardiac } \\
\text { symptoms }\end{array}$ & $\begin{array}{l}\text { Survival, } \\
\text { no sequelae }\end{array}$ \\
\hline Heavner, 2012 (39) ฉ & \begin{tabular}{|l|} 
Case \\
report
\end{tabular} & $\begin{array}{l}60 \mathrm{y} / \mathrm{F}, \\
\text { weight NR }\end{array}$ & Lidocaine $1500 \mathrm{mg}$ & 1.26 & Intrapleural & \begin{tabular}{|l} 
Seizure \\
Cardiac arrest
\end{tabular} & \begin{tabular}{|l|}
$20 \%$ \\
brand NR
\end{tabular} & $\begin{array}{l}500 \mathrm{~mL} \text { bolus then } \\
50 \mathrm{~mL} / \mathrm{hr}\end{array}$ & No & $\begin{array}{l}\text { Unspecified conventional } \\
\text { therapy }\end{array}$ & $\begin{array}{l}\text { Resolution of cardiac } \\
\text { symptoms }\end{array}$ & $\begin{array}{l}\text { Survival, } \\
\text { no sequelae }\end{array}$ \\
\hline Hurley 2009 (40) x & \begin{tabular}{|l} 
Case \\
report
\end{tabular} & $\begin{array}{l}54 \mathrm{y} / \mathrm{M}, \\
\text { weight NR }\end{array}$ & Bupivacaine, dose NR & 2.68 & NR & $\begin{array}{l}\text { Cardiac arrest } \\
\text { Asystoly }\end{array}$ & NR & NR & NR & NR & $\begin{array}{l}\text { Resolution of toxicity } \\
\text { within a few minutes }\end{array}$ & $\begin{array}{l}\text { Survival, } \\
\text { no sequelae }\end{array}$ \\
\hline Jensen, 2011 (41) 口 & \begin{tabular}{|l|} 
Case \\
report
\end{tabular} & $\begin{array}{l}41 \mathrm{y} / \mathrm{M}, \\
\text { weight NR }\end{array}$ & Ropivacaine $600 \mathrm{mg}$ & 4.21 & Nerve block & $\begin{array}{l}\text { Loss of consciousness } \\
\text { Seizure }\end{array}$ & \begin{tabular}{|l|}
$20 \%$, \\
Intralipid
\end{tabular} & $100 \mathrm{~mL}$ bolus & No & Diazepam $2.5 \mathrm{mg}$ & $\begin{array}{l}\text { Resolution of } \\
\text { neurologic symptoms }\end{array}$ & \begin{tabular}{|l|} 
Survival, \\
no sequelae
\end{tabular} \\
\hline $\begin{array}{l}\text { Landy, } 2012 \text { (p.463) } \\
\text { (42) }\end{array}$ & \begin{tabular}{|l|} 
Case \\
report
\end{tabular} & $\begin{array}{l}59 \text { y/sex NR, } \\
\text { weight NR }\end{array}$ & Ropivacaine $2250 \mathrm{mg}$ & 4.21 & Nerve block & Seizures & \begin{tabular}{|l|}
$20 \%$, \\
Intralipid
\end{tabular} & $200 \mathrm{~mL}$ bolus & Yes & NR & $\begin{array}{l}\text { Resolution of } \\
\text { neurologic symptoms }\end{array}$ & $\begin{array}{l}\text { Survival, } \\
\text { no sequelae }\end{array}$ \\
\hline \begin{tabular}{|l} 
Landy, 2012 \\
(p.701)(43)
\end{tabular} & \begin{tabular}{|l} 
Case \\
report
\end{tabular} & $\begin{array}{l}74 \mathrm{y} / \mathrm{F}, \\
60 \mathrm{~kg}\end{array}$ & Lidocaine 380 mg & 1.26 & Nerve block & Tonic-clonic movements & \begin{tabular}{|l|}
$20 \%$ \\
Intralipid
\end{tabular} & $\begin{array}{l}200 \mathrm{~mL}(3 \mathrm{~mL} / \mathrm{kg} \\
(0.60 \mathrm{~g} / \mathrm{kg})) \text { bolus }\end{array}$ & No & Flecainide & $\begin{array}{l}\text { Resolution of } \\
\text { symptoms }\end{array}$ & $\begin{array}{l}\text { Survival, } \\
\text { no sequelae }\end{array}$ \\
\hline Lange, 2012 (44) & \begin{tabular}{|l} 
Case \\
report
\end{tabular} & $\begin{array}{l}31 \mathrm{y} / \mathrm{M}, \\
61 \mathrm{~kg}\end{array}$ & Lidocaine $1600 \mathrm{mg}$ & 1.26 & $\begin{array}{l}\text { Subcutaneous/ } \\
\text { Intraperitoneal }\end{array}$ & $\begin{array}{l}\text { Visual hallucinations, } \\
\text { dysarthria, lower level of } \\
\text { consciousness and became } \\
\text { non-verbal }\end{array}$ & $\begin{array}{l}20 \% \\
\text { Intralipid }\end{array}$ & $\begin{array}{l}100 \mathrm{~mL}(0.33 \mathrm{~g} / \mathrm{kg}) \\
\text { in } 10 \mathrm{~min}\end{array}$ & Yes & NR & $\begin{array}{l}\text { Resolution of } \\
\text { neurologic symptoms }\end{array}$ & $\begin{array}{l}\text { Survival, } \\
\text { no sequelae }\end{array}$ \\
\hline
\end{tabular}


AACT manuscript LA to edit for CTX

\begin{tabular}{|c|c|c|c|c|c|c|c|c|c|c|c|c|}
\hline Reference & $\begin{array}{l}\text { Study } \\
\text { type }\end{array}$ & $\begin{array}{l}\text { Age/sex, } \\
\text { weight }\end{array}$ & $\begin{array}{l}\text { Local } \\
\text { anesthetic and } \\
\text { dose }\end{array}$ & $\begin{array}{l}\log D \\
(129)\end{array}$ & $\begin{array}{l}\text { Route of } \\
\text { administration }\end{array}$ & Symptoms & ILE used & ILE dose\# & $\begin{array}{l}\text { ILE } \\
\text { only } \\
\text { used }\end{array}$ & $\begin{array}{l}\text { Other treatments } \\
\text { received, dose } \\
\text { included if } \\
\text { reported }\end{array}$ & ILE effect & Outcome \\
\hline Larson, 2013 (45) & \begin{tabular}{|l|} 
Case \\
report
\end{tabular} & $\begin{array}{l}4 \mathrm{month} / \mathrm{F}, \\
6.54 \mathrm{~kg}\end{array}$ & $\begin{array}{l}\text { Lidocaine } 1500 \mathrm{mg}, \\
\text { Prilocaine } 1500 \mathrm{mg}\end{array}$ & $\begin{array}{l}1.26 \\
1.33\end{array}$ & Topical & \begin{tabular}{|l|} 
Single seizure \\
Tachycardia (147/min) \\
Methemoglobin level was \\
$22.8 \%$
\end{tabular} & $\begin{array}{l}20 \% \\
\text { brand NR }\end{array}$ & $1 \mathrm{~g} / \mathrm{kg}$ bolus & No & $\begin{array}{l}\text { Lorazepam } 0.2 \mathrm{mg} / \mathrm{kg} \text { i.m. } \\
\text { and } 0.2 \mathrm{mg} / \mathrm{kg} \text { i..o., } \\
\text { fosphenytoin } 20 \mathrm{mg} \text { PE } / \mathrm{kg} \text {. } \\
\text { Methylene blue } 10 \mathrm{mg}(1.5 \\
\mathrm{mg} / \mathrm{kg}) \\
\text { Topical decontamination } \\
\text { Intubation, mechanical } \\
\text { ventilation }\end{array}$ & $\begin{array}{l}\text { Unclear if effect is } \\
\text { related to ILE }\end{array}$ & $\begin{array}{l}\text { Survival, } \\
\text { no sequelae }\end{array}$ \\
\hline Levine, 2014(46) & \begin{tabular}{|l|} 
Case \\
report
\end{tabular} & $\begin{array}{l}20 \mathrm{y} / \mathrm{F}, \\
\text { weight NR }\end{array}$ & Bupivacaine, dose NR & 2.68 & Nerve block & Seizure & $\begin{array}{l}20 \%, \\
\text { brand NR }\end{array}$ & $\begin{array}{l}20 \mathrm{~mL} / \mathrm{kg} \text { bolus then } \\
0.25 \mathrm{~mL} / \mathrm{kg} / \mathrm{min} \text { for } \\
3 \text { hours }\end{array}$ & Yes & NR & NR & \begin{tabular}{|l}
$\begin{array}{l}\text { Survival, } \\
\text { increased lipase } 185 \mathrm{IU} / \mathrm{L} \\
\text { suggesting pancreatitis, } \\
\text { resolved after } 14 \text { days }\end{array}$ \\
\end{tabular} \\
\hline Li 2013 (47) 삿 & \begin{tabular}{|l} 
Case \\
report
\end{tabular} & $\begin{array}{l}57 \mathrm{y} / \mathrm{F}, \\
\text { weight NR }\end{array}$ & $\begin{array}{l}\text { Ropivacaine } 75 \mathrm{mg} \text {, } \\
\text { Lidocaine } 400 \mathrm{mg}\end{array}$ & $\begin{array}{l}4.21 \\
1.26\end{array}$ & Nerve block & $\begin{array}{l}\text { Severe pain, somnolent, } \\
\text { pinpoint pupils }\end{array}$ & $\begin{array}{l}\text { Intralipid, } \\
\text { conc. NR }\end{array}$ & $\begin{array}{l}75 \mathrm{~mL} \text { bolus then } \\
\text { infusion, dose and } \\
\text { duration NR } \\
\end{array}$ & No & $\begin{array}{l}\text { Naloxone } 80 \mathrm{mcg}, \\
\text { midazolam } 1 \mathrm{mg} \text {, propofol } \\
30 \mathrm{mg}\end{array}$ & \begin{tabular}{|l}
$\begin{array}{l}\text { Resolution of } \\
\text { symptoms, but } \\
\text { confused and agitated }\end{array}$ \\
\end{tabular} & $\begin{array}{l}\text { Survival, } \\
\text { no sequelae }\end{array}$ \\
\hline Lin, 2010 (48) & \begin{tabular}{|l} 
Case \\
report
\end{tabular} & $\begin{array}{l}2 \text { days } / \mathrm{M}, \\
3.2 \mathrm{~kg}\end{array}$ & Bupivacaine $8 \mathrm{mg}$ & 2.68 & Nerve block & $\begin{array}{l}\text { ST-segment elevation, QRS } \\
\text { widening } \\
\text { Bradycardia }\end{array}$ & $\begin{array}{l}20 \% \\
\text { Intralipid }\end{array}$ & $\begin{array}{l}1 \mathrm{~mL} / \mathrm{kg}(0.2 \mathrm{~g} / \mathrm{kg}) \\
\text { bolus }\end{array}$ & Yes & $\begin{array}{l}\text { No pharmaceuticals } \\
\text { Intubation, CPR }\end{array}$ & $\begin{array}{l}\text { Resolution of cardiac } \\
\text { symptoms }\end{array}$ & $\begin{array}{l}\text { Survival, } \\
\text { no sequelae }\end{array}$ \\
\hline Litz, 2006 (49) & \begin{tabular}{|l} 
Case \\
report
\end{tabular} & $\begin{array}{l}84 \mathrm{y} / \mathrm{F}, \\
50 \mathrm{~kg}\end{array}$ & Ropivacaine $400 \mathrm{mg}$ & 4.21 & Nerve block & $\begin{array}{l}\text { Dizziness, drowsiness } \\
\text { Seizures } \\
\text { Asystole }\end{array}$ & $\begin{array}{l}20 \% \\
\text { Intralipid }\end{array}$ & $\begin{array}{l}100 \mathrm{~mL}(2 \mathrm{~mL} / \mathrm{kg} \\
(0.40 \mathrm{~g} / \mathrm{kg})) \mathrm{bolus}, \\
\text { then } 10 \mathrm{~mL} / \mathrm{min}(2.4 \\
\mathrm{g} / \mathrm{kg} / \mathrm{hr}) \\
\text { Total dose } 200 \mathrm{~mL} \\
(0.8 \mathrm{~g} / \mathrm{kg})\end{array}$ & No & $\begin{array}{l}\text { Thiopental } 150 \mathrm{mg} \text {, } \\
\text { epinephrine } 3 \times 1 \mathrm{mg} \\
\text { Intubation, CPR }\end{array}$ & $\begin{array}{l}\text { Resolution of cardiac } \\
\text { symptoms }\end{array}$ & $\begin{array}{l}\text { Survival, } \\
\text { no sequelae }\end{array}$ \\
\hline Litz, 2008 (50) & \begin{tabular}{|l} 
Case \\
report
\end{tabular} & $\begin{array}{l}91 \mathrm{y} / \mathrm{M}, \\
57 \mathrm{~kg}\end{array}$ & $\begin{array}{l}\text { Mepivacaine } 300 \text { mg, } \\
\text { Prilocaine } 100 \mathrm{mg}\end{array}$ & $\begin{array}{l}1.40 \\
1.33\end{array}$ & Nerve block & $\begin{array}{l}\text { Dizziness, agitation and } \\
\text { developed unresponsiveness } \\
\text { Bigemeny and PVCs }\end{array}$ & $\begin{array}{l}20 \% \\
\text { Intralipid }\end{array}$ & $\begin{array}{l}100 \mathrm{~mL}(0.35 \mathrm{~g} / \mathrm{kg}) \\
\text { bolus then } 0.25 \\
\mathrm{~mL} / \mathrm{kg} / \mathrm{min}(3 \\
\mathrm{g} / \mathrm{kg} / \mathrm{hr}) \\
\text { Total dose } 200 \mathrm{~mL} \\
(0.70 \mathrm{~g} / \mathrm{kg})\end{array}$ & No & Dolastrone $12.5 \mathrm{mg}$ & $\begin{array}{l}\text { Resolution of cardiac } \\
\text { and neurologic } \\
\text { symptoms }\end{array}$ & $\begin{array}{l}\text { Survival, } \\
\text { no sequelae }\end{array}$ \\
\hline Liu, 2012 (51) ฉ & \begin{tabular}{|l|} 
Case \\
report
\end{tabular} & NR & Bupivacaine $200 \mathrm{mg}$ & 2.68 & Intravenous & Unclear symptoms & $\begin{array}{l}\text { Intralipid, } \\
\text { conc. NR }\end{array}$ & \begin{tabular}{|l}
$110 \mathrm{~mL}(1.5 \mathrm{~mL} / \mathrm{kg})$ \\
bolus then 'low \\
dose' infusion in 2 \\
hrs
\end{tabular} & Yes & NR & $\begin{array}{l}\text { Unclear if any } \\
\text { symptoms developed } \\
\text { or were reversed }\end{array}$ & $\begin{array}{l}\text { Survival, } \\
\text { sequelae NR }\end{array}$ \\
\hline Ludot, 2008 (52) & \begin{tabular}{|l|} 
Case \\
report
\end{tabular} & $\begin{array}{l}13 \mathrm{y} / \mathrm{F}, \\
55 \mathrm{~kg} \\
\end{array}$ & \begin{tabular}{|l} 
Lidocaine $200 \mathrm{mg}$, \\
Ropivacaine $150 \mathrm{mg}$ \\
\end{tabular} & $\begin{array}{l}1.26 \\
4.42 \\
\end{array}$ & Nerve block & $\begin{array}{l}\text { Ventricular tachycardia with } \\
\text { wide QRS }\end{array}$ & $\begin{array}{l}20 \%, \\
\text { Medialipid }\end{array}$ & $\begin{array}{l}150 \mathrm{~mL}(3 \mathrm{~mL} / \mathrm{kg} \\
(0.60 \mathrm{~g} / \mathrm{kg})) \text { bolus } \\
\end{array}$ & Yes & $\begin{array}{l}\begin{array}{l}\text { No pharmaceuticals } \\
\text { Manual ventilation }\end{array} \\
\end{array}$ & $\begin{array}{l}\begin{array}{l}\text { Resolution of cardiac } \\
\text { symptoms }\end{array} \\
\end{array}$ & $\begin{array}{l}\begin{array}{l}\text { Survival, } \\
\text { no sequelae }\end{array} \\
\end{array}$ \\
\hline Markowitz, 2009 (53) & \begin{tabular}{|l|} 
Case \\
report
\end{tabular} & $\begin{array}{l}17 \mathrm{y} / \mathrm{M}, \\
61 \mathrm{~kg}\end{array}$ & Bupivacaine $100 \mathrm{mg}$ & 2.68 & Nerve block & \begin{tabular}{|l} 
Coma \\
Status epilepticus \\
Ventricular fibrillation
\end{tabular} & $\begin{array}{l}20 \% \\
\text { Intralipid }\end{array}$ & $\begin{array}{l}500 \mathrm{~mL}(8 \mathrm{ml} / \mathrm{kg} \\
(1.6 \mathrm{~g} / \mathrm{kg})) \text {, dose } \\
\text { regimen NR }\end{array}$ & No & $\begin{array}{l}\text { Midazolam } 3 \mathrm{mg} \\
\text { Intubation }\end{array}$ & $\begin{array}{l}\text { Unclear if effect is } \\
\text { related to ILE }\end{array}$ & $\begin{array}{l}\text { Survival, } \\
\text { no sequelae }\end{array}$ \\
\hline
\end{tabular}


AACT manuscript LA to edit for CTX

\begin{tabular}{|c|c|c|c|c|c|c|c|c|c|c|c|c|}
\hline Reference & \begin{tabular}{|l} 
Study \\
type
\end{tabular} & $\begin{array}{l}\text { Age/sex, } \\
\text { weight }\end{array}$ & $\begin{array}{l}\text { Local } \\
\text { anesthetic and } \\
\text { dose }\end{array}$ & $\left|\begin{array}{l}\log D \\
(129)\end{array}\right|$ & $\begin{array}{l}\text { Route of } \\
\text { administration }\end{array}$ & Symptoms & ILE used & ILE dose\# & $\begin{array}{l}\text { ILE } \\
\text { only } \\
\text { used }\end{array}$ & $\begin{array}{l}\text { Other treatments } \\
\text { received, dose } \\
\text { included if } \\
\text { reported }\end{array}$ & ILE effect & Outcome \\
\hline Marraffa, 2013 (54) 凤 & \begin{tabular}{|l|} 
Case \\
report
\end{tabular} & $\begin{array}{l}\begin{array}{l}66 \mathrm{y} / \mathrm{F}, \\
\text { weight NR }\end{array} \\
\end{array}$ & Bupivacaine $420 \mathrm{mg}$ & 2.68 & Subcutaneous & $\begin{array}{l}\text { CNS depression, declining } \\
\text { mental status } \\
\text { Generalize tonic-clonic seizure } \\
\text { activity } \\
\text { Systolic hypotension to } 60 \\
\text { mmHg }\end{array}$ & $\begin{array}{l}20 \%, \\
\text { brand NR }\end{array}$ & $500 \mathrm{~mL} \times 2$ bolus & No & $\begin{array}{l}\text { Hydromorphone } 60 \mathrm{mg} \text { with } \\
\text { the LA. Bicarbonate } \\
\text { empirically given, } \\
\text { dopamine. Naloxone } 0.4 \mathrm{mg} \\
\text { x2, icepacks were applied } \\
\text { every } 2 \mathrm{hr} \text { at the injection } \\
\text { site, lorazepam } 0.2 \mathrm{mg} \\
\text { Intubation }\end{array}$ & $\begin{array}{l}\text { Resolution of cardiac } \\
\text { symptoms }\end{array}$ & $\begin{array}{l}\text { Survival, } \\
\text { no sequelae }\end{array}$ \\
\hline Marwick, 2009 (55) & \begin{tabular}{|l|} 
Case \\
report
\end{tabular} & $\begin{array}{l}33 \mathrm{y} / \mathrm{M}, \\
72 \mathrm{~kg}\end{array}$ & Bupivacaine $112.5 \mathrm{mg}$ & 2.68 & Nerve block & \begin{tabular}{|l} 
Seizure \\
Wide QRS \\
Cardiac arrest \\
Dry mouth, apnea
\end{tabular} & \begin{tabular}{|l|}
$20 \%$ \\
Intralipid
\end{tabular} & $\begin{array}{l}150 \mathrm{~mL}(0.43 \mathrm{~g} / \mathrm{kg}) \\
\text { bolus then } 350 \mathrm{~mL} \\
(1.94 \mathrm{~g} / \mathrm{kg} / \mathrm{hr}) \text { in } 30 \\
\mathrm{~min}\end{array}$ & No & \begin{tabular}{|l|} 
Epinephrine $1 \mathrm{mg}+0.06$ \\
$\mathrm{mcg} / \mathrm{kg} / \mathrm{min}$ infusion, total \\
time NR. Thiopental $250 \mathrm{mg}$, \\
sodium bicarbonate, insulin, \\
potassium, amiodarone 300 \\
$\mathrm{mg}$ in $30 \mathrm{~min}$ \\
Oxygen, intubation, CPR
\end{tabular} & $\begin{array}{l}\text { Resolution of cardiac } \\
\text { symptoms }\end{array}$ & \begin{tabular}{|l|} 
Survival, \\
amylase $608 \mathrm{IU} / \mathrm{L}$
\end{tabular} \\
\hline Mazoit, 2013 (56) & \begin{tabular}{|l|} 
Case \\
report
\end{tabular} & $\begin{array}{l}4 \mathrm{y} / \mathrm{M}, \\
\text { weight NR }\end{array}$ & Ropivacaine $260 \mathrm{mg}$ & 4.21 & Nerve block & $\begin{array}{l}\text { Metallic taste, myoclonic } \\
\text { movement } \\
\text { Seizure } \\
\text { Cardiac arrest with asystole }\end{array}$ & \begin{tabular}{|l|}
$20 \%$ \\
Intralipid
\end{tabular} & $100 \mathrm{~mL}$ bolus & No & \begin{tabular}{|l|} 
Epinephrine $100 \mathrm{mcg}$ \\
Manual ventilation, CPR
\end{tabular} & $\begin{array}{l}\text { Resolution of cardiac } \\
\text { symptoms }\end{array}$ & \begin{tabular}{|l|} 
Survival, \\
no sequelae
\end{tabular} \\
\hline $\begin{array}{l}\begin{array}{l}\text { Mccutchen, } 2008 \\
\text { (57) }\end{array} \\
\end{array}$ & \begin{tabular}{|l|} 
Case \\
report
\end{tabular} & $\begin{array}{l}82 \mathrm{y} / \mathrm{F}, \\
\text { weight NR }\end{array}$ & Bupivacaine $150 \mathrm{mg}$ & 2.68 & Nerve block & $\begin{array}{l}\text { Seizures } \times 2 \\
\text { VT at } 200 / \mathrm{min}\end{array}$ & $\begin{array}{l}20 \% \\
\text { Intralipid }\end{array}$ & $\begin{array}{l}100 \mathrm{~mL} \text { bolus then } \\
400 \mathrm{~mL} \text { over } 15 \mathrm{~min}\end{array}$ & No & \begin{tabular}{|l|} 
Midazolam $3 \mathrm{mg}$, \\
amiodarone $150 \mathrm{mg}$, \\
unspecified ACLL drugs \\
Oxygen, defibrillation
\end{tabular} & $\begin{array}{l}\text { Unclear if effect is } \\
\text { related to ILE }\end{array}$ & $\begin{array}{l}\text { Survival, } \\
\text { no sequelae }\end{array}$ \\
\hline Mizutani, 2011 (58) & \begin{tabular}{|l|} 
Case \\
report
\end{tabular} & $\begin{array}{l}24 \mathrm{y} / \mathrm{M}, \\
66 \mathrm{~kg}\end{array}$ & Ropivacaine $200 \mathrm{mg}$ & 4.21 & Nerve block & $\begin{array}{l}\text { Disappearance of motor } \\
\text { response to stimulation }\end{array}$ & $\begin{array}{l}20 \% \\
\text { brand NR }\end{array}$ & $\begin{array}{l}100 \mathrm{~mL}(0.30 \mathrm{~g} / \mathrm{kg}) \\
\text { bolus }\end{array}$ & No & \begin{tabular}{|l|} 
Propofol (titrated), fentanyl \\
$100 \mu \mathrm{g}$ \\
Mechanical ventilation \\
during general anesthesia
\end{tabular} & $\begin{array}{l}\begin{array}{l}\text { Resolution of } \\
\text { neurologic symptoms, } \\
\text { but unclear if effect is } \\
\text { related to ILE. }\end{array} \\
\end{array}$ & $\begin{array}{l}\text { Survival, } \\
\text { no sequelae }\end{array}$ \\
\hline Nguyen, 2012 (59) & \begin{tabular}{|l} 
Case \\
report
\end{tabular} & $\begin{array}{l}19 \mathrm{y} / \mathrm{M}, \\
72 \mathrm{~kg}\end{array}$ & Ropivacaine 75 mg & 4.21 & Nerve block & $\begin{array}{l}\text { Visual hallucinations } \\
\text { Sinus tachycardia and } \\
\text { hypertension } \\
\text { Myoclonic movements of the } \\
\text { head and neck }\end{array}$ & $\begin{array}{l}20 \% \\
\text { Intralipid }\end{array}$ & \begin{tabular}{|l}
$100 \mathrm{~mL}(0.28 \mathrm{~g} / \mathrm{kg})$ \\
bolus
\end{tabular} & No & $\begin{array}{l}\text { Midazolam } 2 \mathrm{mg} \text { x2 } \\
\text { Oxygen }\end{array}$ & $\begin{array}{l}\text { Resolution of } \\
\text { neurologic symptoms }\end{array}$ & $\begin{array}{l}\text { Survival, } \\
\text { no sequelae }\end{array}$ \\
\hline Ogugua, 2009(60) ฉ & \begin{tabular}{|l|} 
Case \\
report
\end{tabular} & $\begin{array}{l}47 \mathrm{y} / \mathrm{F}, \\
\text { weight NR }\end{array}$ & Bupivacaine $165 \mathrm{mg}$ & 2.68 & Nerve block & \begin{tabular}{|l} 
Seizure \\
Asystole
\end{tabular} & $\begin{array}{l}20 \%, \\
\text { brand NR }\end{array}$ & $\begin{array}{l}160 \mathrm{~mL} \text { bolus then } \\
200 \mathrm{~mL} \text { infusion, } \\
\text { duration NR }\end{array}$ & No & $\begin{array}{l}\text { Midazolam } 2 \mathrm{mg} \text {, } \\
\text { epinephrine } 9 \mathrm{mg} \text {, ACLS } \\
\text { protocol to ROSC } \\
\text { Intubation }\end{array}$ & $\begin{array}{l}\text { Apparent improvement } \\
\text { in cardiac output }\end{array}$ & $\begin{array}{l}\text { Survival, } \\
\text { no sequelae }\end{array}$ \\
\hline Reddy, 2010 (61) ฉ & \begin{tabular}{|l|} 
Case \\
report
\end{tabular} & $\begin{array}{l}59 \mathrm{y} / \mathrm{M}, \\
\text { weight NR }\end{array}$ & \begin{tabular}{|l} 
Mepivacaine, \\
Ropivacaine \\
$50 \mathrm{~mL} 50 / 50$ mixture, \\
conc. NR
\end{tabular} & $\begin{array}{l}1.40 \\
4.21\end{array}$ & Nerve block & \begin{tabular}{|l|} 
Agitation \\
Seizures \\
Tachycardia $(160-170 / \mathrm{min})$ \\
Slurred speech
\end{tabular} & \begin{tabular}{|l|}
$20 \%$ \\
Intralipid
\end{tabular} & $\begin{array}{l}1.5 \mathrm{~mL} / \mathrm{kg} \text { bolus } \\
\text { then } 0.25 \\
\mathrm{~mL} / \mathrm{kg} / \mathrm{min} \text { in } 60 \\
\mathrm{~min}\end{array}$ & Yes & $\begin{array}{l}\text { No pharmaceuticals } \\
\text { Oxygen }\end{array}$ & $\begin{array}{l}\text { Resolution of cardiac } \\
\text { and neurologic } \\
\text { symptoms }\end{array}$ & $\begin{array}{l}\text { Survival, } \\
\text { no sequelae }\end{array}$ \\
\hline
\end{tabular}


AACT manuscript LA to edit for CTX

\begin{tabular}{|c|c|c|c|c|c|c|c|c|c|c|c|c|}
\hline Reference & $\begin{array}{l}\text { Study } \\
\text { type }\end{array}$ & $\begin{array}{l}\text { Age/sex, } \\
\text { weight }\end{array}$ & $\begin{array}{l}\text { Local } \\
\text { anesthetic and } \\
\text { dose }\end{array}$ & $\begin{array}{l}\log D \\
(129)\end{array}$ & $\begin{array}{l}\text { Route of } \\
\text { administration }\end{array}$ & Symptoms & ILE used & ILE dose\# & 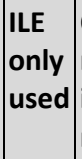 & $\begin{array}{l}\text { Other treatments } \\
\text { received, dose } \\
\text { included if } \\
\text { reported }\end{array}$ & ILE effect & Outcome \\
\hline Rosenblatt, 2006 (1) & \begin{tabular}{|l|} 
Case \\
report
\end{tabular} & $\begin{array}{l}58 \mathrm{y} / \mathrm{M}, \\
82 \mathrm{~kg}\end{array}$ & $\begin{array}{l}\text { Bupivacaine } 100 \mathrm{mg}, \\
\text { Mepivacaine } 300 \mathrm{mg}\end{array}$ & $\begin{array}{l}2.68 \\
1.40\end{array}$ & Nerve block & $\begin{array}{l}\text { Incoherent } \\
\text { Repeated seizures } \\
\text { Apneic } \\
\text { Asystole }\end{array}$ & \begin{tabular}{|l}
$20 \%$ \\
Intralipid
\end{tabular} & $\begin{array}{l}100 \mathrm{~mL}(0.24 \mathrm{~g} / \mathrm{kg}) \\
\text { bolus then } 0.5 \\
\mathrm{~mL} / \mathrm{kg} / \mathrm{min}(6.0 \\
\mathrm{g} / \mathrm{kg} / \mathrm{hr}) \text { in } 60 \mathrm{~min}\end{array}$ & No & \begin{tabular}{|l|} 
Epinephrine $3 \mathrm{mg}$, atropine \\
$2 \mathrm{mg}$, arginine vasopressin \\
$40 \mathrm{U}$, amiodarone $300 \mathrm{mg}$, \\
propofol $150 \mathrm{mg}$ \\
Mechanical ventilation, CPR, \\
defibrillation
\end{tabular} & $\begin{array}{l}\text { Resolution of cardiac } \\
\text { symptoms }\end{array}$ & \begin{tabular}{|l|} 
Survival, \\
no sequelae
\end{tabular} \\
\hline Sakai, 2010 (62) & \begin{tabular}{|l|} 
Case \\
report
\end{tabular} & $\begin{array}{l}0 \mathrm{y} / \mathrm{F}, \\
40 \mathrm{~kg}\end{array}$ & Ropivacaine $150 \mathrm{mg}$ & 4.21 & Nerve block & \begin{tabular}{|l|} 
Lowered responsiveness, \\
paleness, peripheral coldness, \\
restlessness, hypotension, \\
shallow irregular breathing, \\
clonic convulsions in the limbs
\end{tabular} & \begin{tabular}{|l|}
$20 \%$ \\
Intralipos
\end{tabular} & $\begin{array}{l}5 \times 10 \mathrm{ml}(0.25 \mathrm{~g} / \mathrm{kg}) \\
\text { bolus, then } 100 \mathrm{ml} \\
(0.5 \mathrm{~g} / \mathrm{kg}) \text { in } 50 \\
\mathrm{~min} \text {., then } 20 \mathrm{ml} / \mathrm{hr} \\
(0.1 \mathrm{~g} / \mathrm{kg} / \mathrm{hr}) \text {. Total } \\
\text { dose } 230 \mathrm{ml}\end{array}$ & No & $\begin{array}{l}\text { Etilefrine (dose NR), } \\
\text { diazepam } 5 \mathrm{mg}\end{array}$ & \begin{tabular}{|l}
$\begin{array}{l}\text { Resolution of } \\
\text { symptoms }\end{array}$ \\
\end{tabular} & \begin{tabular}{|l|} 
Survival, \\
no sequelae
\end{tabular} \\
\hline Schaeffer, 2010 (63) & \begin{tabular}{|l|} 
Case \\
report
\end{tabular} & $\begin{array}{l}74 \mathrm{y} / \mathrm{F}, \\
60 \mathrm{~kg}\end{array}$ & Lidocaine $400 \mathrm{mg}$ & 1.26 & Nerve block & $\begin{array}{l}\text { Confused, disoriented, had loss } \\
\text { of consciousness and } \\
\text { myoclonus of the face }\end{array}$ & \begin{tabular}{|l|}
$20 \%$ \\
Intralipid
\end{tabular} & $\begin{array}{l}200 \mathrm{~mL}(0.67 \mathrm{~g} / \mathrm{kg}) \\
\text { bolus }\end{array}$ & Yes & NR & \begin{tabular}{|l|} 
Apparent improvement \\
of symptoms, but \\
unclear if effect is \\
related to ILE
\end{tabular} & $\begin{array}{l}\text { Survival, } \\
\text { no sequelae }\end{array}$ \\
\hline $\begin{array}{l}\text { Schellhammer } 2011 \\
\text { (64) }\end{array}$ & \begin{tabular}{|l} 
Case \\
report
\end{tabular} & $\begin{array}{l}54 \mathrm{y} / \mathrm{F}, \\
\text { weight NR }\end{array}$ & Mepivacaine 1000 mg & 1.40 & Nerve block & \begin{tabular}{|l|} 
Dysphagia, dyspnea, PVC with \\
bigeminy, ventricular \\
tachycardia 145 bpm, perioral \\
automatisms, dysarthria, \\
hallucinations, progressive loss \\
of consciousness and finally \\
seizure
\end{tabular} & $\begin{array}{l}20 \%, \\
\text { Lipofundin }\end{array}$ & $\begin{array}{l}\text { Infusion, specific } \\
\text { dose and duration } \\
\text { NR }\end{array}$ & No & $\begin{array}{l}\text { Amiodarone } 5 \mathrm{mg} / \mathrm{kg} \text {, } \\
\text { midazolam, propofol } \\
\text { Oxygen }\end{array}$ & $\begin{array}{l}\text { Transient improvement } \\
\text { in level of } \\
\text { consciousness }\end{array}$ & $\begin{array}{l}\text { Survival, } \\
\text { no sequelae }\end{array}$ \\
\hline Scherrer $2013(65) \not$ & \begin{tabular}{|l} 
Case \\
report
\end{tabular} & $\begin{array}{l}25 \mathrm{y} / \mathrm{F}, \\
\text { weight NR }\end{array}$ & Ropivacine $450 \mathrm{mg}$ & 4.21 & $\begin{array}{l}\text { Intraperitoneal/ nerve } \\
\text { block }\end{array}$ & Seizure, ventricular arrhythmia & $\begin{array}{l}20 \% \\
\text { brand NR }\end{array}$ & $\begin{array}{l}\text { Infusion, specific } \\
\text { dose and duration } \\
\text { NR }\end{array}$ & Yes & NR & $\begin{array}{l}\text { Ventricular arrhythmia } \\
\text { converted to sinus } \\
\text { rhythm }\end{array}$ & $\begin{array}{l}\text { Survival, } \\
\text { sequelae NR }\end{array}$ \\
\hline $\begin{array}{l}\text { Schwarzkopf, } 2011 \\
\text { (66) ฉ }\end{array}$ & \begin{tabular}{|l|} 
Case \\
report
\end{tabular} & NR & $\begin{array}{l}\text { Prilocaine } 300 \mathrm{mg}, \\
\text { Bupivacaine } 50 \mathrm{mg}\end{array}$ & $\begin{array}{l}1.33 \\
2.68\end{array}$ & Nerve block & \begin{tabular}{|l} 
Seizures \\
Hypertension
\end{tabular} & $\begin{array}{l}20 \% \\
\text { brand NR }\end{array}$ & $\begin{array}{l}1.5 \mathrm{~mL} / \mathrm{kg} \text { bolus } \\
\text { then } 0.1 \mathrm{~mL} / \mathrm{kg} \text { in } \\
30 \mathrm{~min}\end{array}$ & No & $\begin{array}{l}\text { Midazolam } 10 \mathrm{mg} \\
\text { Manual ventilation }\end{array}$ & $\begin{array}{l}\text { Unclear if effect is } \\
\text { related to ILE }\end{array}$ & \begin{tabular}{|l|} 
Survival, \\
sequelae NR
\end{tabular} \\
\hline Shah, 2009 (67) & \begin{tabular}{|l|} 
Case \\
report
\end{tabular} & $\begin{array}{l}40 \text { days } / \mathrm{M}, \\
4.96 \mathrm{~kg}\end{array}$ & Bupivacaine $10 \mathrm{mg}$ & 2.68 & Nerve block & \begin{tabular}{l|} 
BP $31 / 19 \mathrm{mmHg}$; tachycardia \\
(170/min); The ST segment was \\
noted to be elevated $2-3 \mathrm{~mm}$ \\
and the T-wave was inverted
\end{tabular} & \begin{tabular}{|l|}
$20 \%$ \\
Intralipid
\end{tabular} & $\begin{array}{l}10 \mathrm{~mL}(2 \mathrm{~mL} / \mathrm{kg}(0.4 \\
\mathrm{g} / \mathrm{kg})) \text { bolus }\end{array}$ & No & $\begin{array}{l}\text { Epinephrine } 2 \mathrm{mcg} / \mathrm{kg} \times 2 \text {, } \\
\text { albumin } 5 \% 20 \mathrm{~mL} \text {. } \\
\text { Mechanical ventilation } \\
\text { during general anesthesia }\end{array}$ & $\begin{array}{l}\text { Resolution of cardiac } \\
\text { symptoms }\end{array}$ & \begin{tabular}{|l|} 
Survival, \\
no sequelae
\end{tabular} \\
\hline Shenoy, 2014 (68) & \begin{tabular}{|l} 
Case \\
report
\end{tabular} & $\begin{array}{l}3 \mathrm{y} / \mathrm{sex} \mathrm{NR}, \\
11 \mathrm{~kg}\end{array}$ & Bupivacaine $25 \mathrm{mg}$ & 2.68 & Nerve block & $\begin{array}{l}\text { Pulseless ventricular } \\
\text { tachycardia }\end{array}$ & $\begin{array}{l}20 \% \\
\text { brand NR }\end{array}$ & $\begin{array}{l}15 \mathrm{~mL}(0.27 \mathrm{~g} / \mathrm{kg}) \\
\text { bolus then } 150 \\
\mathrm{~mL} / \mathrm{hr}(2.73 \\
\mathrm{g} / \mathrm{kg} / \mathrm{hr}) \text { in } 15 \mathrm{~min}, \\
\text { then } 5 \mathrm{~mL}(0.091 \\
\mathrm{g} / \mathrm{kg} \text { ) bolus. Total } \\
\text { dose } 170 \mathrm{~mL}(3.1 \\
\mathrm{g} / \mathrm{kg})\end{array}$ & No & $\begin{array}{l}\text { Epinephrine } 0.03 \mathrm{mg} \\
\text { Oxygen, CPR }\end{array}$ & \begin{tabular}{|l|} 
Beneficial effect with \\
resolution of cardiac \\
symptoms together \\
with other treatments.
\end{tabular} & $\begin{array}{l}\text { Survival, } \\
\text { no sequelae }\end{array}$ \\
\hline
\end{tabular}


AACT manuscript LA to edit for CTX

\begin{tabular}{|c|c|c|c|c|c|c|c|c|c|c|c|c|}
\hline Reference & $\begin{array}{l}\text { Study } \\
\text { type }\end{array}$ & $\begin{array}{l}\text { Age/sex, } \\
\text { weight }\end{array}$ & $\begin{array}{l}\text { Local } \\
\text { anesthetic and } \\
\text { dose }\end{array}$ & $\left|\begin{array}{l}\log D \\
(129)\end{array}\right|$ & $\begin{array}{l}\text { Route of } \\
\text { administration }\end{array}$ & Symptoms & ILE used & ILE dose\# & $\begin{array}{l}\text { ILE } \\
\text { only } \\
\text { used }\end{array}$ & $\begin{array}{l}\text { Other treatments } \\
\text { received, dose } \\
\text { included if } \\
\text { reported }\end{array}$ & ILE effect & Outcome \\
\hline Shih, 2011 (69) & \begin{tabular}{|l|} 
Case \\
report
\end{tabular} & $\begin{array}{l}69 \mathrm{y} / \mathrm{F}, \\
48.5 \mathrm{~kg}\end{array}$ & \begin{tabular}{|l|} 
Lidocaine $225 \mathrm{mg}$, \\
Bupivacaine $37.5 \mathrm{mg}$
\end{tabular} & $\begin{array}{l}1.26 \\
2.68\end{array}$ & Nerve block & $\begin{array}{l}\text { Bradycardia, reduced blood } \\
\text { pressure } \\
\text { Obtuned, unable to fully } \\
\text { arouse }\end{array}$ & \begin{tabular}{|l|}
$20 \%$, \\
Lipovenoes
\end{tabular} & \begin{tabular}{|l|}
$50 \mathrm{~mL}(0.21 \mathrm{~g} / \mathrm{kg})$ \\
bolus
\end{tabular} & No & $\begin{array}{l}\text { Atropine } 0.5 \mathrm{mg} \times 3, \\
\text { ephedrine } 10 \mathrm{mg}\end{array}$ & $\begin{array}{l}\text { Resolution of cardiac } \\
\text { and neurologic } \\
\text { symptoms }\end{array}$ & \begin{tabular}{|l|} 
Survival, \\
no sequelae
\end{tabular} \\
\hline Smith, 2008 (70) & \begin{tabular}{|l|} 
Case \\
report
\end{tabular} & $\begin{array}{l}83 \mathrm{y} / \mathrm{M}, \\
75 \mathrm{~kg}\end{array}$ & Bupivacaine $130 \mathrm{mg}$ & 2.68 & Nerve block & \begin{tabular}{|l|} 
Loss of consciousness \\
Seizure \\
Pulseless wide complex \\
tachycardia and asystole
\end{tabular} & $\begin{array}{l}20 \%, \\
\text { brand NR }\end{array}$ & \begin{tabular}{|l|}
$250 \mathrm{~mL}(3 \mathrm{~mL} / \mathrm{kg}$ \\
$(0.60 \mathrm{~g} / \mathrm{kg}))$ bolus \\
then $0.2 \mathrm{~mL} / \mathrm{kg} / \mathrm{min}$ \\
$(2.4 \mathrm{~g} / \mathrm{kg} / \mathrm{hr})$
\end{tabular} & No & $\begin{array}{l}\text { Epinephrine } 1 \mathrm{mg} \text {, atropine } \\
1 \mathrm{mg} \text { (dosed after lipid } \\
\text { emulsion), midazolam } 2 \mathrm{mg} . \\
\text { Oxygen, manual ventilation, } \\
\text { CPR; then intubation, } \\
\text { mechanical ventilation }\end{array}$ & \begin{tabular}{|l|} 
Resolution of cardiac \\
symptoms, but unclear \\
of effect is related to \\
ILE
\end{tabular} & \begin{tabular}{|l|} 
Survival, \\
no sequelae
\end{tabular} \\
\hline Sonsino, 2009 (71) & \begin{tabular}{|l|} 
Case \\
report
\end{tabular} & $\begin{array}{l}2 \mathrm{y} / \mathrm{F}, \\
\text { weight NR }\end{array}$ & \begin{tabular}{|l|} 
Ropivacaine $150 \mathrm{mg}$ \\
\end{tabular} & 4.21 & Nerve block & $\begin{array}{l}\text { Generalized tonic-clonic seizure } \\
\times 1 \\
\text { Asystole }\end{array}$ & $\begin{array}{l}\text { Kabiven } \\
2000, \\
\text { conc. NR }\end{array}$ & $50 \mathrm{~mL}$ bolus & No & $\begin{array}{l}\text { Propofol } 30 \mathrm{mg}, \\
\text { epinephrine } 0.3 \mathrm{mg} \text { (ACLS). } \\
\text { Intubation, mechanical } \\
\text { ventilation }\end{array}$ & $\begin{array}{l}\text { Resolution of cardiac } \\
\text { symptoms }\end{array}$ & \begin{tabular}{|l|} 
Survival, \\
no sequelae (died from \\
bronchopneumonia 10 days \\
after)
\end{tabular} \\
\hline Sorrenti 2014 (72) ฉ & \begin{tabular}{|l} 
Case \\
report
\end{tabular} & $\begin{array}{l}46 \mathrm{y} / \mathrm{M}, \\
\text { weight NR }\end{array}$ & Mepivacaine $360 \mathrm{mg}$ & 1.40 & Nerve block & $\begin{array}{l}\text { Dysarthria, confusion, loss of } \\
\text { verbal contact, agitation, } \\
\text { tachycardia, hypertension }\end{array}$ & $\begin{array}{l}20 \% \\
\text { Intralipid }\end{array}$ & $\begin{array}{l}150 \mathrm{~mL} \text { bolus then } \\
0.25 \mathrm{~mL} / \mathrm{kg} / \mathrm{min} . \\
\text { Total dose } 250 \mathrm{~mL}\end{array}$ & No & Midazolam $2.5 \mathrm{mg}$ & $\begin{array}{l}\text { Resolution of } \\
\text { neurological and } \\
\text { cardiac symptoms }\end{array}$ & $\begin{array}{l}\text { Survival, } \\
\text { no sequelae }\end{array}$ \\
\hline Spence, 2007 (73) & \begin{tabular}{|l|} 
Case \\
report
\end{tabular} & $\begin{array}{l}18 \mathrm{y} / \mathrm{F}, 38 \mathrm{w} \\
\text { pregnant, } \\
86 \mathrm{~kg}\end{array}$ & \begin{tabular}{|l|} 
Lidocaine $80 \mathrm{mg}$, \\
Bupivacaine $65 \mathrm{mg}$
\end{tabular} & $\begin{array}{l}1.26 \\
2.68\end{array}$ & Nerve block & $\begin{array}{l}\text { Restless, agitated, did not obey } \\
\text { commands, unresponsive. Fetal } \\
\text { heart rate decelerating }\end{array}$ & $\begin{array}{l}20 \% \\
\text { Intralipid }\end{array}$ & \begin{tabular}{|l|}
$50 \mathrm{~mL}(0.12 \mathrm{~g} / \mathrm{kg}) \times 2$ \\
bolus
\end{tabular} & No & $\begin{array}{l}\text { General anesthesia for } \\
\text { delivery } \\
\text { Neonatal intubation }\end{array}$ & \begin{tabular}{|l|} 
Resolution of \\
neurologic symptoms
\end{tabular} & $\begin{array}{l}\text { Survival, } \\
\text { no sequelae }\end{array}$ \\
\hline Sturini, $2010(74) \not$ & \begin{tabular}{|l|} 
Case \\
report
\end{tabular} & NR & Mepivacaine $750 \mathrm{mg}$ & 1.40 & Intravenous & $\begin{array}{l}\text { Numbness, light headedness } \\
\text { dizziness slurred speech }\end{array}$ & $\begin{array}{l}20 \% \\
\text { Intralipid }\end{array}$ & $100 \mathrm{~mL}$ bolus & Yes & NR & \begin{tabular}{|l|}
$\begin{array}{l}\text { Possibly prevented } \\
\text { cardiac symptoms from } \\
\text { LA toxicity }\end{array}$ \\
\end{tabular} & $\begin{array}{l}\text { Survival, } \\
\text { no sequelae }\end{array}$ \\
\hline Süzer 2011 (75) & \begin{tabular}{|l} 
Case \\
report
\end{tabular} & $\begin{array}{l}71 \mathrm{y} / \mathrm{M}, \\
78 \mathrm{~kg}\end{array}$ & $\begin{array}{l}\text { Bupivacaine } 50 \mathrm{mg} \text {, } \\
\text { Lidocaine } 200 \mathrm{mg}\end{array}$ & $\begin{array}{l}2.68 \\
1.26\end{array}$ & Nerve block & $\begin{array}{l}\text { Loss of consciousness, } \\
\text { dyspnea, hypotension 65/40 } \\
\text { mmHg, ventricular } \\
\text { extrasystoles, tachycardia } 140 \\
\text { bpm, seizures }\end{array}$ & $\begin{array}{l}20 \% \\
\text { Intralipid }\end{array}$ & $\begin{array}{l}0.5 \mathrm{~mL} / \mathrm{kg} / \mathrm{min}(6.0 \\
\mathrm{g} / \mathrm{kg} / \mathrm{hr} \text { ) infusion. } \\
\text { Total dose } 500 \mathrm{~mL} \\
(1.3 \mathrm{~g} / \mathrm{kg})\end{array}$ & No & $\begin{array}{l}\text { Midazolam } 5 \mathrm{mg}, \\
\text { epinephrine } 10 \mathrm{mg}, \\
\text { amiodarone } 150 \mathrm{mg} \\
\text { Intubation }\end{array}$ & \begin{tabular}{|l|} 
Resolution of cardiac \\
symptoms within 3 \\
min, resolution of \\
neurological symptoms \\
within total dose \\
administered
\end{tabular} & $\begin{array}{l}\text { Survival, } \\
\text { no sequelae }\end{array}$ \\
\hline Ter Horst, 2010 (76) & \begin{tabular}{|l|} 
Case \\
report
\end{tabular} & $\begin{array}{l}27 \mathrm{y} / \mathrm{F}, \\
\text { weight NR }\end{array}$ & Ropivacaine $300 \mathrm{mg}$ & 4.21 & Nerve block & $\begin{array}{l}\text { Decreased level of } \\
\text { consciousness } \\
\text { Seizure }\end{array}$ & $\begin{array}{l}20 \% \\
\text { Intralipid }\end{array}$ & $\begin{array}{l}100 \mathrm{~mL}(1.5 \mathrm{~mL} / \mathrm{kg}) \\
\text { bolus then } 400 \mathrm{~mL} \\
\text { in } 1.5 \text { hrs }\end{array}$ & No & $\begin{array}{l}\text { Midazolam } 5 \mathrm{mg} \times 2 . \\
\text { Mechanical ventilation until } \\
\text { resolution of respiratory } \\
\text { symptoms }\end{array}$ & \begin{tabular}{|l|} 
Rapid beneficial effect \\
on neurologic \\
symptoms
\end{tabular} & $\begin{array}{l}\text { Survival, } \\
\text { no sequelae }\end{array}$ \\
\hline Varela, 2010 (77) & \begin{tabular}{|l|} 
Case \\
report
\end{tabular} & $\begin{array}{l}83 \mathrm{y} / \mathrm{F}, \\
70 \mathrm{~kg}\end{array}$ & $\begin{array}{l}\text { Bupivacaine } 150 \mathrm{mg}, \\
\text { Ropivacaine } 300 \mathrm{mg}\end{array}$ & $\begin{array}{l}2.68 \\
4.21\end{array}$ & Nerve block & $\begin{array}{l}\text { Repeated seizures. } \\
\text { Bradycardia, hypotension, first } \\
\text { degree heart block, multifocal } \\
\text { PVC, VT }\end{array}$ & $\begin{array}{l}20 \%, \\
\text { Liposyn }\end{array}$ & $\begin{array}{l}250 \mathrm{~mL}(1.43 \\
\mathrm{g} / \mathrm{kg} / \mathrm{hr}) \times 2 \text { infusion, } \\
\text { each in } 30 \mathrm{~min}\end{array}$ & No & $\begin{array}{l}\text { Atropine } 1 \mathrm{mg} \text {, midazolam } 4 \\
\text { mg. ACLS protocol). } \\
\text { Intubation, oxygen, manual } \\
\text { ventilation }\end{array}$ & $\begin{array}{l}\text { Resolution of cardiac } \\
\text { symptoms }\end{array}$ & $\begin{array}{l}\text { Survival, } \\
\text { no sequelae }\end{array}$ \\
\hline Warren, 2008 (78) & \begin{tabular}{|l} 
Case \\
report
\end{tabular} & $\begin{array}{l}60 \mathrm{y} / \mathrm{M}, \\
83 \mathrm{~kg}\end{array}$ & $\begin{array}{l}\text { Mepivacaine } 450 \mathrm{mg} \text {, } \\
\text { Bupivacaine } 50 \mathrm{mg}\end{array}$ & $\begin{array}{l}1.40 \\
2.68\end{array}$ & Nerve block & $\begin{array}{l}\text { Unresponsiveness. } \\
\text { Cardiac arrest. } \\
\text { Labored respiration }\end{array}$ & $\begin{array}{l}20 \%, \\
\text { Liposyn III }\end{array}$ & $\left|\begin{array}{l}250 \mathrm{~mL}(1.2 \mathrm{~g} / \mathrm{kg} / \mathrm{hr}) \\
\text { infusion in } 30 \mathrm{~min}\end{array}\right|$ & 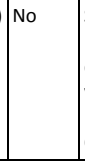 & $\begin{array}{l}\text { Sodium bicarbonate } 8.4 \% \\
100 \mathrm{~mL} \text {, atropine } 1 \mathrm{mg} \text {, } \\
\text { epinephrine } 1 \mathrm{mg} \times 3 \text {, } \\
\text { vasopressin } 40 \mathrm{U}, \\
\text { magnesium sulfate } 6 \mathrm{~g} \\
\text { CPR, defibrillation } \times 11\end{array}$ & $\begin{array}{l}\text { Longer intervals of } \\
\text { sustained cardiac } \\
\text { rythm during } \\
\text { defibrillation }\end{array}$ & $\begin{array}{l}\text { Survival, } \\
\text { no sequelae }\end{array}$ \\
\hline
\end{tabular}


AACT manuscript LA to edit for CTX

\begin{tabular}{|c|c|c|c|c|c|c|c|c|c|c|c|c|}
\hline Reference & $\begin{array}{l}\text { Study } \\
\text { type }\end{array}$ & $\begin{array}{l}\text { Age/sex, } \\
\text { weight }\end{array}$ & $\begin{array}{l}\text { Local } \\
\text { anesthetic and } \\
\text { dose }\end{array}$ & $\begin{array}{l}\log D \\
(129)\end{array}$ & $\begin{array}{l}\text { Route of } \\
\text { administration }\end{array}$ & Symptoms & ILE used & ILE dose\# & $\begin{array}{l}\text { ILE } \\
\text { only } \\
\text { used }\end{array}$ & $\begin{array}{l}\text { Other treatments } \\
\text { received, dose } \\
\text { included if } \\
\text { reported }\end{array}$ & ILE effect & Outcome \\
\hline Whiteman 2014 (79) & \begin{tabular}{|l|} 
Case \\
report
\end{tabular} & $\begin{array}{l}32 \mathrm{y} / \mathrm{F}, \\
62 \mathrm{~kg}\end{array}$ & Bupivacaine $870 \mathrm{mg}$ & 2.68 & Nerve block & $\begin{array}{l}\text { Confusion, agitation, } \\
\text { combative then seizures, } \\
\text { cardiac arrhythmia }\end{array}$ & \begin{tabular}{|l|}
$20 \%$ \\
Intralipid
\end{tabular} & $\begin{array}{l}1.5 \mathrm{~mL} / \mathrm{kg}(0.3 \mathrm{~g} / \mathrm{kg}) \\
\text { bolus then } 0.25 \\
\mathrm{~mL} / \mathrm{kg} / \mathrm{min}(3.0 \\
\mathrm{g} / \mathrm{kg} / \mathrm{hr}) \text { for } 60 \mathrm{~min}\end{array}$ & No & $\begin{array}{l}\text { Unspecified medical } \\
\text { therapy, cardiac } \\
\text { defibrillation } \times 2 \text {, surgery } \\
\text { with evacuation of } 60 \mathrm{~mL} \\
\text { fluid from the right rectus } \\
\text { sheath } \\
\text { CPR }\end{array}$ & $\begin{array}{l}\text { ROSC and normocardia } \\
\text { after } 45 \mathrm{~min} \text {. } \\
\text { Resolution of cardiac } \\
\text { arrythmia the following } \\
\text { day }\end{array}$ & $\begin{array}{l}\text { Survival, } \\
\text { no sequelae }\end{array}$ \\
\hline Whiteside, 2008 (80) & $\begin{array}{l}\text { Case } \\
\text { report }\end{array}$ & $\begin{array}{l}\text { Elderly/F, } \\
74 \mathrm{~kg}\end{array}$ & $\begin{array}{l}\text { Levobupivacaine } 21.65 \\
\text { mg }\end{array}$ & 2.68 & Nerve block & Seizure. & $\begin{array}{l}20 \% \\
\text { Intralipid }\end{array}$ & \begin{tabular}{|l|}
$100 \mathrm{~mL}(1.5 \mathrm{~mL} / \mathrm{kg}$ \\
$(0.30 \mathrm{~g} / \mathrm{kg}))$ bolus
\end{tabular} & Yes & $\begin{array}{l}\text { No pharmaceuticals } \\
\text { Oxygen, manual ventilation }\end{array}$ & $\begin{array}{l}\text { Unclear of effect is } \\
\text { related to ILE }\end{array}$ & $\begin{array}{l}\text { Survival, } \\
\text { no sequelae }\end{array}$ \\
\hline Widfeldt 2014 (81) & \begin{tabular}{|l} 
Case \\
report
\end{tabular} & $\begin{array}{l}62 \mathrm{y} / \mathrm{F}, \\
\text { weight NR }\end{array}$ & Ropivacaine $150 \mathrm{mg}$ & 4.21 & Nerve block & $\begin{array}{l}\text { Unconsciousness, nystagmus, } \\
\text { muscle twitching }\end{array}$ & \begin{tabular}{|l}
$20 \%$ \\
Intralipid
\end{tabular} & $\begin{array}{l}100 \mathrm{~mL}(1.5 \mathrm{~mL} / \mathrm{kg}) \\
\times 2 \text { bolus }-10 \mathrm{~min} \\
\text { interval, then } 50 \\
\mathrm{~mL} / \mathrm{hr} \text { for } 10 \mathrm{hrs} \\
\end{array}$ & No & \begin{tabular}{|l} 
Diazepam, few doses \\
(specific dose NR)
\end{tabular} & $\begin{array}{l}\text { Resolution of } \\
\text { neurologic symptoms }\end{array}$ & $\begin{array}{l}\text { Survival, } \\
\text { no sequelae }\end{array}$ \\
\hline Wong, 2010 (82) & \begin{tabular}{|l|} 
Case \\
report
\end{tabular} & $\begin{array}{l}6 \mathrm{y} / \mathrm{M}, \\
24 \mathrm{~kg}\end{array}$ & Bupivacaine, dose NR & 2.68 & Nerve block & $\begin{array}{l}\text { Sinus bradycardia }(60 / \mathrm{min}) \\
\text { that rapidly proceeded to a } \\
\text { wide complex ventricular } \\
\text { arrhythmia at } 40 / \text { min and } \\
\text { hypotension to } \mathrm{BP} 65 / 35 \\
\text { mmHg and tachycardia } \\
120 / \text { min }\end{array}$ & \begin{tabular}{|l|}
$20 \%$ \\
Intralipid
\end{tabular} & $\begin{array}{l}20 \mathrm{~mL}(0.17 \mathrm{~g} / \mathrm{kg}) \\
\text { bolus }\end{array}$ & No & $\begin{array}{l}\text { Crystalloid fluid boluses } 20 \\
\mathrm{~mL} / \mathrm{kg} \text {, atropine } 0.4 \mathrm{mg} \text {, } \\
\text { epinephrine } 0.2 \mathrm{mg} \text { then } \\
\text { continued } 0.1 \mathrm{mg} \text { boluses to } \\
\text { maintain a systolic pressure } \\
>60 \mathrm{mmHg} \text { then } 0.2 \\
\mathrm{\mu g} / \mathrm{kg} / \mathrm{min} \text { infusion. } \\
\text { Packed red celll }(30 \mathrm{~mL})+ \\
5 \% \text { albumin }(250 \mathrm{~mL}) \\
\text { CPR } \\
\end{array}$ & $\begin{array}{l}\text { Resolution of cardiac } \\
\text { symptoms }\end{array}$ & \begin{tabular}{|l|} 
Survival, \\
no sequelae for 3 days. \\
After 8 days, brain stem \\
death from cerebral \\
isshemia not related to ILE \\
treatment
\end{tabular} \\
\hline Zhurda, 2010 (83) & \begin{tabular}{|l|} 
Case \\
report
\end{tabular} & $\begin{array}{l}78 \mathrm{y} / \mathrm{M}, \\
62 \mathrm{~kg}\end{array}$ & Bupivacaine $100 \mathrm{mg}$ & 2.68 & Nerve block & $\begin{array}{l}\text { Perioral numbness, muscle } \\
\text { twitching, agitation, difficult } \\
\text { accommodation } \\
\text { HR 38/min which became wide } \\
\text { complex and hypotension to } \\
75 / 35 \mathrm{mmHH}\end{array}$ & \begin{tabular}{|l}
$20 \%$ \\
Intralipid
\end{tabular} & $\begin{array}{l}60 \mathrm{~mL}(0.19 \mathrm{~g} / \mathrm{kg}) \\
\text { bolus }\end{array}$ & No & $\begin{array}{l}\text { Midazolam } 3 \mathrm{mg} \text {, atropine } \\
\text { Oxygen }\end{array}$ & $\begin{array}{l}\text { Resolution of cardiac } \\
\text { symptoms }\end{array}$ & $\begin{array}{l}\text { Survival, } \\
\text { no sequelae }\end{array}$ \\
\hline Zimmer, 2007 (84) & \begin{tabular}{|l|} 
Case \\
report
\end{tabular} & $\begin{array}{l}84 \mathrm{y} / \mathrm{F}, \\
53 \mathrm{~kg}\end{array}$ & \begin{tabular}{|l|} 
Bupivacaine $43 \mathrm{mg}$ \\
\end{tabular} & 2.68 & Nerve block & $\begin{array}{l}\text { Agitation, confusion, restless. } \\
\text { Seizure. } \\
\text { Supraventicular tachycardia } \\
\text { (150/min) ventricular extra } \\
\text { systole, hypertension (170/85 } \\
\text { mmHg) } \\
\text { Heat sense in feet } \\
\end{array}$ & \begin{tabular}{|l}
$20 \%$ \\
Lipofundin
\end{tabular} & $\begin{array}{l}100 \mathrm{~mL}(0.38 \mathrm{~g} / \mathrm{kg}) \\
\text { bolus then } 0.5 \\
\mathrm{~mL} / \mathrm{kg} / \mathrm{hr}(0.1 \\
\mathrm{g} / \mathrm{kg} / \mathrm{hr})\end{array}$ & No & \begin{tabular}{|l|} 
Clonidin $150 \mathrm{mcg}$, \\
midazolam $5 \mathrm{mg}$, lidocain \\
$100 \mathrm{mg}$, propofol $1 \% 50 \mathrm{mg}$ \\
×2
\end{tabular} & $\begin{array}{l}\text { Resolution of cardiac } \\
\text { and neurologic } \\
\text { symptoms }\end{array}$ & $\begin{array}{l}\text { Survival, } \\
\text { no sequelae }\end{array}$ \\
\hline
\end{tabular}


AACT manuscript LA to edit for CTX

Table 2: Reported local anesthetics in the 16 volunteers from the randomized controlled study and the 83 patients from case reports and case series included in the systematic review.

\begin{tabular}{|l|l|l|l|}
\hline Local anesthetic & $\begin{array}{l}\text { Reported } \\
\text { cases, } \mathrm{n}\end{array}$ & Combination local anesthetics & $\begin{array}{l}\text { Reported } \\
\text { cases, } \mathrm{n}\end{array}$ \\
\hline Lidocaine** & 8 & Mepivacaine/Prilocaine & 1 \\
\hline Bupivacaine & 26 & Mepivacaine/Ropivacaine & 3 \\
\hline Mepivacaine & 4 & Lidocaine**/Ropivacaine & 5 \\
\hline Ropivacaine & $33^{*}$ & Lidocaine**/Levobupivacaine & 1 \\
\hline Levobupivacaine & $18^{*}$ & Lidocaine**/Prilocaine & 1 \\
\hline & & Bupivacaine/Lidocaine** & 9 \\
\hline & & Bupivacaine/Mepivacaine & 3 \\
\hline & & Bupivacaine/Ropivacaine & 2 \\
\hline & & Bupivacaine/Prilocaine & 1 \\
\hline
\end{tabular}

*The 16 volunteers received ropivacaine and levobupivacaine on each occasion, both are included in the table.

**Note: Lidocaine and lignocaine are synonyms : lidocaine is used in the table. 
AACT manuscript LA to edit for CTX

\section{Table 3. Summary of the 38 animal studies included in the systematic review on the effect of ILE}

* Drugs used for general anesthesia or euthanasia are not included.

ACLS: Advanced cardiac life support, AH: Atrial-His interval, ATP:Adenosine triphosphate, AUC: Area under the curve, BP: Blood pressure, CI: Cardiac index, CVP: Central venous pressure, CVT: CVT-4325, ECG: electrocardiogram, EPI: Epinephrine, HR: Heart rate, HV: His-ventricle interval, ILE: Intravenous lipid emulsion, IO: Intraosseous, IV: Intravenous, LA: Local anesthetic, LCT: Long chain triglyceride, LVdP/dtmax: maximal first derivative of left ventricular pressure, LVEDP: Left ventricular end-diastolic pressure, MAoP: Mean aortic pressure, MAP: Mean arterial pressure, MCT: Medium chain triglyceride, mPAP: Mean pulmonary artery pressure, NA: Not applicable, NR: Not reported, PCP: Pulmonary capillary pressure, pHm: Myocardial pH, Pm02: Myocardial tissue oxygen pressure, PVRI: Pulmonary vascular resistance index, RCS: Randomized controlled studies, ROSC: Return of spontaneous circulation, RPP: Rate pressure product, RR: Cardiac cycle length, std CPR: Standard cardio-pulmonary resuscitation, std resusc: Standard resuscitation, SVRI: Systemic vascular resistance index, VASO: Vasopressin \#: The bolus dose in $\mathrm{g} / \mathrm{kg}$ and infusion dose in $\mathrm{g} / \mathrm{kg} / \mathrm{hronly}$ be calculated if lipid concentration was reported.

\begin{tabular}{|c|c|c|c|c|c|c|c|c|c|c|c|c|c|}
\hline $\begin{array}{l}\text { Reference } \\
\text { (Species) }\end{array}$ & Model & $\begin{array}{l}\text { Local } \\
\text { anesthetic } \\
\text { (dose) }\end{array}$ & $\left.\mid \begin{array}{l}\text { Log } \\
D \\
(129)\end{array}\right)$ & Symptoms & $\begin{array}{l}\text { ILE } \\
\text { used }\end{array}$ & $\begin{array}{l}\text { ILE } \\
\text { bolus \# }\end{array}$ & $\begin{array}{l}\text { ILE } \\
\text { infusion } \\
\#\end{array}$ & Study arms & $\begin{array}{l}\text { Timing of } \\
\text { rescue, } \\
\text { time from } \\
\text { LA } \\
\text { termination }\end{array}$ & $\begin{array}{l}\text { Other } \\
\text { treatments } \\
\text { received * }\end{array}$ & $\begin{array}{l}\text { Parameter } \\
\text { measured }\end{array}$ & Outcome & $\begin{array}{l}\text { Support } \\
\text { therapeutic } \\
\text { effect of ILE } \\
\text { alone }\end{array}$ \\
\hline \multicolumn{14}{|l|}{ RCS } \\
\hline $\begin{array}{l}\begin{array}{l}\text { Bonfim, } 2012 \text { (85) } \\
\text { (Pig) }\end{array} \\
\text { (ig) }\end{array}$ & \begin{tabular}{|l|} 
RCS; \\
compared LCT \\
and MCT/LCT
\end{tabular} & \begin{tabular}{|l} 
Ropivacaine \\
$(7 \mathrm{mg} / \mathrm{kg}$ in 30 \\
$\mathrm{sec})$
\end{tabular} & 4.21 & $\begin{array}{l}\text { Decrease in } \\
\text { mean arterial } \\
\text { pressure }\end{array}$ & \begin{tabular}{|l|}
$20 \%$, \\
Lipovenos \\
MCT and \\
Lipovenos
\end{tabular} & \begin{tabular}{|l|}
$4 \mathrm{~mL} / \mathrm{kg}$ \\
$(0.8 \mathrm{~g} / \mathrm{kg})$
\end{tabular} & No & $\begin{array}{l}\text { MCT/LCT ILE } \\
\text { vs } \\
\text { LCT ILE } \\
\text { vs } \\
\text { Saline }\end{array}$ & \begin{tabular}{|l|}
$\begin{array}{l}\text { Other } \\
\text { treatment and } \\
\text { study treatment } \\
\text { at } 1 \text { min. }\end{array} \mid$ | \\
\end{tabular} & $\begin{array}{l}\text { Vasopressors: } \\
0.8 \mathrm{~g} / \mathrm{kg}\end{array}$ & $\begin{array}{l}\text { At } 30 \text { min increase MAP } \\
\text { (LCT = MCT/LCT), CI (only } \\
\text { MCT/LCT), SVRI (LCT = } \\
\text { MCT/LCT), PVRI (only } \\
\text { MCT/LCT); no effect HR, } \\
\text { CVP, mPAP, PCP }\end{array}$ & Survival: All & $\begin{array}{l}\text { Yes; For both LCT and } \\
\text { MCT/LCT }\end{array}$ \\
\hline \begin{tabular}{|l|} 
Buckenmaier, 2012 \\
$(17)$ \\
(Pig)
\end{tabular} & \begin{tabular}{|l|} 
RCS; \\
post mortem \\
distribution study
\end{tabular} & \begin{tabular}{|l|} 
Ropivacaine \\
$(1.5 \mathrm{mg} / \mathrm{kg} / \mathrm{min})$
\end{tabular} & 4.21 & Asystole & \begin{tabular}{|l|}
$20 \%$, \\
Intralipid
\end{tabular} & \begin{tabular}{|l|}
$1 \mathrm{~mL} / \mathrm{kg}$ \\
$(0.2 \mathrm{~g} / \mathrm{kg})$
\end{tabular} & No & \begin{tabular}{|l|} 
ILE \\
vs \\
No ILE
\end{tabular} & \begin{tabular}{|l|l}
$\begin{array}{l}\text { LA and study } \\
\text { treatment were } \\
\text { dosed } \\
\text { simultaneously }\end{array}$ & r \\
\end{tabular} & $\begin{array}{l}\text { Saline 1-2 } \\
\mathrm{mL} / \mathrm{kg} / \mathrm{hr}\end{array}$ & \begin{tabular}{|l|} 
Asystole; \\
Earlier onset of death \\
(asystole) in ILE compared \\
to non-ILE \\
\end{tabular} & $\begin{array}{l}\text { Survival: ILE 0/6, } \\
\text { No ILE 0/6 }\end{array}$ & No; Post-mortem study \\
\hline $\begin{array}{l}\begin{array}{l}\text { Bushey, } 2011 \text { (86) } \\
\text { (Pig) }\end{array} \\
\text { (Pig) }\end{array}$ & \begin{tabular}{|l|} 
RCS; \\
resuscitation \\
model
\end{tabular} & $\begin{array}{l}\text { Bupivacaine } \\
(5 \mathrm{mg} / \mathrm{kg})\end{array}$ & 2.68 & $\begin{array}{l}\text { Cardiovascular } \\
\text { collapse }\end{array}$ & \begin{tabular}{|l|}
$20 \%$ \\
Intralipid
\end{tabular} & $\begin{array}{l}4 \mathrm{~mL} / \mathrm{kg} \\
(0.8 \mathrm{~g} / \mathrm{kg})\end{array}$ & \begin{tabular}{|l|}
0.5 \\
$\mathrm{~mL} / \mathrm{kg} / \mathrm{min}$ \\
$66 \mathrm{~g} / \mathrm{kg} / \mathrm{hr})$ \\
for $10 \mathrm{~min}$
\end{tabular} & \begin{tabular}{|l|} 
ILE \\
vs \\
Saline
\end{tabular} & \begin{tabular}{|l|l} 
Other \\
treatments at 4 \\
min., then \\
study treatment
\end{tabular} & \begin{tabular}{|l|} 
ACLS \\
resuscitation \\
and closed \\
chest \\
compression \\
\end{tabular} & \begin{tabular}{|l|} 
ROSC (unsupported systolic \\
BP of $60 \mathrm{mmHg}$ or greater \\
for $10 \mathrm{~min}$ )
\end{tabular} & $\begin{array}{l}\text { Survival: ILE 6/12, } \\
\text { Saline 4/12 }\end{array}$ & $\begin{array}{l}\text { No; suggest that the } \\
\text { addition of ILE to ACLS } \\
\text { intervention does not } \\
\text { improve survival }\end{array}$ \\
\hline \begin{tabular}{|l|} 
Candela, $2010(87)$ \\
(Pig)
\end{tabular} & $\begin{array}{l}\text { RCS; } \\
\text { resuscitation } \\
\text { model }\end{array}$ & $\begin{array}{l}\text { Bupivacaine } \\
(4 \mathrm{mg} / \mathrm{kg})\end{array}$ & 2.68 & \begin{tabular}{|l|} 
Lengthening of \\
HV, QRS, AH and \\
Pa intervals, no \\
alteration in RR \\
and JT intervals. \\
Haemodynamics: \\
decrease in \\
LVdP/dtmax, \\
increase in \\
LVEDP, no \\
change in MAoP
\end{tabular} & $\left|\begin{array}{l}20 \% \\
\text { Medialip } \\
\text { and Ivelip }\end{array}\right|$ & $\begin{array}{l}1.5 \mathrm{~mL} / \mathrm{kg} \\
(0.3 \mathrm{~g} / \mathrm{kg})\end{array}$ & $\begin{array}{l}0.25 \\
\mathrm{~m} / / \mathrm{kg} / \mathrm{min} \\
(3 \mathrm{~g} / \mathrm{kg} / \mathrm{hr})\end{array}$ & $\mid \begin{array}{l}\text { (MCT/LCT and LCT) } \\
\text { ILE+Saline } \\
\text { vs } \\
\text { Saline }\end{array}$ & $\begin{array}{l}\text { Study } \\
\text { treatment at } 30 \\
\text { sec. }\end{array} \mid$ & No & $\begin{array}{l}\text { Hemodynamics : LCT and } \\
\text { MCT/LCT - MAoP and } \\
\text { LVdP/dTmax were } \\
\text { increased by ILE therapy } \\
\text { when comparing AUC; } \\
\text { QRS width: LCT and } \\
\text { MCT/LCT - Effects on QRS } \\
\text { duration was reversed }\end{array}$ & $\begin{array}{l}\text { Survival: LCT 7/7, } \\
\text { MCT/LCT 8/8, } \\
\text { Saline 9/9 }\end{array}$ & Yes \\
\hline
\end{tabular}


AACT manuscript LA to edit for CTX

\begin{tabular}{|c|c|c|c|c|c|c|c|c|c|c|c|c|c|}
\hline $\begin{array}{l}\text { Reference } \\
\text { (Species) }\end{array}$ & Model & \begin{tabular}{|l} 
Local \\
anesthetic \\
(dose)
\end{tabular} & \begin{tabular}{|l|} 
Log \\
$D$ \\
$(129)$
\end{tabular} & Symptoms & $\begin{array}{l}\text { ILE } \\
\text { used }\end{array}$ & \begin{tabular}{|l|} 
ILE \\
bolus \#
\end{tabular} & \begin{tabular}{|l} 
ILE \\
infusion \\
$\#$
\end{tabular} & Study arms & \begin{tabular}{|l|} 
Timing of \\
rescue, \\
time from \\
LA \\
termination
\end{tabular} & \begin{tabular}{|l|} 
Other \\
treatments \\
received *
\end{tabular} & $\begin{array}{l}\text { Parameter } \\
\text { measured }\end{array}$ & Outcome & \begin{tabular}{|l} 
Support \\
therapeutic \\
effect of ILE \\
alone
\end{tabular} \\
\hline $\begin{array}{l}\text { De Queiroz, } 2012 \\
\text { (88) ฉ } 2 \\
\text { (Pig) }\end{array}$ & \begin{tabular}{|l|} 
RCS; \\
resuscitation \\
model
\end{tabular} & $\begin{array}{l}\text { Levobupivacaine } \\
\text { (500 mg/hr until } \\
\text { symptoms) }\end{array}$ & 2.68 & $\begin{array}{l}\text { MAP decrease by } \\
50 \% \text { for } 15 \mathrm{sec}\end{array}$ & \begin{tabular}{|l|}
$20 \%$, \\
brand NR
\end{tabular} & \begin{tabular}{|l|}
$4 \mathrm{~mL} / \mathrm{kg}$ \\
$(0.8 \mathrm{~g} / \mathrm{kg})$ \\
\end{tabular} & $\begin{array}{l}0.25 \\
\mathrm{~mL} / \mathrm{kg} / \mathrm{min} \\
(3 \mathrm{~g} / \mathrm{kg} / \mathrm{hr})\end{array}$ & \begin{tabular}{|l} 
ILE \\
vs \\
ILE+EPI \\
vs \\
EPI \\
vs \\
Control (no \\
additional drugs)
\end{tabular} & NR & $\begin{array}{l}\text { EPI } 10 \mathrm{mg} / \mathrm{kg} \\
\text { every } 3 \mathrm{~min}\end{array}$ & \begin{tabular}{|l|} 
Hemodynamics: \\
Cardiovascular collapse \\
defined by a decrease in \\
MAP by 50\%; \\
EPI alone or in combination \\
with ILE was associated \\
with rhythmic or \\
conduction cardiac \\
disturbances
\end{tabular} & $\begin{array}{l}\text { Survival: ILE 7/9, } \\
\text { ILE+EPI 10/10, } \\
\text { EPI 6/7, } \\
\text { Control 1/7, }\end{array}$ & Yes \\
\hline $\begin{array}{l}\text { De Queiroz, } 2014 \\
\text { (89) } \\
\text { (Pig) }\end{array}$ & \begin{tabular}{|l|} 
RCS; \\
resuscitation \\
model
\end{tabular} & \begin{tabular}{|l|} 
Levobupivacaine \\
$(8.3 \mathrm{mg} / \mathrm{min})$
\end{tabular} & 2.68 & $\begin{array}{l}\text { MAP decreased } \\
\text { to } 50 \% \text { of its } \\
\text { baseline value }\end{array}$ & \begin{tabular}{|l|}
$20 \%$ \\
Intralipid
\end{tabular} & \begin{tabular}{|l|}
$4 \mathrm{~mL} / \mathrm{kg}$ \\
$(0.8 \mathrm{~g} / \mathrm{kg})$ \\
\end{tabular} & $\begin{array}{l}0.25 \\
\mathrm{~mL} / \mathrm{kg} / \mathrm{min} \\
(3 \mathrm{~g} / \mathrm{kg} / \mathrm{hr})\end{array}$ & \begin{tabular}{|l} 
ILE \\
vs \\
EPI \\
vs \\
lLE+EPI \\
vs \\
Saline \\
\end{tabular} & \begin{tabular}{|l|l} 
Std CPR & $S$ \\
immediately, & \\
then study & \\
treatment & a \\
\end{tabular} & \begin{tabular}{|l|} 
Std CPR (chest \\
compressions \\
and manual \\
ventilation)
\end{tabular} & \begin{tabular}{|l|} 
Time to ROSC in survivors: \\
ILE 460 sec, EPI 296 sec, \\
ILE+EPI 304 sec, Saline 720 \\
sec \\
ECG abnormalities \\
(arrhythmia; conduction) \\
number, after ROSC in \\
survivors: \\
ILE (0;0), EPI (11;3), ILE+EPI \\
$(10 ; 7)$
\end{tabular} & \begin{tabular}{|l|} 
Survival: ILE $7 / 9$, \\
EPI 6/7, \\
ILE+EPI 10/10, \\
Saline 1/7
\end{tabular} & \begin{tabular}{|l|} 
Yes; ILE, EPI, and \\
ILEEEPI provided \\
similar ROSC. ECG \\
abnormalities from EPI \\
or ILE+EPI increased \\
compared to ILE
\end{tabular} \\
\hline $\begin{array}{l}\text { Di Gregorio, } 2008 \\
\text { (91) } a \\
\text { (Rats) }\end{array}$ & \begin{tabular}{|l|} 
RCS; \\
resuscitation \\
model
\end{tabular} & $\begin{array}{l}\text { Bupivacaine } \\
(20 \mathrm{mg} / \mathrm{kg})\end{array}$ & 2.68 & Cardiac arrest & \begin{tabular}{|l|}
$30 \%$, \\
Soy bean \\
oil \\
emulsion
\end{tabular} & \begin{tabular}{|l|}
$\mathrm{mL} / \mathrm{kg}$ \\
$(1.5 \mathrm{~g} / \mathrm{kg})$ \\
\end{tabular} & $\begin{array}{l}0.5 \\
\mathrm{~mL} / \mathrm{kg} / \mathrm{min} \\
(9 \mathrm{~g} / \mathrm{kg} / \mathrm{hr})\end{array}$ & \begin{tabular}{|l} 
ILE \\
vs \\
EPI \\
vs \\
Saline
\end{tabular} & NR & $\begin{array}{l}\text { cardiac } \\
\text { resuscitation }\end{array}$ & \begin{tabular}{|l|} 
QRS width: Bupivacaine- \\
induced QRS prolongation \\
reverted to normal in both \\
ILE and EPI groups but \\
persisted in Saline group at \\
10 min
\end{tabular} & $\begin{array}{l}\text { ROSC: ILE 5/5, } \\
\text { EPI 4/5, } \\
\text { Saline 2/5 }\end{array}$ & Yes \\
\hline $\begin{array}{l}\text { Di Gregorio, } 2009 \\
\text { (92) } \\
\text { (Rats) }\end{array}$ & \begin{tabular}{|l|} 
RCS; \\
resuscitation \\
model
\end{tabular} & $\begin{array}{l}\text { Bupivacaine } \\
(20 \mathrm{mg} / \mathrm{kg})\end{array}$ & 2.68 & Cardiac arrest & \begin{tabular}{|l|}
$30 \%$, \\
Intralipid
\end{tabular} & \begin{tabular}{|l|}
$5 \mathrm{~mL} / \mathrm{kg}$ \\
$(1.5 \mathrm{~g} / \mathrm{kg})$, \\
repeated \\
at 2.5 and \\
$5 \mathrm{~min}$ \\
\end{tabular} & $\begin{array}{l}1 \\
\mathrm{~mL} / \mathrm{kg} / \mathrm{min} \\
(18 \mathrm{~g} / \mathrm{kg} / \mathrm{hr})\end{array}$ & \begin{tabular}{|l} 
ILE \\
vs \\
VASO \\
vs \\
VASO+EPI
\end{tabular} & \begin{tabular}{l|l} 
Other \\
treatment and \\
study treatment \\
immediately \\
after LA
\end{tabular} & \begin{tabular}{|l|} 
VASO $0.4 \mathrm{U} / \mathrm{kg}$, \\
EPI $30 \mathrm{~g} / \mathrm{kg}$, \\
chest \\
compression, \\
mechanical \\
ventilation
\end{tabular} & \begin{tabular}{|l|} 
Hemodynamics: Rate \\
pressure product higher in \\
ILE vs VASO and VASO+EPI; \\
QRS width: ILE group \\
returned to baseline
\end{tabular} & \begin{tabular}{|l|} 
Survival: NR \\
\end{tabular} & \begin{tabular}{|l|} 
Yes; ILE resuscitation \\
was superior to \\
vasopressors (VASO \\
and VASO+EPI) in \\
treating bupivacaine- \\
induced asystole. \\
Adverse events higher \\
in vasopressor group
\end{tabular} \\
\hline
\end{tabular}


AACT manuscript LA to edit for CTX

\begin{tabular}{|c|c|c|c|c|c|c|c|c|c|c|c|c|c|}
\hline $\begin{array}{l}\text { Reference } \\
\text { (Species) }\end{array}$ & Model & $\begin{array}{l}\text { Local } \\
\text { anesthetic } \\
\text { (dose) }\end{array}$ & $\left.\mid \begin{array}{l}\text { Log } \\
D \\
(129)\end{array}\right)$ & Symptoms & $\begin{array}{l}\text { ILE } \\
\text { used }\end{array}$ & \begin{tabular}{|l|} 
ILE \\
bolus \#
\end{tabular} & \begin{tabular}{|l} 
ILE \\
infusion \\
$\#$
\end{tabular} & Study arms & $\begin{array}{l}\text { Timing of } \\
\text { rescue, } \\
\text { time from } \\
\text { LA } \\
\text { termination }\end{array}$ & $\begin{array}{l}\text { Other } \\
\text { treatments } \\
\text { received * }\end{array}$ & $\begin{array}{l}\text { Parameter } \\
\text { measured }\end{array}$ & Outcome & $\begin{array}{l}\text { Support } \\
\text { therapeutic } \\
\text { effect of ILE } \\
\text { alone }\end{array}$ \\
\hline $\begin{array}{l}\text { Fettiplace, } 2014 \\
\text { (93) } \\
\text { (Rats) }\end{array}$ & $\begin{array}{l}\text { RCS; } \\
\text { resuscitation } \\
\text { model }\end{array}$ & \begin{tabular}{|l} 
Bupivacaine \\
$(10 \mathrm{mg} / \mathrm{kg})$
\end{tabular} & 2.68 & \begin{tabular}{|l|} 
Transient \\
cardiovascular \\
toxicity
\end{tabular} & \begin{tabular}{|l|}
$20 \%$ and \\
$30 \%$ \\
Intralipid \\
\end{tabular} & $\begin{array}{l}4 \mathrm{~mL} / \mathrm{kg} \\
(0.8 \mathrm{~g} / \mathrm{kg} \\
\text { and } \\
1.2 \mathrm{~g} / \mathrm{kg})\end{array}$ & No & \begin{tabular}{|l|}
$30 \%$ ILE \\
vs \\
$20 \%$ ILE \\
vs \\
$0.9 \%$ Saline \\
vs \\
Control (no \\
treatment)
\end{tabular} & \begin{tabular}{|l|} 
Study \\
treatment after \\
$10 \mathrm{sec}$
\end{tabular} & \begin{tabular}{|l|} 
Mechanical \\
ventilation
\end{tabular} & \begin{tabular}{|l|} 
Time to $50 \%$ recovery of \\
cardiovascular parameters \\
rate-pressure product \\
(RPP), MAP, Carotid flow \\
(flow), HR; All animals \\
returned to $50 \%$ RPP. Order \\
of mean recovery times: \\
ILE30<ILE20<Saline<Null. \\
HR recovered faster than \\
other measures \\
parameters. \\
\end{tabular} & \begin{tabular}{|l|} 
Survival: ILE30 7/7, \\
ILE20 7/7, \\
Saline 7/7, \\
Control 7/7 \\
\end{tabular} & Not studied \\
\hline $\begin{array}{l}\text { Fettiplace, } 2014 \\
\text { (94) } \\
\text { (Rats) }\end{array}$ & \begin{tabular}{|l|} 
RCS; \\
resuscitation \\
model
\end{tabular} & $\begin{array}{l}\text { Bupivacaine } \\
(10 \mathrm{mg} / \mathrm{kg} \text { in } 10 \\
\mathrm{sec})\end{array}$ & 2.68 & Asystole & \begin{tabular}{|l|}
$30 \%$ \\
Intralipid
\end{tabular} & \begin{tabular}{|l|}
1010 \\
$\mathrm{~mL} / \mathrm{kg}(3.0$ \\
$\mathrm{g} / \mathrm{kg}$ ) in \\
$180 \mathrm{sec} ; \mathrm{IV}$ \\
$10 \mathrm{~mL} / \mathrm{kg}$ \\
$(3.0 \mathrm{~g} / \mathrm{kg})$ \\
in $90 \mathrm{sec}$. \\
\end{tabular} & \begin{tabular}{|l}
0.5 \\
$\mathrm{~mL} / \mathrm{kg} / \mathrm{min}$ \\
$(9 \mathrm{~g} / \mathrm{kg} / \mathrm{hr})$
\end{tabular} & \begin{tabular}{|l|} 
ILE (IO) \\
vs \\
ILE(IV) \\
vs \\
Saline (IO)
\end{tabular} & \begin{tabular}{|l|} 
Study \\
treatment at 10 \\
sec.
\end{tabular} & No & $\begin{array}{l}\text { Hemodynamics: ECG, aortic } \\
\text { pressure, carotid blood } \\
\text { flow; Return of } 50 \% \text { flow; } \\
\text { Comparable recovery of } \\
\text { hemodynamic variables in } \\
\text { ILE (IO) and ILE (IV). Faster } \\
\text { recovery in ILE (IO) and ILE } \\
\text { (IV) compared to Saline and } \\
\text { no treatment }\end{array}$ & Survival: All & Yes \\
\hline $\begin{array}{l}\text { Hicks, } 2009 \text { (96) } \\
\text { (Pig) }\end{array}$ & $\begin{array}{l}\text { RCS; } \\
\text { resuscitation } \\
\text { model }\end{array}$ & \begin{tabular}{|l|} 
Bupivacaine \\
$(10 \mathrm{mg} / \mathrm{kg}$ in 10 \\
$\mathrm{sec})$
\end{tabular} & 2.68 & \begin{tabular}{|l|} 
Cardiac arrest, \\
then wide QRS \\
complex, \\
premature \\
ventricular \\
contractions, and \\
premature atrial \\
contractions \\
\end{tabular} & \begin{tabular}{|l|}
$20 \%$ \\
Intralipid
\end{tabular} & \begin{tabular}{|l|}
$4 \mathrm{~mL} / \mathrm{kg}$ \\
$(0.8 \mathrm{~g} / \mathrm{kg})$
\end{tabular} & $\begin{array}{l}0.5 \\
\mathrm{~mL} / \mathrm{kg} / \mathrm{min} \\
(6 \mathrm{~g} / \mathrm{kg} / \mathrm{hr})\end{array}$ & \begin{tabular}{|l} 
ILE \\
vs \\
Saline
\end{tabular} & \begin{tabular}{|l|} 
Other \\
treatments \\
immediately \\
after LA for 5 \\
min., then \\
study treatment
\end{tabular} & \begin{tabular}{|l|} 
EPI $(100 \mathrm{~g} / \mathrm{kg})$ \\
and VASO $(1.5$ \\
$\mathrm{U} / \mathrm{kg})$ \\
cardiac \\
resuscitation
\end{tabular} & \begin{tabular}{|l|} 
Hemodynamics: MAP: 82.9 \\
$+/-12.2$ (base line), 83.9 \\
$+/-10.4(15 \mathrm{~min}), 83.6+/-$ \\
$8.9(30 \mathrm{~min}), 80.2+/-13.7$ \\
$(45 \mathrm{~min}), 69.5+/-7.8(60$ \\
$\mathrm{min})$
\end{tabular} & $\begin{array}{l}\text { Survival: ILE 3/10, } \\
\text { Saline 4/9 }\end{array}$ & \begin{tabular}{|l} 
No; Adding ILE \\
resuscitation to EPI and \\
VASO did not improve \\
outcomes
\end{tabular} \\
\hline
\end{tabular}


AACT manuscript LA to edit for CTX

\begin{tabular}{|c|c|c|c|c|c|c|c|c|c|c|c|c|c|}
\hline $\begin{array}{l}\text { Reference } \\
\text { (Species) }\end{array}$ & Model & \begin{tabular}{|l} 
Local \\
anesthetic \\
(dose)
\end{tabular} & \begin{tabular}{|l} 
Log \\
$D$ \\
$(129)$
\end{tabular} & Symptoms & $\begin{array}{l}\text { ILE } \\
\text { used }\end{array}$ & \begin{tabular}{|l|} 
ILE \\
bolus \#
\end{tabular} & $\begin{array}{l}\text { ILE } \\
\text { infusion } \\
\#\end{array}$ & Study arms & \begin{tabular}{|l|} 
Timing of \\
rescue, \\
time from \\
LA \\
termination
\end{tabular} & \begin{tabular}{|l|} 
Other \\
treatments \\
received *
\end{tabular} & $\begin{array}{l}\text { Parameter } \\
\text { measured }\end{array}$ & Outcome & \begin{tabular}{|l} 
Support \\
therapeutic \\
effect of ILE \\
alone
\end{tabular} \\
\hline $\begin{array}{l}\text { Hiller, } 2009 \text { (97) } \\
\text { (Rats) }\end{array}$ & \begin{tabular}{|l|} 
RCS; \\
resuscitation \\
model
\end{tabular} & $\begin{array}{l}\text { Bupivacaine } \\
(20 \mathrm{mg} / \mathrm{kg})\end{array}$ & 2.68 & Asystole & \begin{tabular}{|l|}
$30 \%$ \\
Intralipid
\end{tabular} & \begin{tabular}{|l|}
$5 \mathrm{~mL} / \mathrm{kg}$ \\
$(1.5 \mathrm{~g} / \mathrm{kg})$ \\
$\mathrm{x} 2$
\end{tabular} & \begin{tabular}{|l|}
$1 \mathrm{~mL} / \mathrm{kg}(9$ \\
$\mathrm{g} / \mathrm{kg} / \mathrm{hr})$ for \\
$2 \mathrm{~min}$
\end{tabular} & \begin{tabular}{|l} 
ILE \\
vs \\
ILE+EPI \\
vs \\
Saline
\end{tabular} & \begin{tabular}{|l|} 
Other \\
treatment \\
immediately \\
after LA, study \\
treatment at 3 \\
min.
\end{tabular} & \begin{tabular}{|l|} 
Saline or ILE or \\
ILE+EPI 1, 2.5, \\
10 or 25 \\
$\mathrm{mcg} / \mathrm{kg}$.
\end{tabular} & \begin{tabular}{|l|} 
Hemodynamics: Manual \\
chest compressions to \\
achieve a rate-pressure \\
product (=systolic pressure \\
* HR) of at least $50 \%$ of \\
baseline; \\
EPI (up to $2.5 \mathrm{mcg} / \mathrm{kg}$ ) \\
improved initial ROSC but \\
few animals sustained by \\
15 min. ILE alone resulted \\
in slower but more \\
sustained recovery.
\end{tabular} & Survival: All & \begin{tabular}{|l|} 
No; ILE alone \\
compared to ILE+EPI \\
(1, 2.5, $10,25 \mathrm{mg} / \mathrm{kg})$. \\
ILE alone resulted in \\
ROSC, ILE+EPI doses \\
below 10 had faster \\
and more sustained \\
ROSC
\end{tabular} \\
\hline $\begin{array}{l}\text { Karci, } 2009(98) \text { म } \\
\text { (Rats) }\end{array}$ & \begin{tabular}{|l|} 
RCS; \\
resuscitation \\
model
\end{tabular} & \begin{tabular}{|l|}
$\begin{array}{l}\text { Levobupivacaine } \\
(3 \mathrm{mg} / \mathrm{kg} / \mathrm{min})\end{array}$ \\
\end{tabular} & 2.68 & \begin{tabular}{|l|} 
Decrease of 50\% \\
in mean BP. \\
Asystole
\end{tabular} & $\begin{array}{l}20 \%, \\
\text { brand NR }\end{array}$ & No & \begin{tabular}{|l}
$1.5 \mathrm{~mL} / \mathrm{kg}$ \\
$(0.6$ \\
$\mathrm{g} / \mathrm{kg} / \mathrm{hr}$ ) for \\
$30 \mathrm{~min}$ (one \\
group) time \\
NR for other \\
groups
\end{tabular} & \begin{tabular}{|l|} 
Post-treatment ILE \\
or Simultaneous- \\
treatment ILE \\
vs \\
no ILE \\
vs \\
No LA+ILE
\end{tabular} & \begin{tabular}{|l|} 
Other \\
treatment and \\
study treatment \\
immediately \\
after LA
\end{tabular} & \begin{tabular}{|l|} 
Standard \\
resuscitation \\
(unspecified)
\end{tabular} & \begin{tabular}{|l|} 
Hemodynamics: \\
No hemodynamic changes \\
were observed in rats \\
receiving only ILE emulsion; \\
Time to development of \\
asystole was longer \\
compared to other \\
levobupivacaine dosed \\
groups.
\end{tabular} & \begin{tabular}{|l|} 
Survival: ILE at 50\% \\
MAP+std resusc 4 \\
/7, \\
ILE+std resusc 1/7, \\
std resusc 0/7, \\
No LA+LLE $7 / 7$
\end{tabular} & \begin{tabular}{|l} 
Yes; Simultaneous \\
treatment and post \\
treatment parts \\
suggest that \\
administration of ILE \\
may prevent cardiac \\
arrest and ILE infusion \\
along with standard \\
resuscitation in cardiac \\
arrest may improve \\
survival
\end{tabular} \\
\hline \begin{tabular}{|l|}
$\begin{array}{l}\text { Karcioglu, } \\
\text { (Rabbit) }\end{array}$ \\
\end{tabular} & $\begin{array}{l}\text { RCS; } \\
\text { resuscitation } \\
\text { model }\end{array}$ & \begin{tabular}{|l|}
$\begin{array}{l}\text { Levobupivacaine } \\
(10 \mathrm{mg} / \mathrm{kg})\end{array}$ \\
\end{tabular} & 2.68 & Asystole & \begin{tabular}{|l|}
$20 \%$ \\
Intralipid
\end{tabular} & \begin{tabular}{|l|}
$.5 \mathrm{~mL} / \mathrm{kg}$ \\
$(0.3 \mathrm{~g} / \mathrm{kg})$, \\
additional \\
boluses, 5 \\
min \\
interval if \\
absebse of \\
ROSC
\end{tabular} & No & \begin{tabular}{|l|} 
ILE \\
vs \\
Saline
\end{tabular} & \begin{tabular}{|l|}
30 sec non- \\
intervention \\
period, then \\
study treatment
\end{tabular} & \begin{tabular}{|l|} 
Mechanical \\
ventilation, \\
manual chest \\
compression, \\
EPI $100 \mathrm{mcg} / \mathrm{kg}$ \\
every $5 \mathrm{~min}$
\end{tabular} & $\begin{array}{l}\text { ROSC (MAP }>50 \mathrm{mmHg} \text { and } \\
\text { HR>120 bpm): ILE>Saline. }\end{array}$ & $\begin{array}{l}\text { Survival : ILE 3/7, } \\
\text { Saline } 1 / 7\end{array}$ & $\begin{array}{l}\begin{array}{l}\text { Not studied; ILE+EPI } \\
\text { superior to EPI alone. }\end{array} \\
\end{array}$ \\
\hline $\begin{array}{l}\text { Li, } 2011 \text { (100) } \\
\text { (Rats) }\end{array}$ & \begin{tabular}{|l|} 
RCS; \\
resuscitation \\
model
\end{tabular} & $\begin{array}{l}\text { Bupivacaine } \\
(20 \mathrm{mg} / \mathrm{kg})\end{array}$ & 2.68 & Cardiac arrest & \begin{tabular}{|l}
$20 \%$, \\
Lipovenos \\
MCT and \\
Intralipid
\end{tabular} & \begin{tabular}{|l|}
$\mathrm{mL} / \mathrm{kg}(1$ \\
$\mathrm{g} / \mathrm{kg})$
\end{tabular} & $\begin{array}{l}1 \\
\mathrm{~mL} / \mathrm{kg} / \mathrm{min} \\
(12 \mathrm{~g} / \mathrm{kg} / \mathrm{hr}) \\
\text { for } 3 \mathrm{~min}\end{array}$ & \begin{tabular}{|l} 
MCT/LCT ILE \\
vs \\
LCT ILE
\end{tabular} & \begin{tabular}{|l|} 
Other \\
treatment and \\
study treatment \\
immediately \\
after LA \\
LA
\end{tabular} & $\begin{array}{l}\text { EPI } 40 \mathrm{mcg} / \mathrm{kg} \\
\text { (LCT), } 50 \\
\mathrm{mcg} / \mathrm{Kg} \\
\text { (MCT/LCT), } \\
\text { chest } \\
\text { compression, } \\
\text { mechanical } \\
\text { ventilation }\end{array}$ & $\begin{array}{l}\text { Hemodynamics: RPP more } \\
\text { than } 20 \% \text { of baseline value } \\
\text { for } 1 \mathrm{~min}=\mathrm{ROSC}\end{array}$ & \begin{tabular}{|l|} 
Mortality after \\
resusc lower in LCT \\
$(2 / 30)$ vs MCT/LCT \\
$(8 / 30)$
\end{tabular} & \begin{tabular}{|l|} 
Not studied; Supports \\
LCT over a MCT/LCT. \\
Did not have a control \\
without ILE \\
resuscitation
\end{tabular} \\
\hline
\end{tabular}


AACT manuscript LA to edit for CTX

\begin{tabular}{|c|c|c|c|c|c|c|c|c|c|c|c|c|c|}
\hline $\begin{array}{l}\text { Reference } \\
\text { (Species) }\end{array}$ & Model & \begin{tabular}{|l} 
Local \\
anesthetic \\
(dose)
\end{tabular} & $\left|\begin{array}{l}\log \\
D \\
(129)\end{array}\right|$ & Symptoms & $\begin{array}{l}\text { ILE } \\
\text { used }\end{array}$ & $\begin{array}{l}\text { ILE } \\
\text { bolus \# }\end{array}$ & \begin{tabular}{|l} 
ILE \\
infusion \\
$\#$
\end{tabular} & Study arms & \begin{tabular}{|l|} 
Timing of \\
rescue, \\
time from \\
LA \\
termination
\end{tabular} & $\begin{array}{l}\text { Other } \\
\text { treatments } \\
\text { received * }\end{array}$ & $\begin{array}{l}\text { Parameter } \\
\text { measured }\end{array}$ & Outcome & $\begin{array}{l}\text { Support } \\
\text { therapeutic } \\
\text { effect of ILE } \\
\text { alone }\end{array}$ \\
\hline \begin{tabular}{|l|} 
Litonius, 2012 (101) \\
(Pig)
\end{tabular} & $\begin{array}{l}\text { RCS; } \\
\text { resuscitation } \\
\text { model }\end{array}$ & \begin{tabular}{|l|} 
Bupivacaine \\
$(2 \mathrm{mg} / \mathrm{kg} / \mathrm{min})$ or \\
Mepivacaine \\
$(6 \mathrm{mg} / \mathrm{kg} / \mathrm{min})$ \\
\end{tabular} & 2.68 & $\begin{array}{l}\text { MAP decreased } \\
\text { to } 50 \% \text { of its } \\
\text { baseline value }\end{array}$ & $\begin{array}{l}20 \% \\
\text { ClinOleic } \\
\text { and } \\
\text { Intralipid }\end{array}$ & \begin{tabular}{|l}
$1.5 \mathrm{~mL} / \mathrm{kg}$ \\
$(0.3 \mathrm{~g} / \mathrm{kg})$
\end{tabular} & $\begin{array}{l}0.25 \mathrm{~mL} / \mathrm{kg} \\
/ \mathrm{min}(3 \\
\mathrm{g} / \mathrm{kg} / \mathrm{hr}) \text { for } \\
29 \mathrm{~min}\end{array}$ & \begin{tabular}{|l} 
ILE \\
vs \\
Ringer acetate
\end{tabular} & $\begin{array}{l}\text { Other } \\
\text { treatment and } \\
\text { study treatment } \\
\text { immediately } \\
\text { after LA }\end{array}$ & $\begin{array}{l}\text { EPI } 0.5 \mathrm{mg} \\
\text { repeated } \\
\text { doses, chest } \\
\text { compression, } \\
\text { mechanical } \\
\text { ventilation, } \\
\text { electrical } \\
\text { defibrillation. }\end{array}$ & $\begin{array}{l}\text { Hemodynamics: } \\
\text { Comparison of MAP and HR } \\
\text { among treatment groups at } \\
\text { each measured time point } \\
\text { revealed no overall effect } \\
\text { of ILE in comparison with } \\
\text { Ringer's acetate solution; } \\
\text { No difference in effect } \\
\text { between the two ILE's } \\
\text { tested } \\
\end{array}$ & \begin{tabular}{|l|} 
Survival: Bupi+ILE \\
10/10, \\
Bupi+Ringer $8 / 10$, \\
Mepi+ILE $9 / 10$, \\
Mepi+Ringer 10/10 \\
\end{tabular} & $\begin{array}{l}\text { No; ILEs did not } \\
\text { improve hemodynamic } \\
\text { parameters }\end{array}$ \\
\hline \begin{tabular}{|l|}
$\begin{array}{l}\text { Mauch, } 2012(102) \\
\text { (Pig) }\end{array}$ \\
\end{tabular} & $\begin{array}{l}\text { RCS; } \\
\text { compared ILE } \\
\text { effect with EPI } \\
\text { and vasopressin }\end{array}$ & $\begin{array}{l}\text { Bupivacaine } \\
(1 \mathrm{mg} / \mathrm{kg} / \mathrm{min})\end{array}$ & 2.68 & Cardiac arrest & \begin{tabular}{|l}
$20 \%$ \\
Intralipid
\end{tabular} & \begin{tabular}{|l}
$4 \mathrm{~mL} / \mathrm{kg}$ \\
$(0.8 \mathrm{~g} / \mathrm{kg})$
\end{tabular} & No & \begin{tabular}{|l} 
ILE \\
vs \\
ILE+EPI \\
vs \\
ILE+VASO \\
vs \\
EPI
\end{tabular} & \begin{tabular}{l|l} 
Other \\
treatment \\
immediately \\
after LA, study \\
treatment at 1 \\
min.
\end{tabular} & $\begin{array}{l}\text { Vasopressors } \\
2 \mathrm{IU}, \mathrm{EPI} 10 \\
\mathrm{ug} / \mathrm{kg} \\
\text { chest } \\
\text { compression }\end{array}$ & \begin{tabular}{|l|} 
ROSC was regained after \\
one EPI rescue dose in EPI \\
and EPI+ILE. \\
ILE and VASO+ILE ROSC \\
achieved after secondary \\
EPI rescue dose
\end{tabular} & $\begin{array}{l}\text { Survival: ILE 2/7, } \\
\text { ILE+EPI 6/7 } \\
\text { ILEVVASO 4/7, } \\
\text { EPI 5/7 }\end{array}$ & $\begin{array}{l}\text { No; Supports using EPI } \\
\text { and EPI+ILE } \\
\text { resuscitation over ILE } \\
\text { resuscitation alone and } \\
\text { ILE+VASO }\end{array}$ \\
\hline \begin{tabular}{|l|}
$\begin{array}{l}\text { Mauch, } 2011(103) \\
\text { (Pig) }\end{array}$ \\
\end{tabular} & $\begin{array}{l}\text { RCS; } \\
\text { compare } \\
\text { effectiveness of } \\
\text { EPI and ILE }\end{array}$ & \begin{tabular}{|l|} 
Bupivacaine \\
$(1 \mathrm{mg} / \mathrm{kg} / \mathrm{min}$ \\
until symptoms $)$
\end{tabular} & 2.68 & \begin{tabular}{|l} 
LA infused at a \\
rate of 1 \\
$\mathrm{mg} / \mathrm{kg}^{*}$ min until \\
invasively \\
measured MAP \\
dropped to $50 \%$ \\
of the initial \\
value
\end{tabular} & $\begin{array}{l}20 \% \\
\text { Intralipid }\end{array}$ & $\begin{array}{l}2 \mathrm{~mL} / \mathrm{kg} \\
(0.4 \mathrm{~g} / \mathrm{kg}) \\
\text { and } \\
4 \mathrm{~mL} / \mathrm{kg} \\
(0.8 \mathrm{~g} / \mathrm{kg})\end{array}$ & No & $\begin{array}{l}\text { ILE } 2 \mathrm{ml} / \mathrm{kg} \\
\text { vs } \\
\text { ILE } 4 \mathrm{ml} / \mathrm{kg} \\
\text { vs } \\
\text { EPI }\end{array}$ & $\begin{array}{l}\text { Other } \\
\text { treatment and } \\
\text { study treatment } \\
\text { immediately } \\
\text { after LA }\end{array}$ & \begin{tabular}{|l|} 
EPI rescue \\
doses, 3 \\
$\mathrm{mcg} / \mathrm{kg}$, every \\
$5 \mathrm{~min}$ if \\
$\mathrm{MAP}<75 \%$
\end{tabular} & 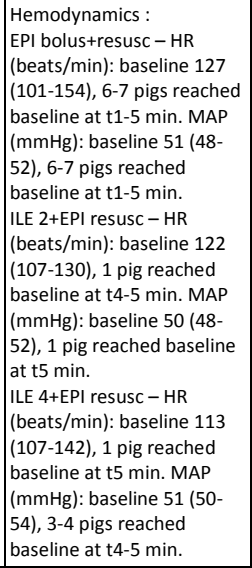 & $\begin{array}{l}\text { Survival: ILE 2+EPI } \\
\text { resusc } 4 / 7, \\
\text { ILE } 4+\text { EPI resusc } \\
4 / 7 \\
\text { EPI bolus+resusc } \\
7 / 7,\end{array}$ & $\begin{array}{l}\text { Yes; But EPI may be } \\
\text { better first line therapy }\end{array}$ \\
\hline
\end{tabular}


AACT manuscript LA to edit for CTX

\begin{tabular}{|c|c|c|c|c|c|c|c|c|c|c|c|c|c|}
\hline $\begin{array}{l}\text { Reference } \\
\text { (Species) }\end{array}$ & Model & $\begin{array}{l}\text { Local } \\
\text { anesthetic } \\
\text { (dose) }\end{array}$ & $\left.\mid \begin{array}{l}\text { Log } \\
D \\
(129)\end{array}\right)$ & Symptoms & $\begin{array}{l}\text { ILE } \\
\text { used }\end{array}$ & \begin{tabular}{|l} 
ILE \\
bolus \#
\end{tabular} & $\begin{array}{l}\text { ILE } \\
\text { infusion } \\
\#\end{array}$ & Study arms & \begin{tabular}{|l|} 
Timing of \\
rescue, \\
time from \\
LA \\
termination
\end{tabular} & $\begin{array}{l}\text { Other } \\
\text { treatments } \\
\text { received * }\end{array}$ & $\begin{array}{l}\text { Parameter } \\
\text { measured }\end{array}$ & Outcome & $\begin{array}{l}\text { Support } \\
\text { therapeutic } \\
\text { effect of ILE } \\
\text { alone }\end{array}$ \\
\hline $\begin{array}{l}\text { Mauch, } 2011 \\
\text { (104) ฉ } \\
\text { (Pig) }\end{array}$ & $\begin{array}{l}\text { RCS; } \\
\text { resuscitation } \\
\text { model }\end{array}$ & \begin{tabular}{|l} 
Bupivacaine \\
$(1 \mathrm{mg} / \mathrm{kg} / \mathrm{min}$ \\
until symptoms $)$
\end{tabular} & 2.68 & \begin{tabular}{|l} 
Pulseless \\
electrical activity, \\
$n=23$ \\
Asstole, $n=2$. \\
LA infused until \\
cardiac arrest \\
(pulseless \\
electrical activity \\
was defined as \\
MAP 25\% of \\
initial value, \\
corresponding to \\
$12-13 \mathrm{mmHg}$ ).
\end{tabular} & Intralipid & \begin{tabular}{|l}
$4 \mathrm{~mL} / \mathrm{kg}$ \\
$(0.8 \mathrm{~g} / \mathrm{kg})$
\end{tabular} & No & \begin{tabular}{|l} 
ILE \\
vs \\
EPI \\
vs \\
ILE+EPI \\
vs \\
ILE+VASO
\end{tabular} & \begin{tabular}{|l} 
Other \\
treatment \\
immediately \\
after LA, study \\
treatment at 1 \\
min.
\end{tabular} & \begin{tabular}{|l|} 
EPI bolus 10 \\
mcg/kg. \\
Followed by \\
rescue doses \\
every 5 min if \\
necessary, 10 \\
mcg/kg in case \\
of cardiac \\
arrest, or 3 \\
mcg//kg if \\
MAP $575 \%$, \\
chest \\
compressions, \\
mechanical \\
ventilation
\end{tabular} & \begin{tabular}{l|} 
Hemodynamics: EPI+std \\
resusc - Secondary high \\
dose EPI was not needed in \\
surviving pigs; low dose EPI \\
for haemodynamic support \\
was given in 3 of 5 \\
surviving pigs; \\
ILE - Secondary high dose \\
EPI was needed in all \\
surviving pigs; low dose EPI \\
for haemodynamic support \\
was given in 1 surviving pig; \\
ILEEEPI - Secondary high \\
dose EP was not needed in \\
surviving pigs; \\
ILE+VASO - Secondary high \\
dose EPI was needed in all \\
surviving pigs
\end{tabular} & $\begin{array}{l}\text { Survival:ILE 1/6, } \\
\text { EPI 5/7, } \\
\text { ILE+EPI 5/6, } \\
\text { ILE+VASO } 3 / 6 \\
\\
\end{array}$ & $\begin{array}{l}\text { No; EPI and EPI+ILE } \\
\text { resuscitation was } \\
\text { superior to ILE alone }\end{array}$ \\
\hline \begin{tabular}{|l|}
$\begin{array}{l}\text { Mayr, } 2008 \text { (105) } \\
\text { (Pig) }\end{array}$ \\
\end{tabular} & \begin{tabular}{|l} 
RCS; \\
compare \\
resuscitation \\
with ILE and \\
VASO/EPI
\end{tabular} & \begin{tabular}{|l|} 
Bupivacaine \\
(5 mg/kg)
\end{tabular} & 2.68 & \begin{tabular}{|l|} 
Aortic blood \\
pressure \\
decreasing to \\
hydrostatic \\
pressure; \\
Asystole
\end{tabular} & \begin{tabular}{|l|}
$20 \%$ \\
Intralipid
\end{tabular} & \begin{tabular}{|l|}
$4 \mathrm{~mL} / \mathrm{kg}$ \\
$(0.8 \mathrm{~g} / \mathrm{kg})$
\end{tabular} & $\begin{array}{l}0.5 \\
\mathrm{~mL} / \mathrm{kg} / \mathrm{min} \\
(6 \mathrm{~g} / \mathrm{kg} / \mathrm{hr}) \\
\text { for } 10 \mathrm{~min}\end{array}$ & \begin{tabular}{|l|} 
ILE \\
vs \\
VASO+EPI
\end{tabular} & $\begin{array}{l}\text { Other } \\
\text { treatment at } 1 \\
\text { min., study } \\
\text { treatment at } 2 \\
\text { min. }\end{array}$ & \begin{tabular}{|l|} 
Ringer's \\
solution, \\
gelatin \\
solution, saline \\
(one group); \\
VASO, EPI \\
(saline group); \\
Azaperone 4 \\
mg/kg IM, \\
atropine 0.1 \\
mg/kg IM; \\
Anesthesia, \\
ketamine 20 \\
mg//g IM, \\
piritramid 30 \\
mg IV, \\
maintained \\
with isoflurane \\
1-2\% end-tidal; \\
Heparin; \\
Oxygen \\
\end{tabular} & \begin{tabular}{|l|} 
Hemodynamics: \\
ILE+Saline - none of the ILE- \\
pigs had restoration of \\
spontaneous circulation, \\
coronary perfusion \\
pressure <20-30 mmHg. \\
EPIIVASO+Saline - \\
coronary perfusion \\
pressure as a decisive \\
predictor of spontaneous \\
circulation was significantly \\
higher $90 \mathrm{~s}$ after the first \\
and second VASO/EPI \\
injection compared to ILE, \\
$220-30 \mathrm{mmHg}$
\end{tabular} & $\begin{array}{l}\text { Survival: ILE+Saline } \\
\text { O/5, } \\
\text { EPI+VASO+Saline } \\
5 / 5\end{array}$ & $\begin{array}{l}\text { No; Supports use of } \\
\text { Vasopressors+EPI over } \\
\text { ILE resuscitation }\end{array}$ \\
\hline
\end{tabular}


AACT manuscript LA to edit for CTX

\begin{tabular}{|c|c|c|c|c|c|c|c|c|c|c|c|c|c|}
\hline $\begin{array}{l}\text { Reference } \\
\text { (Species) }\end{array}$ & Model & \begin{tabular}{|l} 
Local \\
anesthetic \\
(dose)
\end{tabular} & \begin{tabular}{|l|} 
Log \\
$D$ \\
$(129)$
\end{tabular} & Symptoms & $\begin{array}{l}\text { ILE } \\
\text { used }\end{array}$ & \begin{tabular}{|l|} 
ILE \\
bolus \#
\end{tabular} & \begin{tabular}{|l} 
ILE \\
infusion \\
$\#$
\end{tabular} & Study arms & \begin{tabular}{|l|} 
Timing of \\
rescue, \\
time from \\
LA \\
termination
\end{tabular} & \begin{tabular}{|l|} 
Other \\
treatments \\
received *
\end{tabular} & $\begin{array}{l}\text { Parameter } \\
\text { measured }\end{array}$ & Outcome & \begin{tabular}{|l} 
Support \\
therapeutic \\
effect of ILE \\
alone
\end{tabular} \\
\hline $\begin{array}{l}\text { Shi, } 2003 \text { (106) } \\
\text { (Rats) }\end{array}$ & \begin{tabular}{|l|} 
RCS; \\
Evaluation of the \\
effect on \\
hemodynamics \\
and LA \\
pharmacokinetics
\end{tabular} & \begin{tabular}{|l|} 
Bupivacaine \\
$(2 \mathrm{mg} / \mathrm{kg} / \mathrm{min}$ for \\
$4 \mathrm{~min})$
\end{tabular} & 2.68 & $\begin{array}{l}\begin{array}{l}\text { Hypotension, } \\
\text { bradycardia }\end{array} \\
\end{array}$ & \begin{tabular}{|l|}
$30 \%$, \\
Intralipid
\end{tabular} & No & $\begin{array}{l}3 \mathrm{ml} / \mathrm{kg} / \mathrm{min} \\
(54 \mathrm{~g} / \mathrm{kg} / \mathrm{hr} \\
\text { for } 5 \mathrm{~min})\end{array}$ & \begin{tabular}{|l} 
ILE \\
Vs \\
Saline
\end{tabular} & \begin{tabular}{|l|}
$\begin{array}{l}\text { Study } \\
\text { treatment or } \\
\text { other treatment } \\
\text { immediately } \\
\text { after LA }\end{array}$ \\
\end{tabular} & No & \begin{tabular}{|l|} 
HR, MAP: Comparable \\
Plasma-bupivacaine: \\
distribution constant, \\
elimination half-life(t1/2) \\
decreased in ILE grouss, \\
elimination half-life(t1/2a), \\
clearance increased in ILE \\
groups. \\
Bupivacaine tissue (brain, \\
myocytes, lung, kidney, \\
spleen, muscle) content \\
reduced in ILE group; \\
increased in liver ILE group \\
compared to saline
\end{tabular} & Survival: All & $\begin{array}{l}\text { Yes; ILE accelerated the } \\
\text { elimination of } \\
\text { bupivacaine. The lipid } \\
\text { sink phenomenon was } \\
\text { observed. }\end{array}$ \\
\hline $\begin{array}{l}\text { Wat, } 2009(107) x \\
\text { (Pig) }\end{array}$ & $\begin{array}{l}\text { RCS; } \\
\text { resuscitation } \\
\text { model }\end{array}$ & \begin{tabular}{|l|} 
Ropivacaine \\
$(14.9 \pm 2.8 \mathrm{mg} / \mathrm{kg})$
\end{tabular} & 4.21 & $\begin{array}{l}\text { Cardiovascular } \\
\text { collapse }\end{array}$ & \begin{tabular}{|l|}
$20 \%$, \\
Intralipid
\end{tabular} & \begin{tabular}{|l|}
$4 \mathrm{~mL} / \mathrm{kg}$ \\
$(0.8 \mathrm{~g} / \mathrm{kg})$ \\
\end{tabular} & $\begin{array}{l}0.5 \\
\mathrm{~mL} / \mathrm{kg} / \mathrm{min} \\
(6 \mathrm{~g} / \mathrm{kg} / \mathrm{hr})\end{array}$ & \begin{tabular}{|l} 
ILE \\
vs \\
EPI \\
vs \\
Saline
\end{tabular} & \begin{tabular}{|l|l} 
Other & \\
treatment \\
immediately, \\
study treatment \\
NR
\end{tabular} & $\begin{array}{l}\text { Cardiac } \\
\text { massage }\end{array}$ & \begin{tabular}{|l|} 
Failed to regain $50 \%$ of \\
baseline systolic blood \\
pressure and HR $10 \mathrm{~min}$ \\
after iv treatment \\
commenced. \\
myocardial ATP content \\
were not different between \\
groups
\end{tabular} & \begin{tabular}{|l} 
Survival: ILE 0/5, \\
Epi 5/5, \\
Saline 0/5
\end{tabular} & $\begin{array}{l}\text { No; Ropivacaine } \\
\text { induced cardiac toxicity } \\
\text { responded well to } \\
\text { standard resuscitation } \\
\text { with cardiac massage } \\
\text { and intravenous } \\
\text { adrenaline }\end{array}$ \\
\hline $\begin{array}{l}\text { Weinberg, } 2004 \\
\text { (108) } \\
\text { (Dogs) }\end{array}$ & \begin{tabular}{|l|} 
RCS; \\
evaluation of the \\
effect of \\
bupivacaine on \\
myocardial \\
acidosis induced \\
by ventricular \\
fibrillation \\
\end{tabular} & $\begin{array}{l}\text { Bupivacaine } \\
(10 \mathrm{mg} / \mathrm{kg})\end{array}$ & 2.68 & $\begin{array}{l}\text { Ventricular } \\
\text { fibrillation or } \\
\text { myocardial pH } \\
7.0\end{array}$ & \begin{tabular}{|l|}
$20 \%$, \\
Intralipid
\end{tabular} & \begin{tabular}{|l|}
$4 \mathrm{~mL} / \mathrm{kg}$ \\
$(0.8 \mathrm{~g} / \mathrm{kg})$ \\
\end{tabular} & $\begin{array}{l}0.5 \\
\mathrm{~mL} / \mathrm{kg} / \mathrm{min} \\
(6 \mathrm{~g} / \mathrm{kg} / \mathrm{hr}) \\
\text { for } 10 \mathrm{~min}\end{array}$ & \begin{tabular}{|l|} 
LA+ILE \\
vs \\
Saline+Fibrillation \\
(no LA, fibrillation \\
until same \\
symptoms as LA \\
group)
\end{tabular} & \begin{tabular}{l|l} 
Defibrillation at \\
20 min or at \\
myocardial \\
pH $\mathbf{3} .0$
\end{tabular} & $\begin{array}{l}\text { Fibrillation in } \\
\text { non-ILE group }\end{array}$ & \begin{tabular}{|l|} 
PmO2 was comparable in \\
saline vs LA+LLE group; \\
Tissue pH decreased 4 \\
times faster in saline \\
compared to LA group \\
during ventricular \\
fibrillation; \\
Time to normal sinus \\
rhythm was comparable in \\
Saline vs LA+ILE group \\
\end{tabular} & $\begin{array}{l}\text { Survival: LA+ILE } \\
8 / 8, \\
\text { Saline } 8 / 8\end{array}$ & Not studied \\
\hline $\begin{array}{l}\text { Weinberg, } 2003 \\
\text { (109) } \\
\text { (Dogs) }\end{array}$ & \begin{tabular}{|l|} 
RCS; \\
resuscitation \\
model
\end{tabular} & $\begin{array}{l}\text { Bupivacaine } \\
(10 \mathrm{mg} / \mathrm{kg} \text { in } 10 \\
\mathrm{sec})\end{array}$ & 2.68 & Cardiac arrest & \begin{tabular}{|l|}
$20 \%$, \\
Intralipid
\end{tabular} & \begin{tabular}{|l|}
$4 \mathrm{~mL} / \mathrm{kg}$ \\
$(0.8 \mathrm{~g} / \mathrm{kg})$ \\
\end{tabular} & \begin{tabular}{|l|}
0.5 \\
$\mathrm{~mL} / \mathrm{kg} / \mathrm{min}$ \\
$6 \mathrm{~g} / \mathrm{kg} / \mathrm{hr})$ \\
for $10 \mathrm{~min}$
\end{tabular} & \begin{tabular}{|l} 
ILE \\
vs \\
Saline
\end{tabular} & \begin{tabular}{|l|l} 
Other \\
treatment \\
immediately, \\
study treatment \\
at 10 min.
\end{tabular} & \begin{tabular}{|l|} 
Mechanical \\
ventilation, \\
internal cardiac \\
massage
\end{tabular} & \begin{tabular}{|l|} 
Hemodynamics: PmO2, and \\
pHm were improved during \\
resuscitation with ILE \\
compared with saline \\
treatment in which dogs \\
did not recover. Data are \\
compared between \\
baseline and after recovery
\end{tabular} & $\begin{array}{l}\text { Survival: ILE 6/6, } \\
\text { Saline 0/6 }\end{array}$ & Yes \\
\hline
\end{tabular}


AACT manuscript LA to edit for CTX

\begin{tabular}{|c|c|c|c|c|c|c|c|c|c|c|c|c|c|}
\hline $\begin{array}{l}\text { Reference } \\
\text { (Species) }\end{array}$ & Model & $\begin{array}{l}\text { Local } \\
\text { anesthetic } \\
\text { (dose) }\end{array}$ & \begin{tabular}{|l} 
Log \\
$D$ \\
$(129)$
\end{tabular} & Symptoms & $\begin{array}{l}\text { ILE } \\
\text { used }\end{array}$ & \begin{tabular}{|l} 
ILE \\
bolus \#
\end{tabular} & \begin{tabular}{|l} 
ILE \\
infusion \\
$\#$
\end{tabular} & Study arms & \begin{tabular}{|l|} 
Timing of \\
rescue, \\
time from \\
LA \\
termination
\end{tabular} & $\begin{array}{l}\text { Other } \\
\text { treatments } \\
\text { received * }\end{array}$ & $\begin{array}{l}\text { Parameter } \\
\text { measured }\end{array}$ & Outcome & $\begin{array}{l}\text { Support } \\
\text { therapeutic } \\
\text { effect of ILE } \\
\text { alone }\end{array}$ \\
\hline \begin{tabular}{|l} 
Weinberg, 2008 \\
(110) \\
(Rats)
\end{tabular} & $\begin{array}{l}\text { RCS; } \\
\text { resuscitation } \\
\text { model }\end{array}$ & \begin{tabular}{|l} 
Bupivacaine \\
$(20 \mathrm{mg} / \mathrm{kg})$
\end{tabular} & 2.68 & Asystole & \begin{tabular}{|l|}
$30 \%$ \\
Intralipid
\end{tabular} & \begin{tabular}{|l|}
$5 \mathrm{~mL} / \mathrm{kg}$ \\
$(1.5 \mathrm{~g} / \mathrm{kg})$
\end{tabular} & $\begin{array}{l}0.5 \\
\mathrm{~mL} / \mathrm{kg} / \mathrm{min} \\
(9 \mathrm{~g} / \mathrm{kg} / \mathrm{hr})\end{array}$ & \begin{tabular}{|l} 
ILE \\
vs \\
EPI \\
vs \\
Saline
\end{tabular} & \begin{tabular}{|l|}
$\begin{array}{l}\text { Other } \\
\text { treatment and } \\
\text { study treatment } \\
\text { immediately } \\
\text { after LA }\end{array} \mid$ \\
\end{tabular} & \begin{tabular}{|l|} 
Chest \\
compressions, \\
mechanical \\
ventilation
\end{tabular} & $\begin{array}{l}\text { QRS width: } \\
\text { ILE - comparative } \\
\text { EPI - Significantly } \\
\text { prolonged at } 2.5 \mathrm{~min} \\
\text { compared to ILE; But } \\
\text { shorter than saline control. } \\
\text { recovered to baseline at } 5 \\
\text { min. } \\
\text { Saline - Significantly } \\
\text { prolonged at } 2.5 \text { min } \\
\text { compared to ILE, stayed } \\
\text { elevated throughout the } \\
\text { experiment }\end{array}$ & $\begin{array}{l}\text { Survival: ILE 5/5, } \\
\text { EPI 5/5, } \\
\text { Saline 5/5 }\end{array}$ & Yes \\
\hline \begin{tabular}{|l|} 
Yan, 2012 (111) \\
(Rats)
\end{tabular} & $\begin{array}{l}\text { RCS; } \\
\text { resuscitation } \\
\text { model }\end{array}$ & \begin{tabular}{|l|} 
Bupivacaine \\
(30 mg/kg)
\end{tabular} & 2.68 & Asystole & \begin{tabular}{|l|}
$20 \%$ \\
Intralipid
\end{tabular} & $\begin{array}{l}5 \mathrm{~mL} / \mathrm{kg}(1 \\
\mathrm{g} / \mathrm{kg})\end{array}$ & \begin{tabular}{|l}
0.5 \\
$\mathrm{~mL} / \mathrm{kg} / \mathrm{min}$ \\
$(6 \mathrm{~g} / \mathrm{kg} / \mathrm{hr})$
\end{tabular} & \begin{tabular}{|l|} 
ILE \\
vs \\
ILE+EPI \\
vs \\
EPI \\
vs \\
Saline
\end{tabular} & \begin{tabular}{|l|}
$\begin{array}{l}\text { Other } \\
\text { treatment } \\
\text { immediately, } \\
\text { study treatment } \\
\text { at 10 min. }\end{array}$ \\
\end{tabular} & No & $\begin{array}{l}\text { Hemodynamic parameters } \\
\text { at 25min., coronary } \\
\text { perfusion. Post-mortem } \\
\text { myocardial LA content }\end{array}$ & $\begin{array}{l}\text { Survival: ILE } 3 / 8, \\
\text { EPI+LLE } 5 / 8, \\
\text { EPI } 2 / 8, \\
\text { Saline } 0 / 8\end{array}$ & \begin{tabular}{|l|} 
No; EPI+LEE had \\
improved \\
hemodynamics \\
compared to ILE alone
\end{tabular} \\
\hline \multicolumn{14}{|l|}{\begin{tabular}{|l|} 
Observational \\
\end{tabular}} \\
\hline \begin{tabular}{|l|} 
Callejo, 2014 (113) \\
$x$ \\
(Pig)
\end{tabular} & 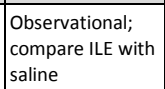 & $\begin{array}{l}\text { Bupivacaine } \\
\text { (4 mg/kg) }\end{array}$ & 2.68 & $\begin{array}{l}\text { 150\% increase in } \\
\text { QRS duration }\end{array}$ & \begin{tabular}{|l|} 
Conc. NR, \\
Intralipid
\end{tabular} & $1.5 \mathrm{~mL} / \mathrm{kg}$ & $\begin{array}{l}0.25 \\
\mathrm{~mL} / \mathrm{kg} / \mathrm{min}\end{array}$ & \begin{tabular}{|l|} 
ILE \\
vs \\
Saline
\end{tabular} & NR & NR & $\begin{array}{l}\text { QRS widening was reversed } \\
\text { after ILE }\end{array}$ & $\begin{array}{l}\text { Survival NR. ILE: } \\
? / 6 \text {, Saline ?/3 }\end{array}$ & \begin{tabular}{|l|} 
No; Suggests \\
concomitant \\
resuscitation measures
\end{tabular} \\
\hline
\end{tabular}


AACT manuscript LA to edit for CTX

\begin{tabular}{|c|c|c|c|c|c|c|c|c|c|c|c|c|c|}
\hline $\begin{array}{l}\text { Reference } \\
\text { (Species) }\end{array}$ & Model & \begin{tabular}{|l} 
Local \\
anesthetic \\
(dose)
\end{tabular} & $\left.\mid \begin{array}{l}\text { Log } \\
D \\
(129)\end{array}\right)$ & Symptoms & $\begin{array}{l}\text { ILE } \\
\text { used }\end{array}$ & \begin{tabular}{|l} 
ILE \\
bolus \#
\end{tabular} & \begin{tabular}{|l|} 
ILE \\
infusion \\
$\#$
\end{tabular} & Study arms & \begin{tabular}{|l|} 
Timing of \\
rescue, \\
time from \\
LA \\
termination
\end{tabular} & $\begin{array}{l}\text { Other } \\
\text { treatments } \\
\text { received * }\end{array}$ & $\begin{array}{l}\text { Parameter } \\
\text { measured }\end{array}$ & Outcome & $\begin{array}{l}\text { Support } \\
\text { therapeutic } \\
\text { effect of ILE } \\
\text { alone }\end{array}$ \\
\hline $\begin{array}{l}\text { Cave, } 2010 \text { (114) } \\
\text { (Rabbit) }\end{array}$ & \begin{tabular}{|l|} 
Observational; \\
compared to 21\% \\
saline
\end{tabular} & $\begin{array}{l}\text { Bupivacaine } \\
(10 \mathrm{mg} / \mathrm{kg})\end{array}$ & 2.68 & Asystole & \begin{tabular}{|l}
$20 \%$ \\
Intralipid
\end{tabular} & \begin{tabular}{|l|}
$1.5 \mathrm{~mL} / \mathrm{kg}$ \\
$(0.3 \mathrm{~g} / \mathrm{kg})$
\end{tabular} & \begin{tabular}{|l|}
0.25 \\
$\mathrm{~mL} / \mathrm{kg} / \mathrm{min}$ \\
$(3 \mathrm{~g} / \mathrm{kg} / \mathrm{hr})$
\end{tabular} & \begin{tabular}{|l} 
ILE \\
vs \\
ILE+Hypertonic \\
saline (21\%)
\end{tabular} & \begin{tabular}{|l|} 
Other \\
treatment at 30 \\
sec., study \\
treatment at 1 \\
min.
\end{tabular} & \begin{tabular}{|l|} 
Hypertonic \\
saline (one \\
group); \\
Adrenaline \\
ketamine \\
Chest \\
compressions \\
and mechanical \\
ventilation, \\
with 100\% \\
oxygen.
\end{tabular} & 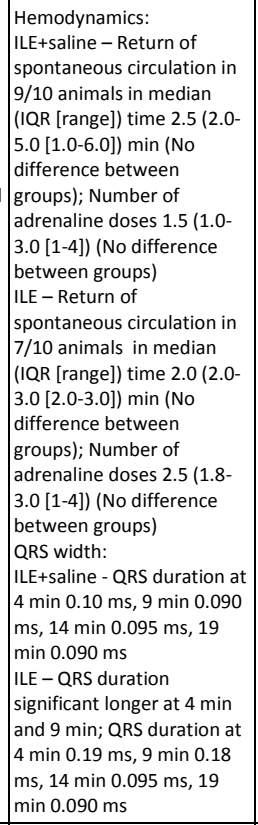 & $\begin{array}{l}\text { Survival: ILE 5/10, } \\
\text { ILE+21\% saline } \\
6 / 10\end{array}$ & Not studied \\
\hline $\begin{array}{l}\text { Weinberg, } 1998 \\
\text { (115) } \\
\text { (Rats) }\end{array}$ & $\begin{array}{l}\text { Observational; } \\
\text { resuscitation } \\
\text { model }\end{array}$ & \begin{tabular}{|l} 
Bupivacaine \\
(various dose)
\end{tabular} & 2.68 & Asystole & \begin{tabular}{|l|}
$30 \%$ \\
Intralipid
\end{tabular} & $\begin{array}{l}7.5 \mathrm{ml} / \mathrm{kg} \\
(2.25 \mathrm{~g} / \mathrm{kg})\end{array}$ & \begin{tabular}{|l|}
3 \\
$\mathrm{~mL} / \mathrm{kg} / \mathrm{min}$ \\
$(54 \mathrm{~g} / \mathrm{kg} / \mathrm{hr})$ \\
for $2 \mathrm{~min}$ \\
Total dose 4 \\
$\mathrm{~g} / \mathrm{kg}$
\end{tabular} & \begin{tabular}{|l|} 
ILE \\
vs \\
Saline
\end{tabular} & \begin{tabular}{|l|}
$\begin{array}{l}\text { Other } \\
\text { treatment and } \\
\text { study treatment } \\
\text { immediately } \\
\text { after LA }\end{array}$ \\
\end{tabular} & \begin{tabular}{|l|} 
Bicarbonate: 2 \\
min \\
IV fluids: 2.5 \\
min \\
Vasopressors: \\
$3.05 \mathrm{~g} / \mathrm{kg}$ \\
\end{tabular} & \begin{tabular}{|l|} 
ILE increased the dose \\
required to cause death in \\
$50 \%$ of animals
\end{tabular} & LD50 study & $\begin{array}{l}\text { Yes; Increase LD50 } \\
\text { dose from } 12.5 \text { to } 18.5 \\
\mathrm{mg} / \mathrm{kg}\end{array}$ \\
\hline Case Series & & & & & & & & & & & & & \\
\hline
\end{tabular}


AACT manuscript LA to edit for CTX

\begin{tabular}{|c|c|c|c|c|c|c|c|c|c|c|c|c|c|}
\hline $\begin{array}{l}\text { Reference } \\
\text { (Species) }\end{array}$ & Model & $\begin{array}{l}\text { Local } \\
\text { anesthetic } \\
\text { (dose) }\end{array}$ & $\begin{array}{l}\text { Log } \\
D \\
(129)\end{array}$ & Symptoms & $\begin{array}{l}\text { ILE } \\
\text { used }\end{array}$ & \begin{tabular}{|l} 
ILE \\
bolus \#
\end{tabular} & \begin{tabular}{|l} 
ILE \\
infusion \\
$\#$
\end{tabular} & Study arms & \begin{tabular}{|l|} 
Timing of \\
rescue, \\
time from \\
LA \\
termination
\end{tabular} & \begin{tabular}{|l|} 
Other \\
treatments \\
received *
\end{tabular} & $\begin{array}{l}\text { Parameter } \\
\text { measured }\end{array}$ & Outcome & $\begin{array}{l}\text { Support } \\
\text { therapeutic } \\
\text { effect of ILE } \\
\text { alone }\end{array}$ \\
\hline \begin{tabular}{|l|} 
Harvey, 2010 \\
$(116)$ \\
(Rabbit)
\end{tabular} & $\begin{array}{l}\text { Case series; } \\
\text { resuscitation } \\
\text { model }\end{array}$ & \begin{tabular}{|l|}
$\begin{array}{l}\text { Bupivacaine } \\
(10 \mathrm{~m} / \mathrm{kg})\end{array}$ \\
\end{tabular} & 2.68 & Asystole & $\begin{array}{l}20 \% \\
\text { Intralipid }\end{array}$ & $\begin{array}{l}5 \mathrm{~mL} / / \mathrm{kg}(1 \\
\mathrm{g} / \mathrm{kg}) \times 2\end{array}$ & No & \begin{tabular}{|l|} 
ILE \\
vs \\
ILE+EPI
\end{tabular} & \begin{tabular}{|l|} 
Other \\
treatment at 30 \\
sec., study \\
treatment at 1 \\
min.
\end{tabular} & \begin{tabular}{|l|} 
Control group \\
received late \\
dosing of EPI \\
$100 \mathrm{mcg} / \mathrm{kg} \mathrm{(1}$ \\
$\mathrm{mL} / \mathrm{kg})$
\end{tabular} & \begin{tabular}{|l} 
Hemodynamics: Time to \\
ROSC after chest \\
compressions and \\
mechanical ventilation. \\
High-dose EPI \\
administration was \\
associated with a \\
significant increase in \\
coronary perfusion \\
pressure before ROSC
\end{tabular} & \begin{tabular}{|l} 
Survival: None; \\
Failure of animals \\
from any group to \\
maintain effective \\
circulation
\end{tabular} & \begin{tabular}{|l} 
No; ILE alone was \\
compared to ILE with 3 \\
doses of EPI. Only high \\
dose EPI+ILE had ROSC
\end{tabular} \\
\hline \begin{tabular}{|l}
$\begin{array}{l}\text { Partownavid } 2012 \\
\text { (p.2431) (117) } \\
\text { (Rats) }\end{array}$ \\
\end{tabular} & $\begin{array}{l}\text { Case series; } \\
\text { resuscitation } \\
\text { model }\end{array}$ & $\begin{array}{l}\text { Bupivacaine } \\
(10 \mathrm{mg} / \mathrm{kg})\end{array}$ & 2.68 & Asystole & NR & $5 \mathrm{~mL} / \mathrm{kg}$ & $\begin{array}{l}0.5 \\
\mathrm{~mL} / \mathrm{kg} / \mathrm{min}\end{array}$ & \begin{tabular}{|l|} 
ILE \\
vs \\
Pre-treatment+ILE
\end{tabular} & \begin{tabular}{|l} 
Other \\
treatment \\
immediately
\end{tabular} & \begin{tabular}{|l|} 
Pre-treatment \\
with naloxone \\
$1 \mathrm{mg} / \mathrm{kg}$, \\
$10 \mathrm{mcg} / \mathrm{kg}$, \\
$5 \mathrm{mcg} / \mathrm{kg}$, \\
$1 \mathrm{mcg} / \mathrm{kg}$
\end{tabular} & $\begin{array}{l}\mathrm{HR} \text {, ejection fraction and } \\
\text { fractional shortening } \\
\text { measures by ECG at } \\
\text { baseline, } 1,5,10 \mathrm{~min} .\end{array}$ & $\begin{array}{l}\text { Survival rate } \\
\text { unclear }\end{array}$ & $\begin{array}{l}\text { Not studied; Naloxone } \\
\text { abolishes ILE rescue of } \\
\text { bupivacaine-induced } \\
\text { cardiotoxicity in a dose } \\
\text { dependent manner }\end{array}$ \\
\hline \begin{tabular}{|l}
$\begin{array}{l}\text { Partownavid } 2010 \\
(118) x \\
\text { (Rats) }\end{array}$ \\
\end{tabular} & $\begin{array}{l}\text { Case series; } \\
\text { resuscitation } \\
\text { model }\end{array}$ & $\begin{array}{l}\text { Bupivacaine } \\
(10 \mathrm{mg} / \mathrm{kg})\end{array}$ & 2.68 & Asystole & \begin{tabular}{|l|}
$20 \%$ \\
Intralipid
\end{tabular} & $\begin{array}{l}5 \mathrm{~mL} / \mathrm{kg}(1 \\
\mathrm{g} / \mathrm{kg})\end{array}$ & $\begin{array}{l}0.5 \\
\mathrm{~mL} / \mathrm{kg} / \mathrm{min} \\
(6 \mathrm{~g} / \mathrm{kg} / \mathrm{hr})\end{array}$ & \begin{tabular}{|l|} 
ILE \\
vs \\
Pre-treatment+ILE
\end{tabular} & \begin{tabular}{|l} 
Other \\
treatment \\
immediately
\end{tabular} & \begin{tabular}{|l|} 
Cardiac \\
massage (LA \\
groups); \\
Fatty-acid \\
oxidation \\
inhibitor CVT \\
(one group); \\
Phosphate \\
buffered saline \\
(one group) \\
\end{tabular} & \begin{tabular}{|l|} 
Oxygen consumption, \\
membrane potential and \\
calcium-retention capacity \\
(in response to Ca2+ \\
overload)
\end{tabular} & $\begin{array}{l}\text { Survival rate } \\
\text { unclear }\end{array}$ & $\begin{array}{l}\text { Not studied; Fatty-acid } \\
\text { oxidation is required } \\
\text { for successful rescue of } \\
\text { bupivacaine-induced } \\
\text { cardiotoxicity by ILE }\end{array}$ \\
\hline \begin{tabular}{|l}
$\begin{array}{l}\text { Partownavid } 2012 \\
(119) \text { ฉ } \\
\text { (Rats) }\end{array}$ \\
\end{tabular} & $\begin{array}{l}\text { Case series; } \\
\text { resuscitation } \\
\text { model }\end{array}$ & \begin{tabular}{|l|} 
Bupivacaine \\
$(10 \mathrm{mg} / \mathrm{kg})$
\end{tabular} & 2.68 & Asystole & NR & $5 \mathrm{~mL} / \mathrm{kg}$ & $\begin{array}{l}0.5 \\
\mathrm{~mL} / \mathrm{kg} / \mathrm{min}\end{array}$ & \begin{tabular}{|l|} 
ILE \\
vs \\
Pre-treatment+ILE
\end{tabular} & \begin{tabular}{|l} 
Other \\
treatment \\
immediately
\end{tabular} & \begin{tabular}{|l|} 
Pre-treatment \\
with fatty-acid \\
oxidation \\
inhibitor CVT \\
(one group) \\
\end{tabular} & $\begin{array}{l}\mathrm{HR} \text {, ejection fraction and } \\
\text { fractional shortening } \\
\text { measures by ECG at } \\
\text { baseline, } 1,5,10 \mathrm{~min} .\end{array}$ & $\begin{array}{l}\text { Survival rate } \\
\text { unclear }\end{array}$ & $\begin{array}{l}\text { Not studied; ILE rescue } \\
\text { of bupivacaine-induced } \\
\text { cardiotoxicity is } \\
\text { abolished by fatty acid } \\
\text { oxidation Inhibitor } \\
\text { CVT-4325 }\end{array}$ \\
\hline $\begin{array}{l}\text { Yoshimoto, } 2012 \\
\text { (120) } \& \text { (Rats) }\end{array}$ & \begin{tabular}{|l|} 
Case series; \\
compared ILE \\
effect between 2 \\
LA, no control \\
\end{tabular} & \begin{tabular}{|l|} 
Levobupivacaine \\
$(2 \mathrm{mg} / \mathrm{kg} / \mathrm{hr})$ \\
or \\
Ropivacaine \\
$(2 \mathrm{mg} / \mathrm{kg} / \mathrm{hr})$
\end{tabular} & 2.68 & \begin{tabular}{|l|} 
Pulse pressure \\
decrease to zero \\
(0 $\mathrm{mmHg}, \mathrm{MAP}$ \\
$6.8-7.6 \pm 1.3$ \\
$\mathrm{mmHg}, \mathrm{HR} 40-$ \\
$47 \pm 15 \mathrm{bm})$
\end{tabular} & $\begin{array}{l}30 \%, \\
\text { brand NR }\end{array}$ & \begin{tabular}{|l|}
$5 \mathrm{~mL} / \mathrm{kg}$ \\
$(1.5 \mathrm{~g} / \mathrm{kg})$
\end{tabular} & $\begin{array}{l}0.5 \\
\mathrm{~mL} / \mathrm{kg} / \mathrm{min} \\
(9 \mathrm{~g} / \mathrm{kg} / \mathrm{hr})\end{array}$ & \begin{tabular}{|l|} 
Levobupivacaine+ \\
ILE \\
vs \\
Ropivacaine+ILE
\end{tabular} & \begin{tabular}{|l|}
$\begin{array}{l}\text { Other } \\
\text { treatment and } \\
\text { study treatment } \\
\text { immediately } \\
\text { after LA }\end{array}$ \\
\end{tabular} & \begin{tabular}{|l|} 
chest \\
compressions \\
and mechanical \\
ventilation \\
\end{tabular} & 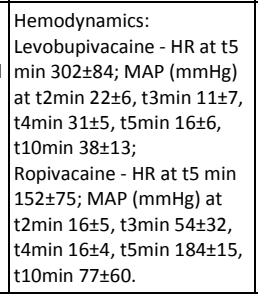 & \begin{tabular}{|l} 
Survival: \\
Levobupi+ILE 6/6, \\
Ropi+ILE 6/6
\end{tabular} & Not studied \\
\hline \begin{tabular}{|l|} 
Case report \\
\end{tabular} & & & & & & & & & & & & & \\
\hline
\end{tabular}


AACT manuscript LA to edit for CTX

\begin{tabular}{|c|c|c|c|c|c|c|c|c|c|c|c|c|c|}
\hline $\begin{array}{l}\text { Reference } \\
\text { (Species) }\end{array}$ & Model & $\begin{array}{l}\text { Local } \\
\text { anesthetic } \\
\text { (dose) }\end{array}$ & $\left.\mid \begin{array}{l}\text { Log } \\
D \\
(129)\end{array}\right)$ & Symptoms & $\begin{array}{l}\text { ILE } \\
\text { used }\end{array}$ & \begin{tabular}{|l|} 
ILE \\
bolus \#
\end{tabular} & \begin{tabular}{|l} 
ILE \\
infusion \\
$\#$
\end{tabular} & Study arms & \begin{tabular}{l|} 
Timing of \\
rescue, \\
time from \\
LA \\
termination
\end{tabular} & $\begin{array}{l}\text { Other } \\
\text { treatments } \\
\text { received * }\end{array}$ & $\begin{array}{l}\text { Parameter } \\
\text { measured }\end{array}$ & Outcome & $\begin{array}{l}\text { Support } \\
\text { therapeutic } \\
\text { effect of ILE } \\
\text { alone }\end{array}$ \\
\hline \begin{tabular}{|l|}
$\begin{array}{l}\text { O'Brien, } 2010(121) \\
\text { (Cat) }\end{array}$ \\
\end{tabular} & Case report & \begin{tabular}{|l|} 
Lidocaine \\
$(20 \mathrm{mg} / \mathrm{kg})$
\end{tabular} & 1.26 & \begin{tabular}{|l|} 
Lethargy, \\
Erratic, poor- \\
quality pulses \\
with severe \\
hypotension, \\
Almost cardiac \\
arrest, \\
Respiratory \\
distress, \\
pulmonary \\
edema \\
\end{tabular} & \begin{tabular}{|l|}
$20 \%$, \\
Liposyn II
\end{tabular} & No & $\begin{array}{l}1.5 \mathrm{~mL} / \mathrm{kg} \\
(0.6 \\
\mathrm{g} / \mathrm{kg} / \mathrm{hr}) \text { for } \\
30 \mathrm{~min}\end{array}$ & NA & \begin{tabular}{|l|} 
Other \\
treatment \\
immediately, \\
ILE treatment at \\
15 min.
\end{tabular} & $\begin{array}{l}\text { lactate ringer } \\
\text { oxygen }\end{array}$ & \begin{tabular}{|l|} 
Hemodynamics: \\
improvement in \\
cardiovascular variables; \\
CNS: Improvement in \\
behavioral variables (more \\
responsive to stimuli, could \\
hold its head up without \\
assistance).
\end{tabular} & Survived & $\begin{array}{l}\text { Yes; } 15 \text { minutes after } \\
\text { initiation of the ILE } \\
\text { emulsion, the cat was } \\
\text { more responsive to } \\
\text { stimuli }\end{array}$ \\
\hline
\end{tabular}


AACT manuscript LA to edit for CTX

\section{Table 4 Summary estimates with associated GRADE ratings for human controlled studies reporting the effect of ILE on LA toxicity*}

\begin{tabular}{|c|c|c|c|c|c|c|c|}
\hline $\begin{array}{c}\text { No of } \\
\text { Studies } \\
\end{array}$ & Population & \multicolumn{2}{|c|}{ Comparison } & \multicolumn{2}{|c|}{ Summary of Finding } & \multicolumn{2}{|c|}{ Quality of Evidence } \\
\hline $\begin{array}{l}N=1 \\
(11)\end{array}$ & $\begin{array}{l}\text { Healthy } \\
\text { volunteers } \\
\text { receiving } \\
\text { lidocaine infusion } \\
\text { followed by either } \\
\text { Ropivacaine or } \\
\text { Levobupivacaine } \\
\text { in continued } \\
\text { infusion (8 } \\
\mathrm{mg} / \mathrm{min}) \\
\end{array}$ & $\begin{array}{l}20 \% \text { Intralipid } \\
120 \text { mL bolus } 2 \\
\text { min after the } \\
\text { start of LA } \\
\text { infusion ( } \mathrm{n}=16 \\
\text { for each LA } \\
\text { infusion) }\end{array}$ & $\begin{array}{c}\text { Saline } \\
\text { (n=16 for each } \\
\text { LA infusion) }\end{array}$ & $\begin{array}{l}\text { Prolong QRS was } \\
\text { present at the end of } \\
\text { the LA infusion } \\
\text { when compared } \\
\text { with baseline, but no } \\
\text { difference in PR, QTc } \\
\text { or QRS duration } \\
\text { between groups (P } \\
=0.68)\end{array}$ & $\begin{array}{l}\text { No difference in } \\
\text { EKG between } \\
\text { groups. }\end{array}$ & $\begin{array}{l}\text { RCT cross-over; } \\
\text { Downgrade: Indirectness } \\
\text { due to surrogate marker } \\
(-1) \text { and subclinical } \\
\text { toxicity design }(-1) \\
\text { Imprecision due to small } \\
\text { sample size }(-1)\end{array}$ & Very low \\
\hline \multicolumn{8}{|c|}{ Neurotoxicity } \\
\hline $\begin{array}{l}N=1 \\
(11)\end{array}$ & $\begin{array}{c}\text { Healthy } \\
\text { volunteers } \\
\text { receiving } \\
\text { lidocaine infusion } \\
\text { followed by either } \\
\text { Ropivacaine or } \\
\text { Levobupivacaine } \\
\text { in continued } \\
\text { infusion (8 } \\
\mathrm{mg} / \mathrm{min}) \\
\end{array}$ & $\begin{array}{l}20 \% \text { Intralipid } \\
120 \text { mL bolus } 2 \\
\text { min after the } \\
\text { start of LA } \\
\text { infusion ( } \mathrm{n}=16 \\
\text { for each LA } \\
\text { infusion) }\end{array}$ & $\begin{array}{c}\text { Saline } \\
\text { (n=16 for each } \\
\text { LA infusion) }\end{array}$ & $\begin{array}{c}\text { MD Ropivacaine = - } \\
6.0(-24.7 ;+12.7) . \\
\text { MD Levobupivacaine } \\
=-11.4(-32.4 ; 9.6) . \\
\text { No EEG } \\
\text { abnormalities } \\
\text { observed. }\end{array}$ & $\begin{array}{l}\text { No difference in } \\
\text { LA doses needed } \\
\text { to reach } \\
\text { neurotoxicity or in } \\
\text { EEG changes } \\
\text { between groups. }\end{array}$ & $\begin{array}{c}\text { RCT cross-over; } \\
\text { Downgrade: } \\
\text { Indirectness due to } \\
\text { surrogate marker (-1) } \\
\text { and subclinical toxicity } \\
\text { design }(-1) \text {, Imprecision } \\
\text { due to small sample size } \\
(-1)\end{array}$ & Very low \\
\hline
\end{tabular}

*Summary estimate is expressed in difference between the "group intervention - group comparator". Either a risk difference (RD), a mean difference (MD) or weighted mean difference (WMD) was reported.

**Quality assessment according to the GRADE methodology. Of note, since no controlled studies were pooled together to answer a specific clinical question, inconsistency and publication bias were not evaluable. 
AACT manuscript LA to edit for CTX

\author{
References
}

1. Rosenblatt MA, Abel M, Fischer GW, Itzkovich CJ, Eisenkraft JB. Successful use of a $20 \%$ lipid emulsion to resuscitate a patient after a presumed bupivacaine-related cardiac arrest. Anesthesiology. 2006;105:217-218.

2. AAGBI. AAGBI Safety Guideline - Management of Severe Local Anaesthetic Toxicity2010. [cited 2015 May 20th]. Available at:

http://www.aagbi.org/sites/default/files/la toxicity 2010 0.pdf

3. American College of Medical T. ACMT position statement: interim guidance for the use of lipid resuscitation therapy. J Med Toxicol. 2011;7:81-82.

4. $\quad$ ASRA. Checklist for treatment of local anesthetic systemic toxicity2011. [cited 2015 May 20th]. Available at: https://www.asra.com/advisory-guidelines/article/3/checklistfor-treatment-of-local-anesthetic-systemic-toxicity

5. Cao D, Heard K, Foran M, Koyfman A. Intravenous lipid emulsion in the emergency department: a systematic review of recent literature. The Journal of emergency medicine. 2015;48:387-397.

6. Cave G, Harvey M, Graudins A. Intravenous lipid emulsion as antidote: a summary of published human experience. Emerg Med Australas. 2011;23:123-141.

7. $\quad$ Corman SL, Skledar SJ. Use of lipid emulsion to reverse local anesthetic-induced toxicity. Ann Pharmacother. 2007;41:1873-1877.

8. Jamaty C, Bailey B, Larocque A, Notebaert E, Sanogo K, Chauny JM. Lipid emulsions in the treatment of acute poisoning: a systematic review of human and animal studies. Clin Toxicol (Phila). 2010;48:1-27.

9. Gosselin S, Morris M, Nesbitt-Miller A, Hoffman RS, Hayes B, Turgeon AF, et al. Methodology for AACT Evidence-based recommendations on the use of Lipid Emulsion Therapy in poisoning. Clin Toxicol (Phila). 2015;53:557-564.

10. Dureau P, Charbit B, Benhamou D, Mazoit JX. La toxicité systémique des anesthésiques locaux peut-elle être prévenue par l'Intralipide $\AA$ ? Une étude pharmacocinétique [Abstract]. Ann Fr Anesth Reanim. 2014;33, Supplement 2:A7.

11. Dureau P, Mazoit JX, Navarro V, Benhamou D, Charbit B. La toxicité systémique des anesthésiques locaux peut-elle être prévenue par l'Intralipide® ? [Abstract]. Ann Fr Anesth Reanim. 2014;33, Supplement 2:A7-A8.

12. Admani B, Essajee F. Successful resuscitation of a three month old child with intralipid infusion, presumed to have bupivacaine induced seizures and cardiovascular complications: case report. East Afr Med J. 2010;87:354-356.

13. Al-Alami AA. Successful treatment of early ropivacaine toxicity with intralipid in a patient with attention deficit hyperactivity disorder. Middle East J Anesthesiol. 2011;21:427-429.

14. Aveline C, Cognet F, Bonnet F. Ineffectiveness of intralipid infusion for central nervous toxicity following ultrasound-guided sciatic nerve block with lidocaine-ropivacaine 


\section{AACT manuscript LA to edit for CTX}

solution: Interaction between carbamazepine, local anaesthetic and intralipid? Eur J Anaesthesiol. 2010;27:1070-1072.

15. Bazerbachi F, Rank K, Chan A. Intravenous lipid rescue and ropivacaine systemic toxicity. J Anesth. 2014;28:139.

16. Bilotta F, Titi L, Rosa G. Local anesthetic-induced complete atrioventricular block during awake craniotomy. Journal of neurosurgical anesthesiology. 2012;24:238.

17. Buckenmaier ICC, Capacchione J, Mielke AR, Bina S, Shields C, Kwon KH, et al. The effect of lipid emulsion infusion on postmortem ropivacaine concentrations in swine: Endeavoring to comprehend a soldier's death. Anesth Analg. 2012;114:894-900.

18. Calenda E, Dinescu SA. Failure of lipid emulsion to reverse neurotoxicity after an ultrasound-guided axillary block with ropivacaine and mepivacaine. J Anesth. 2009;23:472473.

19. Cave G, Harvey M, Willers J, Uncles D, Meek T, Picard J, et al. LIPAEMIC report: results of clinical use of intravenous lipid emulsion in drug toxicity reported to an online lipid registry. J Med Toxicol. 2014;10:133-142.

20. Charbonneau H, Marcou TAP, Mazoit JX, Zetlaoui PJ, Benhamou D. Early use of lipid emulsion to treat incipient mepivacaine intoxication. Reg Anesth Pain Med. 2009;34:277278.

21. Contargyris C, Bourgoin A, Guilhaumou R, Leone M, Martin C. Local anesthetics toxicity during a post caesarian transverse abdominis plane block. Prat Anesth Reanim. 2012;16:308-310.

22. Cordell CL, Schubkegel T, Light TR, Ahmad F. Lipid Infusion Rescue for BupivacaineInduced Cardiac Arrest After Axillary Block. J Hand Surg. 2010;35:144-146.

23. Dacosta WP. Subcutaneous bupivacaine intoxication managed with lipid emulsion [Abstract]. Anestesia en Mexico. 2009;21:143-144.

24. Diaz J, Bernasinski M, Malinovsky JM. Reversal of neurologic symptoms related to lidocaine toxicity with a lipid emulsion administration. [French]. Ann Fr Anesth Reanim. 2012;31:647.

25. Dix SK, Rosner GF, Nayar M, Harris JJ, Guglin ME, Winterfield JR, et al. Intractable cardiac arrest due to lidocaine toxicity successfully resuscitated with lipid emulsion. Crit Care Med. 2011;39:872-874.

26. Egan T. Seizure post brachial plexus block in patient with remote history of seizures with non toxic dose of local anaesthetic [Abstract]. Reg Anesth Pain Med. 2013;38.

27. Espinet AJ, Emmerton MT. The successful use of intralipid for treatment of local anesthetic-induced central nervous system toxicity: Some considerations for administration of intralipid in an emergency. The Clinical journal of pain. 2009;25:808-809.

28. Etesse B, Letouzey V, Roger C, Lefauconnier A, Ripart J. Epidural analgesia is not the only cause of peripartum central neurologic symptoms. Report of one case of posterior reversible encephalopathy syndrome. [French]. Ann Fr Anesth Reanim. 2011;30:57-60. 


\section{AACT manuscript LA to edit for CTX}

29. Fenten MGE, Rohrbach A, Wymenga AB, Stienstra R. Systemic local anesthetic toxicity after local infiltration analgesia following a polyethylene tibial insert exchange: A case report. Reg Anesth Pain Med. 2014;39:264-265.

30. Foxall G, McCahon R, Lamb J, Hardman JG, Bedforth NM. Levobupivacaine-induced seizures and cardiovascular collapse treated with Intralipid®. Anaesthesia. 2007;62:516518.

31. French LK, Kusin S, Hendrickson RG. Pediatric lidocaine toxicity following intraosseous injection: A case series. Clin Toxicol (Phila). 2012;50:329-330.

32. Fuzaylov G, Ying B, Tang Y, Sethna NF. Successful resuscitation after inadvertent intravenous injection of bupivacaine in an adolescent. Paediatr Anaesth. 2010;20:958-959.

33. Gallagher C, Tan JM, Foster CG. Lipid rescue for bupivacaine toxicity during cardiovascular procedures. Heart Int. 2010;5:20-21.

34. Gnaho A, Eyrieux S, Gentili M. Cardiac arrest during an ultrasound-guided sciatic nerve block combined with nerve stimulation. Reg Anesth Pain Med. 2009;34:278.

35. Goyal R, Shukla RN, Kumar G, Tandon M. Supraventricular tachycardia after an intercostal nerve block with bupivacaine treated with $10 \%$ intralipid. J Anaesthesiol Clin Pharmacol. 2011;27:564-565.

36. Grenc D, Sarc L, Knafelj R, Vengust R, Diallo-Renko D. Successful Lipid Emulsion Treatment for Generalized Seizures and Cardiac Arrest Following Epidural Lidocaine Administration. Clin Toxicol (Phila). 2011;49:268.

37. Hartley RA, Foster PN, Moore JA. Local anaesthetic toxicity with continuous local anaesthetic infusion following hepatic resection. Eur J Anaesthesiol. 2012;29:455-456.

38. Harvey M, Cave G, Chanwai G, Nicholson T. Successful resuscitation from bupivacaine-induced cardiovascular collapse with intravenous lipid emulsion following femoral nerve block in an emergency department. EMA. 2011;23:209-214.

39. Heavner J, Heavner M. Cardiac arrest following pleurodesis: Lipid rescue for lidocaine overdose [Abstract]. Chest. 2012;142:291A.

40. Hurley WT, Hanlon P. Lipid emulsion as an antidote at the Washington Poison Center; Use in Carbamazepine, Flecanide, Hydroxychloroquine, Bupivacaine, and Bupropion. Clin Toxicol (Phila). 2009;47:729-730.

41. Jensen K, Borglum J. High volume nerve blocks and systemic toxicity of local anaesthetics. A case report [Abstract]. Reg Anesth Pain Med. 2011;36:E213.

42. Landy C, Gagnon N, Boulland P, Raynaud L, Plancade D. Seizures associated with local anaesthetic intoxication. Br J Anaesth. 2012;109:463-364.

43. Landy C, Schaeffer E, Raynaud L, Favier JC, Plancade D. Convulsions after normal dose of lidocaine: A probable drug interaction. Br J Anaesth. 2012;108:701.

44. Lange DB, Schwartz D, DaRoza G, Gair R. Use of intravenous lipid emulsion to reverse central nervous system toxicity of an iatrogenic local anesthetic overdose in a patient on peritoneal dialysis.[Erratum appears in Ann Pharmacother 2013;47:139]. Ann Pharmacother. 2012;46:e37. 
AACT manuscript LA to edit for CTX

45. Larson A, Stidham T, Banerji S, Kaufman J. Seizures and methemoglobinemia in an infant after excessive EMLA application. Pediatr Emerg Care. 2013;29:377-379.

46. Levine M, Skolnik AB, Ruha AM, Bosak A, Menke N, Pizon AF. Complications following antidotal use of intravenous lipid emulsion therapy. J Med Toxicol. 2014;10:1014.

47. Li J, Wardhan R. Local anesthetic systemic toxicity (LAST) in an ESKD patient upon receiving an axillary brachial plexus block along with blocks of the musculocutaneous \& intercostobrachial nerves [Abstract]. Reg Anesth Pain Med. 2013;38:A218.

48. Lin EP, Aronson LA. Successful resuscitation of bupivacaine-induced cardiotoxicity in a neonate. Paediatr Anaesth. 2010;20:955-957.

49. Litz RJ, Popp M, Stehr SN, Koch T. Successful resuscitation of a patient with ropivacaine-induced asystole after axillary plexus block using lipid infusion. Anaesthesia. 2006;61:800-801.

50. Litz RJ, Roessel T, Heller AR, Stehr SN. Reversal of central nervous system and cardiac toxicity after local anesthetic intoxication by lipid emulsion injection. Anesth Analg. 2008;106:1575-1577.

51. Liu SK, Ou Yang HH, Chen KB, Poon KS, Chang JH. Successful treatment of highly potential local anesthetic systemic toxicity by "staged prevention strategy" with intravenous lipid emulsion [Abstract]. Eur J Anaesth. 2012;29:129.

52. Ludot H, Tharin JY, Belouadah M, Mazoit JX, Malinovsky JM. Successful resuscitation after ropivacaine and lidocaine-induced ventricular arrhythmia following posterior lumbar plexus block in a child. Anesth Analg. 2008;106:1572-1574.

53. Markowitz S, Neal JM. Immediate lipid emulsion therapy in the successful treatment of bupivacaine systemic toxicity [Abstract]. Reg Anesth Pain Med. 2009;34:276.

54. Marraffa JM, Stork CM. Delayed cardiovascular toxicity after inadvertent subcutaneous injection of bupivacaine successfully treated with intravenous fat emulsion. Clin Toxicol (Phila). 2013;51:652-653.

55. Marwick PC, Levin AI, Coetzee AR. Recurrence of cardiotoxicity after lipid rescue from bupivacaine-induced cardiac arrest. Anesth Analg. 2009;108:1344-1346.

56. Mazoit JX. Cardiac arrest and local anaesthetics. [French]. Presse Med. 2013;42:280286.

57. McCutchen T, Gerancher JC. Early Intralipid Therapy May Have Prevented Bupivacaine-Associated Cardiac Arrest. Reg Anesth Pain Med. 2008;33:178-180.

58. Mizutani K, Oda Y, Sato H. Successful treatment of ropivacaine-induced central nervous system toxicity by use of lipid emulsion: Effect on total and unbound plasma fractions. J Anesth. 2011;25:442-445.

59. Nguyen VH, White JL. Further support for the early administration of lipid emulsion in the treatment of ropivacaine-induced central nervous system toxicity. J Anesth.

2012;26:479-480. 


\section{AACT manuscript LA to edit for CTX}

60. Ogugua CS, Savel RH, Keene A, Egesi A, Eisen L. Prevention of catastrophic outcomes in systemic bupivacaine toxicity: Early recognition and aggressive management [Abstract]. Chest. 2009;136:38S-e-39S.

61. Reddy JG, Lahm T. Intravenous lipid emulsion rescue for severe central nervous system local anesthetic toxicity [Abstract]. Chest. 2010;138:76A.

62. Sakai T, Manabe W, Kamitani T, Takeyama E, Nakano S. [Ropivacaine-induced lateonset systemic toxicity after transversus abdominis plane block under general anesthesia: successful reversal with 20\% lipid emulsion]. Masui. 2010;59:1502-1505.

63. Schaeffer E, Rayaud L, Landy C, Boulland P, Favier JC. Local anaesthetics intoxication, during ultrasound-guided axillary plexus block, treated by Intralipide $囚$. Ann Fr Anesth Reanim. 2010;29:929-930.

64. Schellhammer F, Milde A. Lipid rescue therapy of toxic effects from local anesthetics in interventional radiology. RöFo. 2011;183:73-74.

65. Scherrer V, Compere V, Loisel C, Dureuil B. Cardiac arrest from local anesthetic toxicity after a field block and transversus abdominis plane block: A consequence of miscommunication between the anesthesiologist and surgeon. A A Case Rep. 2013;1:75-76. 66. Schwarzkopf P, Ruffert H, Pracht K. Psoas compartment block: How to limit systemic side effects? [Abstract]. Reg Anesth Pain Med. 2011;36:E261.

67. Shah S, Gopalakrishnan S, Apuya J, Shah S, Martin T. Use of Intralipid in an infant with impending cardiovascular collapse due to local anesthetic toxicity. J Anesth. 2009;23:439-441.

68. Shenoy U, Paul J, Antony D. Lipid resuscitation in pediatric patients-need for caution? Paediatr Anaesth. 2014;24:332-334.

69. Shih YH, Chen CH, Wang YM, Liu K. Successful reversal of bupivacaine and lidocaineinduced severe junctional bradycardia by lipid emulsion following infraclavicular brachial plexus block in a uremic patient. Acta Anaesthesiol Taiwan. 2011;49:72-74.

70. Smith HM, Jacob AK, Segura LG, Dilger JA, Torsher LC. Simulation education in anesthesia training: a case report of successful resuscitation of bupivacaine-induced cardiac arrest linked to recent simulation training. Anesth Analg. 2008;106:1581-1584.

71. Sonsino DH, Fischler M. Immediate intravenous lipid infusion in the successful resuscitation of ropivacaine-induced cardiac arrest after infraclavicular brachial plexus block. Reg Anesth Pain Med. 2009;34:276-277.

72. Sorrenti D, Peverini M, Casalena S, Galletti M. Late onset local anaesthetic systemic toxicity (last) after ENS guided axillary brachial plexus block with mepivacaine 1.5\%: Successful reversal with early 20\% lipid emulsion therapy [Abstract]. Reg Anesth Pain Med. 2014;39:e260.

73. Spence AG. Lipid reversal of central nervous system symptoms of bupivacaine toxicity. Anesthesiology. 2007;107:516-517.

74. Sturini E, Saporito A, Tomasetti R, Anselmi L. Mepivacaine neurologic toxicity after an erroneous administration for intravenous regional anesthesia treated with lipid 
AACT manuscript LA to edit for CTX

emulsion: New solutions for old mistakes [Abstract]. Reg Anesth Pain Med. 2010;35:E179E180.

75. Süzer MA, Özhan MO, Eşkin MB, Atik B, Çaparlar C. Local anesthetic toxicity managed successfully with lipid infusion (case report). Türk Anest Rean Der Dergisi. 2011;39:159163.

76. Ter Horst MT, Tjiang GCH, Luitwieler RL, Van Velzen C, Stolker RJ, De Quelerij M. Antidote for intoxication due to local anesthetics: New application of fat emulsion for intravenous administration. Ned Tijdschr Geneeskd. 2010;154:1822-1825.

77. Varela H, Bums SM. Use of lipid emulsions for treatment of local anesthetic toxicity: a case report. AANA J. 2010;78:359-364.

78. Warren JA, Thoma RB, Georgescu A, Shah SJ. Intravenous lipid infusion in the successful resuscitation of local anesthetic-induced cardiovascular collapse after supraclavicular brachial plexus block. Anesth Analg. 2008;106:1578-1580.

79. Whiteman DM, Kushins SI. Successful Resuscitation With Intralipid After Marcaine Overdose. Aesthetic surgery journal / the American Society for Aesthetic Plastic surgery. 2014;34:738-740.

80. Whiteside J. Reversal of local anaesthetic induced CNS toxicity with lipid emulsion Anaesthesia. 2008;63:203-204.

81. Widfeldt N, Kolmodin L. CNS symptoms of ropivacaine in bloodless field disappeared with Intralipid. [Norwegian]. Lakartidningen. 2014;111:742-743.

82. Wong GK, Joo DT, McDonnell C. Lipid resuscitation in a carnitine deficient child following intravascular migration of an epidural catheter. Anaesthesia. 2010;65:192-195.

83. Zhurda T, Muzha D, Caushi GJ, Bajaktari M, Kerci M. Usefulness of lipidic solution for the treatment of systemic toxicity related to bupivacaine injected in a sub-coracoid brachial plexus block. [French]. Ann Fr Anesth Reanim. 2010;29:592-593.

84. Zimmer C, Piepenbrink K, Riest G, Peters J. Cardiotoxic and neurotoxic effects after accidental intravascular bupivacaine administration. Therapy with lidocaine propofol and lipid emulsion. [German]. Anaesthesist. 2007;56:449-453.

85. Bonfim MR, De Simone Melo M, Dreyer E, Borsoi LFA, de Oliveira TG, Udelsmann A. Lipid Therapy with Two Agents in Ropivacaine-Induced Toxicity: Experimental Study in Swine. Rev Bras Anestesiol. 2012;62:685-695.

86. Bushey BA, Auld VH, Volk JE, Vacchiano CA. Combined Lipid Emulsion and ACLS Resuscitation Following Bupivacaine- and Hypoxia-lnduced Cardiovascular Collapse in Unanesthetized Swine. AANA Journal. 2011;79:129-138.

87. Candela D, Louart G, Bousquet PJ, Muller L, Nguyen M, Boyer JC, et al. Reversal of bupivacaine-induced cardiac electrophysiologic changes by two lipid emulsions in anesthetized and mechanically ventilated piglets. Anesth Analg. 2010;110:1473-1479. 88. De Queiroz M, Rhondali O, Leveneur 0, Musard H, Chassard D. Resuscitation with lipid or with epinephrine in levobupivacaine-induced cardiac toxicity in piglets. $\mathrm{Br} \mathrm{J}$ Anaesth. 2012;108:ii292-ii293. 


\section{AACT manuscript LA to edit for CTX}

89. de Queiroz Siqueira M, Chassard D, Musard H, Heilporn A, Cejka JC, Leveneur O, et al. Resuscitation with lipid, epinephrine, or both in levobupivacaine-induced cardiac toxicity in newborn piglets. Br J Anaesth. 2014;112:729-734.

90. de Simone Melo M, Bonfm MR, Dreyer E, Bassanezi BSB, Udelsmann A.

Hemodynamic changes in lipid emulsion therapy (SMOFlipid) for bupivacaine toxicity in swines. Acta Cir Bras. 2012;27:318-324.

91. Di Gregorio G, Kessler C, Weinberg G. Lipid emulsion in treatment of sodium channel blocker overdose. Ann Emerg Med. 2008;51:489.

92. Di Gregorio G, Schwartz D, Ripper R, Kelly K, Feinstein DL, Minshall RD, et al. Lipid emulsion is superior to vasopressin in a rodent model of resuscitation from toxin-induced cardiac arrest.[Erratum appears in Crit Care Med 2009;37:2329]. Crit Care Med. 2009;37:993-999.

93. Fettiplace MR, Akpa BS, Ripper R, Zider B, Lang J, Rubinstein I, et al. Resuscitation with lipid emulsion: dose-dependent recovery from cardiac pharmacotoxicity requires a cardiotonic effect. Anesthesiology. 2014;120:915-925.

94. Fettiplace MR, Ripper R, Lis K, Feinstein DL, Rubinstein I, Weinberg G. Intraosseous lipid emulsion: an effective alternative to IV delivery in emergency situations. Crit Care Med. 2014;42:e157-e160.

95. Gokahmetoglu G, Aksu R, Bicer C, Darcin K, Ugur F. The effect of levosimendan combined with $20 \%$ lipid emulsion treatment on survival from bupivacaine induced toxicity in experiment. Bratisl Lek Listy. 2014;115:275-279.

96. Hicks SD, Salcido DD, Logue ES, Suffoletto BP, Empey PE, Poloyac SM, et al. Lipid emulsion combined with epinephrine and vasopressin does not improve survival in a swine model of bupivacaine-induced cardiac arrest. Anesthesiology. 2009;111:138-146.

97. Hiller DB, Gregorio GD, Ripper R, Kelly K, Massad M, Edelman L, et al. Epinephrine impairs lipid resuscitation from bupivacaine overdose: A threshold effect. Anesthesiology. 2009;111:498-505.

98. Karci A, Aykac Ibisoglu A, Oransay K, Olguner G, Elar Z. The effects of lipid treatment on levobupivacaine induced cardiotoxicity in rats [Abstract]. Eur J Anaesthesiol. 2009;26:108.

99. Karcioğlu M, Tuzcu K, Sefil F, Davarci I, Aydin S, Sari A, et al. Efficacy of resuscitation with intralipid in a levobupivacaine-induced cardiac arrest model. Turk J Med Sci. 2014;44:330-336.

100. Li Z, Xia Y, Dong X, Chen H, Xia F, Wang X, et al. Lipid resuscitation of bupivacaine toxicity: Long-chain triglyceride emulsion provides benefits over long-and medium-chain triglyceride emulsion. Anesthesiology. 2011;115:1219-1228.

101. Litonius ES, Niiya T, Neuvonen PJ, Rosenberg PH. Intravenous lipid emulsion only minimally influences bupivacaine and mepivacaine distribution in plasma and does not enhance recovery from intoxication in pigs. Anesth Analg. 2012;114:901-906. 


\section{AACT manuscript LA to edit for CTX}

102. Mauch J, Jurado OM, Spielmann N, Bettschart-Wolfensberger R, Weiss M. Resuscitation strategies from bupivacaine-induced cardiac arrest. Paediatr Anaesth. 2012;22:124-129.

103. Mauch J, Martin Jurado O, Spielmann N, Bettschart-Wolfensberger R, Weiss M. Comparison of epinephrine vs lipid rescue to treat severe local anesthetic toxicity - An experimental study in piglets. Paediatr Anaesth. 2011;21:1103-1108.

104. Mauch JY, Martin-Jurado O, Spielmann N, Bettschart-Wolfensberger R, Weiss M. Comparison of four different rescue regimens to treat cardiac arrest due to bupivacaine intoxication - An experimental study in neonatal pig [Abstract]. Eur J Anaesthesiol. 2011;28:118.

105. Mayr VD, Mitterschiffthaler L, Neurauter A, Gritsch C, Wenzel V, Muller T, et al. A comparison of the combination of epinephrine and vasopressin with lipid emulsion in a porcine model of asphyxial cardiac arrest after intravenous injection of bupivacaine. Anesth Analg. 2008;106:1566-1571.

106. Shi K, Xia Y, Wang Q, Wu Y, Dong X, Chen C, et al. The effect of lipid emulsion on pharmacokinetics and tissue distribution of bupivacaine in rats. Anesth Analg. 2013;116:804-809.

107. Wat CY, Yuen MK, Li RWS, Leung GPH, Ng KFJ. Lipid emulsion infusion vs standard cardiac resuscitation in management of ropivacaine cardiac toxicity in pigs. Anaesth Intensive Care. 2009;37:661-662.

108. Weinberg G, Paisanthasan C, Feinstein D, Hoffman W. The effect of bupivacaine on myocardial tissue hypoxia and acidosis during ventricular fibrillation. Anesth Analg. 2004;98:790-795.

109. Weinberg G, Ripper R, Feinstein DL, Hoffman W. Lipid emulsion infusion rescues dogs from bupivacaine-induced cardiac toxicity. Reg Anesth Pain Med. 2003;28:198-202. 110. Weinberg GL, Di Gregorio G, Ripper R, Kelly K, Massad M, Edelman L, et al. Resuscitation with lipid versus epinephrine in a rat model of bupivacaine overdose. Anesthesiology. 2008;108:907-913.

111. Yan J, Shen Y, Li B, Hu Z, Ma Z. Association of sustained cardiovascular recovery with epinephrine in the delayed lipid-based resuscitation from cardiac arrest induced by bupivacaine overdose in rats. Br J Anaesthesia. 2012;108:857-863.

112. Yoshimoto M, Horiguchi T, Nishikawa T. Efficacy of lipid resuscitation in cardiac arrest induced by bupivacaine-glucose mixture in rats. Eur J Anaesthesiol. 2014;31:210. 113. Callejo D, Sevilla R, Gonzalez J, Quintela O, De Diego C, Zaballos M. Analysis of the temporal regression of the QRS widening induced by bupivacaine after intralipid administration: Study in an experimental porcine model. Eur J Anaesthesiol. 2014;31:65. 114. Cave G, Harvey M, Prince G, Lahner D, Desmet J. Effect of hypertonic saline on electrocardiography QRS duration in rabbit model of bupivacaine toxicity resuscitated by intravenous lipid. Anaesthesia. 2010;65:792-798. 


\section{AACT manuscript LA to edit for CTX}

115. Weinberg GL, VadeBoncouer T, Ramaraju GA, Garcia-Amaro MF, Cwik MJ. Pretreatment or resuscitation with a lipid infusion shifts the dose - Response to bupivacaine-induced asystole in rats. Anesthesiology. 1998;88:1071-1075. 116. Harvey M, Cave G, Prince G, Lahner D. Epinephrine injection in lipid-based resuscitation from bupivacaine-induced cardiac arrest: transient circulatory return in rabbits. Anesth Analg. 2010;111:791-796.

117. Partownavid P, Umar S, Li J, Rahman S, Eghbali M. Fatty-acid oxidation and calcium homeostasis are involved in the rescue of bupivacaine-induced cardiotoxicity by lipid emulsion in rats. Crit Care Med. 2012;40:2431-2437.

118. Partownavid P, Umar S, Rahman S, Eghbali M. Intralipid Rescue of Bupivacaineinduced Cardiotoxicity was Abolished by Fatty Acid Oxidation Inhibitor CVT-4325 [Abstract]. Circulation. 2010;122:no page.

119. Partownavid P, Umar S, Rahman S, Sharma S, Eghbali M. Intralipid fails to rescue bupivacaine-induced cardiotoxicity in the presence of the opioid antagonist naloxone [Abstract]. Circulation. 2012;126.

120. Yoshimoto M, Horiguchi T, Nishikawa T, Tobe Y. Comparative effects of lipid emulsion on the recovery from levobupivacaine-induced or from ropivacaine-induced cardiac arrest in rats. Br J Anaesthesia. 2012;108:ii105-ii106.

121. O'Brien TQ, Clark-Price SC, Evans EE, Di Fazio R, McMichael MA. Infusion of a lipid emulsion to treat lidocaine intoxication in a cat. J Am Vet Med Ass. 2010;237:1455-1458. 122. Balshem H, Helfand M, Schunemann HJ, Oxman AD, Kunz R, Brozek J, et al. GRADE guidelines: 3. Rating the quality of evidence. Journal of clinical epidemiology. 2011;64:401406.

123. Guyatt GH, Oxman AD, Kunz R, Brozek J, Alonso-Coello P, Rind D, et al. GRADE guidelines 6 . Rating the quality of evidence--imprecision. Journal of clinical epidemiology. 2011;64:1283-1293.

124. Guyatt GH, Oxman AD, Kunz R, Woodcock J, Brozek J, Helfand M, et al. GRADE guidelines: 8. Rating the quality of evidence--indirectness. Journal of clinical epidemiology. 2011;64:1303-1310.

125. Guyatt GH, Oxman AD, Kunz R, Woodcock J, Brozek J, Helfand M, et al. GRADE guidelines: 7. Rating the quality of evidence--inconsistency. Journal of clinical epidemiology. 2011;64:1294-1302.

126. Guyatt GH, Oxman AD, Montori V, Vist G, Kunz R, Brozek J, et al. GRADE guidelines: 5 . Rating the quality of evidence--publication bias. Journal of clinical epidemiology. 2011;64:1277-1282.

127. Guyatt GH, Oxman AD, Sultan S, Glasziou P, Akl EA, Alonso-Coello P, et al. GRADE guidelines: 9. Rating up the quality of evidence. Journal of clinical epidemiology. 2011;64:1311-1316.

128. Guyatt GH, Oxman AD, Vist G, Kunz R, Brozek J, Alonso-Coello P, et al. GRADE guidelines: 4. Rating the quality of evidence--study limitations (risk of bias). Journal of clinical epidemiology. 2011;64:407-415. 
AACT manuscript LA to edit for CTX

129. Chem Spider Search and Share Chemistry. 2015 [cited 2015 July 23rd]; Available at: http://www.chemspider.com. 